\title{
TRACE 3-D Documentation
}

\author{
K. R. Crandall
}

\section{DISCLAIMER}

\begin{abstract}
This report was prepared as an account of work sponsored by an agency of the United States Government. Neither the United States Government nor any agency thereof, nor any of their employecs, makes any warranty, express or implied, or assumes any legal liability or responsibility for the accuracy, completeness, or usefulness of any information, apparatus, product, or process disclosed, or represents that its use would not infringe privately owned rights. Reference herein to any specific commercial product, process, or service by trade name, trademark, manufacturer, or otherwise does not necessarily constitute or imply its endorsement, recommendation, or favoring by the United States Government or any agency thereof. The views and opinions of authors expressed herein do not necessarily state or reflect those of the United States Government or any agency thereof.
\end{abstract}

\section{MASTER}
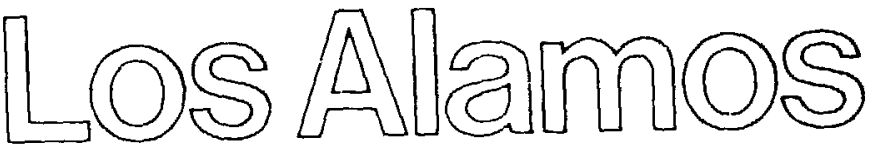


\section{TABLE OF CONTENTS}

AESTRACT

I. INTRODUCTION

I. GENERAL DESCRIPTION

III. COORDINATE SYSTEM AND UNITS . . . . . . . . . . . . . . . . . . . . . . . . 4

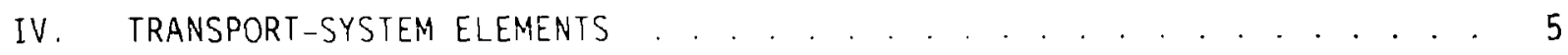

V. DYNAMICS CALCULATIONS . . . . . . . . . . . . . . . . . . . . . . . . . . 8

A. Elements Having Zero Length. . . . . . . . . . . . . . . . . . . . . . 9

B. Elements Having Constant Fields and No Energy Gain . . . . . . . . 9

C. Elements Causing Energy Change or Having Varying fields... . . 9

VI. TRANSFER MATRICES . . . . . . . . . . . . . . . . . . . . . . 9

A. Drift $(\ell)$. . . . . . . . . . . . . . . . . . . . 10

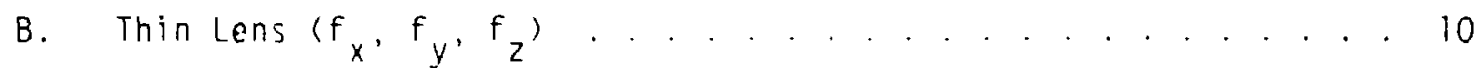

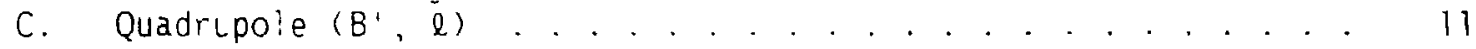

D. $P M Q\left\langle B^{\prime}, \ell, r_{j}, r_{0}\right)$. . . . . . . . . . . . . . . . 11

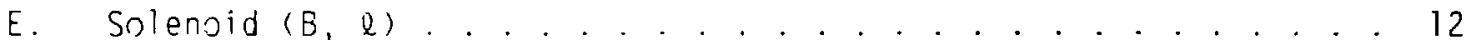

F. Doublet $\left(B^{\prime}, \ell, d\right)$. . . . . . . . . . . . . . . . . 13

G. Triplet $\left(B^{\prime}{ }_{0}, \ell_{0}, d, B^{\prime}{ }_{j}, \ell_{i}\right) . . . . . . . . . . .13$

H. Bending Magnet $(\alpha, \rho, n)$. . . . . . . . . . . . . . . . . 13

I. Edge Angle on Bending Hagnet $\left(B, \rho, g, K_{1}, K_{2}\right) . . . . . . . . .15$

J. Radio-frequency bap $\Sigma_{0} T L, \phi_{S}$, egf, dwf, hl. . . . . . . . . . . 16

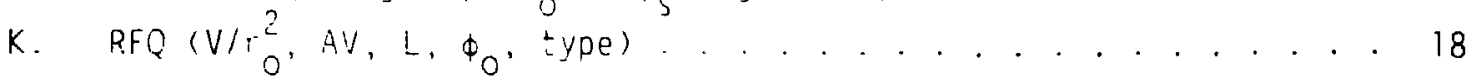

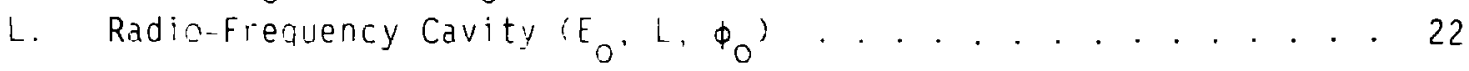

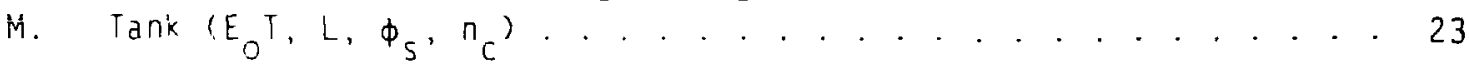

N. Special (l, user-defined parameters) . . . . . . . . . . . . . . . 24

0. Rotation $(A)$. . . . . . . . . . . . . . . . . . . . . . . . . . 24

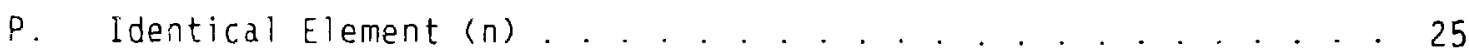

VII. SPACE-ChARGE IMPULSES . . . . . . . . . . . . . . . . . . . . . . . . . . . . . 25

VIII. USER INSTPUCTIONS . . . . . . . . . . . . . . . . . . . . . . . . . . . . . . . . 28 
IX. INPUT VARIABLES . . . . . . . . . . . . . . . . . . . . . . . . . . . . . 29

A. Transport System [NT(NELMAX), A(5,NELMAX), FREQ, PQEXT, and ICHROM] . . . . . . . . . . . . . . . . 30

B. Beam Characteristics [ER, Q, W, XI, BEAMI(6), EMITI(3), $\operatorname{SIGI}(6,6)] \ldots \ldots . \ldots 30$

C. Control Parameters (N1, N2, SMAX, PQSMAX, IBS, NEL1, NEL2) . . 31

D. Matching Parameters [MT, NC, MP(2,6), $\operatorname{MVC}(3,6), \operatorname{BEAMF}(6)$,

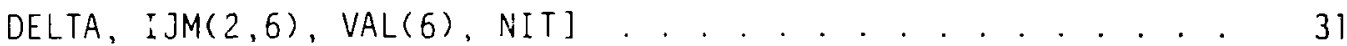

E. Craphics Scales (XM, XPM, YM, DPM, DWM, DPP . . . . . . . 33

F. Print Parameters [NPRIN, IJPRIN(2,20)] . . . . . . . . . . 34

G. Extra Parameters [NXTRA, EXTRA(10)] . . . . . . . . 34

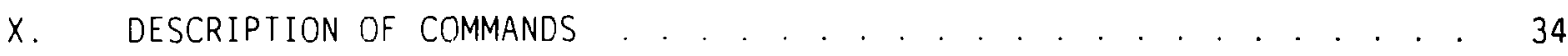

XI. EXAMPLES . . . . . . . . . . . . . . . . . . . . . . . . . . . . . 39

A. Matching Between 400-MHz RFQ and DTL . . . . . . . . . . 39

B. Matching Between 80-MHz RFQ and DTL.......... 51

C. Electron Linac and Transport System............ 55

ACKNOWLEDGMENT . . . . . . . . . . . . . . . . . . . 56

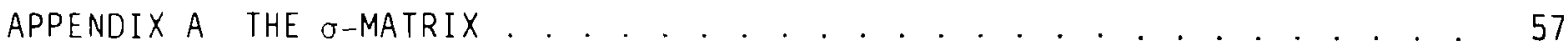

APPENOIX B TWISS PARAMETERS AND THEIR RELATIONSHIPS

TO $\sigma$-MATRIX ELEMENTS . . . . . . . . . . . . . . . . . 61

APPENDIX $C$ MISMATCH FACTOR . . . . . . . . . . . . . . . . . . . . . . . . . . . . 63

APPENDIX D RMS EMITTANCE AND THE EQUIVALENT UNIFORM BEAM . . . . . . . . . 65

APPENDIX E FIELD FORMULAE FOR PERMANENT-MAGNET QUADRUPOLE (PMQ) . . . . . 69

APPENDIX $F$ TRANSVERSE EMITTANCE INCREASE IN AN RF GAP . . . . . . . . . 72

APPENDIX G ELECTROMAGNETIC FIELdS IN CYLINDRICAL CAVITIES ${ }^{13,15} \ldots \ldots$

APPENDIX H COUPLED-CAVITY TANKS . . . . . . . . . . . . . . . . . . . . . . . . . . 78

APPENDIX I TRANSVFRSE EMITTANCE INCREASE FROM CHROMATIC ABERRATIONS . . . 82

APPENDIX $J$ CALCULATIONS OF TRANSVERSE EMITTANCES FOR BEAM-WIDTH

MEASUREMENTS AT THREE LOCATIONS . . . . . . . . . . . . . . 85

REFERENCES . . . . . . . . . . . . . . . . . 86 
TRACE 3-D DOCUMENTATION

\author{
by \\ K. R. Crandall
}

\begin{abstract}
TRACE 3-D is an interactive beam-dynamics program that calculates the envelopes of a bunched beam, including linear space-charge forces, through a user-defined transport system. TRACE 3-D provides an immediate graphics display of the enveiopes and the phase-space ellipses and allows nine types of beam-matching options. This report describes the beamdynamics calculations and gives detailed instruction for using the code. Several examples are described in detail.
\end{abstract}

I. INTRODUCTION

TRACE 3-D is an interactive program that calculates the envelopes of a bunched beam, including linear space-charge forces, through a user-defined transport system. The transport system may consist of the following alements: (1) drift, (2) thin lens, (3) quadrupole, (4) permanent-magnet quadrupole (PMQ), (5) solenoid, (6) doublet, (7) triplet, ( 8 ) bending magnet, (9) edge angle (for bend), (10) rf gap, (11) radio-frequency-quadrupole (RFQ) cel1, (12) rf cavity, (13) coupled-cavity tank, (14) user-defined element, (15) coordinate rotation, and (16) identical element.

The beam is represented by a $6 \times 6$-matrix (introduced by the TRANSPORT program ${ }^{1}$ ) defining a hyperellipsoid in six-dimensional phase space (see App. A). The projection of this hyperellipsoid on any two-dimensional plane is an ellipse that defines the boundary of the beam in that plane. The most useful projection planes are the transverse and longitudinal phase planes in which the eliipses are characterized by the Courant-Snyder, or Twiss, parameters and emittances (see App. B). Using a sequence of matrix transformations, the beam can be "followed" between any two elements. The user can change any parameter and observe the effect on the beam envelopes and on the output-beam ellipses. Also, several matching options are available that determine values for the ellipse parameters or for 
specified transport-system parameters (such as quadrupole gradientr) to meet specified objectives.

As an example in which TRACE 3 a is extremely useful, consider the problem of matching the beam from the exit of an RFQ into the entrance of a drift-tube linac (OTL). This matching is a three-stage process accomplished easily and quickly by TRACE 3-0.

The first stage in the process is to tetermine the natched-beam characteristics at the output of the RFo by tieating the last two RFQ cells as one period in a periodic focusing system. Secause of soace-charge forces that couple the dynamics in the three phase olanes. -h: task involves solving six simultaneous, noni inear equations.

The second jtage is to rind the srect hout beam for the DTL by treating the first two DTL cells ts gne oerig in aeligai focusing system. cone DTL cell consists of the following sequence of elements: quadiupole. drift, rf gap, drift, and quadrupole.)

The final stage is to design a transport system between the RFQ and DTL that will tralisorm the matched beam exiting from the RFQ into the matched beam wanted by the OTL. Quadrupole strengths and drift lengths in the transport system can be automatically :aried by TRACE 3-D in an effort to find a solution to the matching argolem. A satisfactory solution is found when a calculated mismatch factor isee App. C) is less than 0.0001.

TRACE 3-D provides an immediate graphics display, including the beam envelopes and the fhase-space ellipses in the transverse and longitudinal dimensions. This feature, along with the interactive capability, makes the program a learning a id as well as a useful design tool.

TRACE 3-D is an evolution of earlier two-dimensional versions. The original TRACE $^{2}$ was written in 1973 and was developed for use on the controls computer of LiMPF. A modified version ${ }^{3}$ was written for the controls computer of the CERN linac in 1977; in 1979, the CERN version was adapted to the Pion Generator for Medical Irradiations (PIGMI) contiols system at Los Alamos. This yersion was expanded extensively and was adapted for use on the CDC-7600 computer at the central computer facility at Los Alamcs and on the AT-Division VAX-11/750, and is documented in a LOS Alamos report. "

The structure of TRACE $3-0$ allows the code to be easily modified and expanded. Interaction with the progiam, which runs on the CDC-7600 and the CRAY under the LTSS and CTSS operating systems, is through a Tektronix 
4000-series terminal using the Tektronix Plot-10 Terminal Control System. The program can be adapted for any other graphics terminal by replacing the plot-10 subroutine calls by calls to any graphics package that draws lines, plots dots, writes character strings, and allows a mechanism for interaction.

\section{II . GENERAL DESCRIPTION}

The basic assumption of TRACE 3-D is that all forces are linear or can be linearized. If the six coordinates of a particle are known at some location $s_{1}$ along a transport system, then at $s_{2}$ the coordinates can be calculated by a single matrix multiplication. That is,

$\vec{x}\left(s_{2}\right)=R \vec{x}\left(s_{1}\right)$.

where $\vec{x}(s)$ is the $6 \times 1$ column vector of the coordinates at location s, and $R$ is a $6 \times 6$ matrix whose elements depend on the transport elements between $s_{1}$ and $s_{2}$ and on the size of the beam (for computing space-charge forces) in this interval. The R-matrix is referred to as the transfer matrix between locations $s_{1}$ and $s_{2}$. Usually, a transfer matrix representing particle transport over a long distance is determined by a sequence of matrix multiplications of transfer matrices representing particle transport over smaller intervals that comprise the total transport distance.

For the space-charge forces to be linear, one must postulate a beam having a uniform charge distribution in real space. (The electric-field components inside a uniformly charged ellipsoid are given in Sec. VII.) Although real life is rarely so accommodating, it has been shown that for distributions having ellipsoidal symmetry, the evolution of the rms beam envelope depends almost exclusively on the linearized part of the self-forces. 5 Consequently, for calculational purposes the "real beam" may be repiaced by an "equivalent uniform beam" having identical rms properties (sec App. C). The total emittance of the equivalent uniform beam (the beam followed by TRACE 3-D) in each phase plane is five times the rms emittance in that plane, and the displayed beam envelopes are $\sqrt{5}$ times their respective rms values. (Real beams have ill-defined boundaries. In general, one can expect a few percent $(<10 \%)$ of the particles in a real beam to be outside the boundaries displayed by TRACE 3-D.) 
If the transfer matrix between $s_{1}$ and $s_{2}$ is known, and if the beam matrix at $s_{1}$ is known, then the beam matrix at $s_{2}$ is calculated by $\sigma\left(s_{2}\right)=R \sigma\left(s_{1}\right) R^{T}$.

where $R^{\top}$ denotes the transpose of $R$. The dynamics calculations in TRACE 3-D are done by a sequence of transformations as specified above. Starting with an initial o-matrix, a transfer matrix is constructed from the external forces for a small transport interval and a new o-matrix is calculated. The size of the beam is obtained from elements of the o-matrix, and linear space-charge forces are calculated using the beam size. An R-matrix is constructed for the space-charge impulse, and a new o-matrix is calculated. This process is repeated until the beam has been followed through the specified elements.

At the beginning of a calculation, initial phase-space ellipses are displayed at the graphics terminal. At each step, the beam boundaries in real space are displayed, allowing the user to visualize how the bean is behaving. At the end, the final phase-space ellipses are displayed.

\section{COORDINATE SYSTEM AND UNITS}

The internal coordinates and their units are

$$
\vec{x}=\left\{\begin{array}{ll}
x & (m m) \\
x^{\prime} & (m r a d) \\
y & (m m) \\
y^{\prime} & (m r a d) \\
z & (m m) \\
\frac{\Delta p}{p} & (m r a d)
\end{array}\right\},
$$

where $x, y$, and $z$ are horizontal, vertical, and longitudinal displacements from the center of the beam bunch (assumed to be on the equilibrium orbit), $x^{\prime}$ and $y^{\prime}$ are the relative rates at which the particie is moving away from the horizontal and vertical axes, and $\Delta p / p$ is the relativs difference in the particle's longitudinal momentum from the longitudinal momentum of the center of the beam bunch. For input and output, however, $z$ and $\Delta p / p$ are replaced 
by $\Delta \phi$ and $\Delta W$, the phase and energy displacements in degrees and keV. The relationships between these longitudinal coordinates are

$z=-\frac{\beta \lambda}{360} \Delta \phi$,

and

$\frac{\Delta p}{p}=\frac{Y}{Y+1} \frac{\Delta W}{W}$.

wheie $\beta$ and $\gamma$ are the usual relativistic parameters, $\lambda$ is the free-space wavelength of the $r f$, and $W$ is the kinetic energy in MeV at the beam center. Phase and energy coordinates are more normal coordinates for discussion of input/ouput parameters of accelerator-related transport systems than are $z$ and $\Delta p / p$; however, internal calculations are simplified by using the $z$ and $\Delta p / p$ coordinates.

The units of the coordinate system define the units of the transfermatrix elements, which are either dimensionless, $\mathrm{m}$, or $\mathrm{m}^{-1}$ :

$\left[\begin{array}{cccccc}1 & m & 1 & m & 1 & m \\ m^{-1} & 1 & m^{-1} & 1 & m^{-1} & 1 \\ 1 & m & 1 & m & 1 & m \\ m^{-1} & 1 & m^{-1} & 1 & m^{-1} & 1 \\ 1 & m & 1 & m & 1 & m \\ m^{-1} & 1 & m^{-1} & 1 & m^{-1} & 1\end{array}\right]$.

\section{TRANSPORT-SYSTEM ELEMENTS}

Each transport element is defined by a "type code" and by five or fewer parameters, summarized in Table I (parameter definitions follow the table). Unless otherwise specified, all conventions assume a posizive beam, all lengths are in millimeters (mm), all magnetic-field gradients are in teslas/meter $(T / m)$, and all angles are in degrees $\left({ }^{\circ}\right)$. Positive magnetic gradients are focusing in the horizontal plane and defocusing in the vertical plane for positive beams. 
TABLE I

DEFINITION OF TRANSPORT ELEMENTS

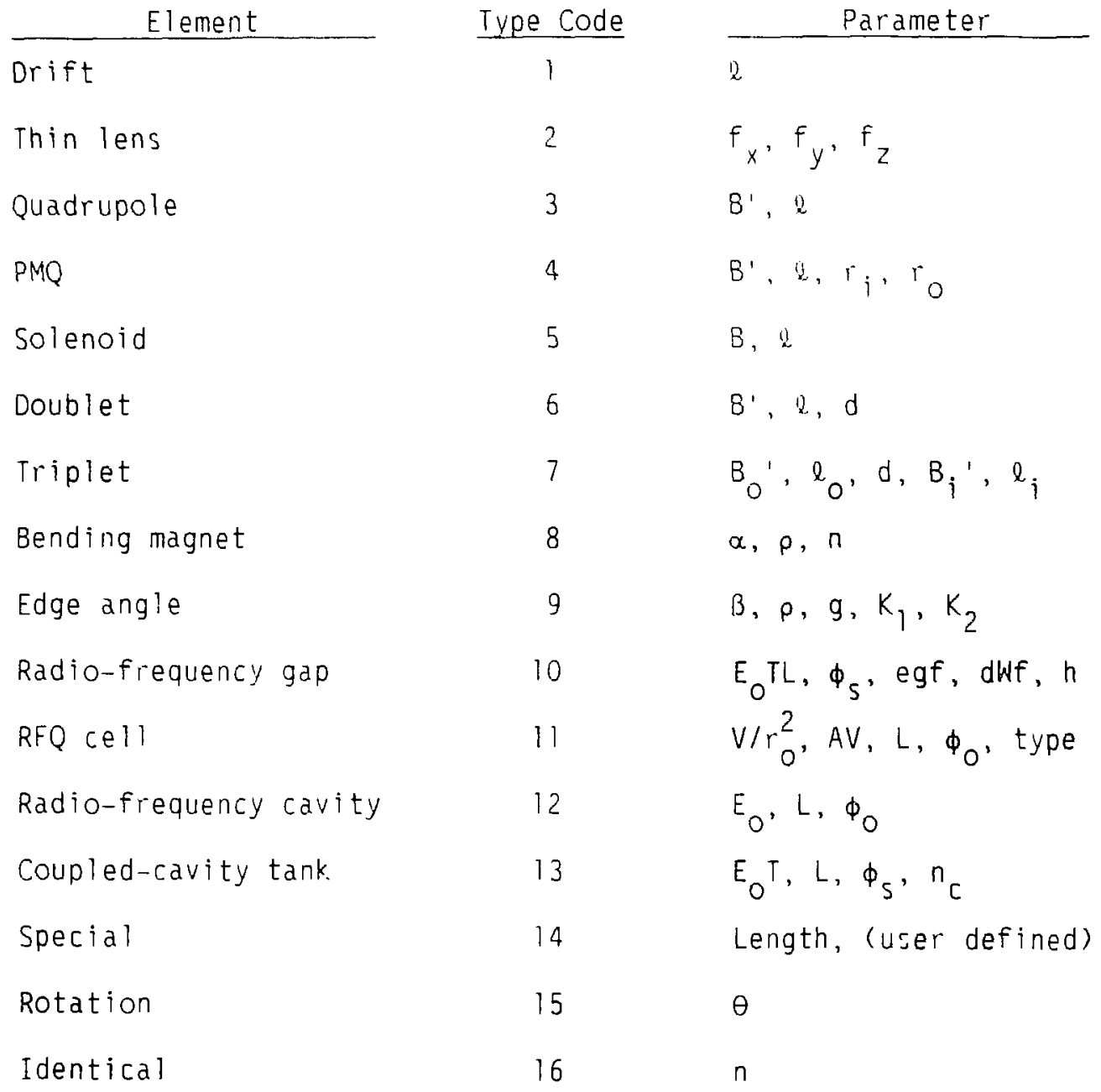

6 
DEFINITION OF PARAMETERS USED IN TABLE I

Element

1. Drift

2. Thin lens

3. Quadrupole

4. PMQ

5. Solenoid

6. Doublet

7. Triplet

8. Bend

9. Edge
Parameter

Q

$f_{x}$

$f_{y}$

$f_{z}$

$B^{\prime}$

Q

$B^{\prime}$

Q

$r_{i}$

$r_{0}$

B

\&

$B^{\prime}$

$\ell$

d

$B_{0}{ }^{\prime}$

lo

d

$\mathrm{B}_{\mathrm{i}}{ }^{\prime}$

$\ell_{i}$

$\alpha$

$\rho$

n

$\beta$

$\rho$

$g$

k

$k_{2}$
Definition

length ( $m m$ )

focal length in $x$-direction $(\mathrm{mm})$

focai length in y-direction ( $\mathrm{mm}$ )

focal length in z-direction ( $\mathrm{mm}$ )

magnetic-field gradient ( $T / \mathrm{m})$

effective length (mm)

maximum magnetic-field gradient ( $T / \mathrm{m})$

physical length of PMQ (mm)

inner radius ( $m m$ )

outer radius ( $\mathrm{mm}$ )

magnetic field ( $G$ )

effective length (mm)

magnetic-field gradient $(T / m)$ in upstream quadrupole

effective length of each quadrupole ( $\mathrm{mm}$ )

distance between quadrupoles ( $\mathrm{mm}$ )

magnetic-field gradient $(T / m)$ in both outer quadrupoles

effective length of outer quadrupole ( $\mathrm{mm}$ )

distance between inner and each outer quadrupule ( $\mathrm{mm}$ )

magnetic-field gradient ( $T / \mathrm{m})$ in inner quadrupole

effective length of inner quadrupole (mm)

angle of bend in horizontal plane (deg)

radius of curvature of central trajectory (mm)

field-gradient index

pole-face rotation angle (deg)

radius of curvature of bend $(\mathrm{mm})$

total gap of magnet ( $\mathrm{mm}$ )

fringe-field factor (default $=0.45$ )

fringe-field factor (default $=2.8$ ) 


10. RF gap $\begin{array}{ll}E_{0} T L \\ & \phi_{5} \\ & \text { egf } \\ & \text { dWf } \\ & h\end{array}$

11. RFQ cell $V / r_{0}^{2}$

AV

L

$\phi_{0}$

type

12. RF cavity

$$
\begin{aligned}
& E_{0} \\
& \phi_{0}
\end{aligned}
$$

13. Tank

$$
\begin{aligned}
& E_{0} T \\
& L \\
& \phi_{5} \\
& n_{C}
\end{aligned}
$$

14. Rotation $\theta$

15. Identical n

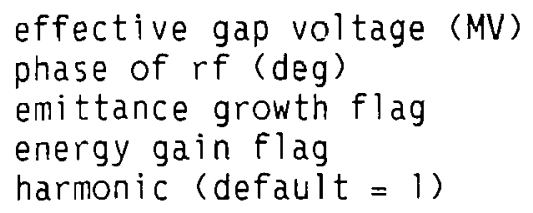

maximum intervane potential difference divided by square of average vane displacement $\left(\mathrm{kV} / \mathrm{mm}^{2}\right)$

product of acceleration efficiency and $\mathrm{ma}_{\mathrm{a}} \mathrm{x} m \mathrm{~m}$ interidis uitaz: (nis cell length $(\mathrm{mm})$ phase of $\mathrm{rf}$ (deg) (see Sec. VI-K)

$=0$; standard cell, no acceleration

$=1$; standard cell, ac:eleration

$=2$; fringe-field, no acceleration

$=3$; fringe-field, acceleration

average accelerating field (MV/m) length ( $\mathrm{mm}$ )

phase of $r f$ (deg) (see Sec. VI-L)

effective acceleration gradient ( $\left.M L^{\prime} / \mathrm{m}\right)$

length ( $\mathrm{mm}$ )

synchronous phase (deg)

number of identical cavities

angle through which coordinate system is rotated about longitudinal axis (deg)

sequence number of element with which this element is identical

V. DYNAMICS CALCULATIONS

The beam matrix is followed through a sequence of transport elements by creating transfer matrices for small segments and calculating

$\sigma=R \sigma_{0} R^{\top}$.

This calculation is done in several different ways, depending on the type of element. 


\section{A. Elements Having Zero Length}

The R-matrix has only three nonzero off-diagonal elements, the impulses to be applied in the three orthogonal planes. Elements of this type are thin lenses, rf gaps, and edge angles on bending magnets.

\section{B. Elements Having Constant Fields and No Energy Gain}

Each element is divided into an integral number of segments. An R-matrix is constructed for a half-segment. For each segment, the beam is transformed to the center of the segment $u$ :ing a R-matrix, a space-charge impulse is applied, and the beam is transformed to the end of the segment using the R-matrix. Elements of this type are field-free drifts, quadrupoles, bending magnets, and solenoids. Doublets and triplets are composite elements of drifis and quadrupoles.

\section{Elements Causing Energy Change cr Having Varying Fields}

Each element is divided into an integral number of segments. For each segment, the beam is transformed by a drift-impulse-drift, maaning that the beam is given a drift transformation to the center of the segment, an impulse that is due to the element and space-charge, and a drift transformation to the end of the segment. Energy charges and phase shifts, if any, are also calculated. Elements of this type are permanent-magnet quadrupoles, RFQ cells, rf cavities, and tanks. In some elements, it is possible to calculate an emittance growth. For example, an rf gap will cause transverse emittance growth because of the phase spread in the beam. Energy spread can cause emittance growth in quadrupoles (chromatic aberrations).

\section{TRANSFER MATRICES}

In this report, the $6 \times 6$ transfer matrices will be partitioned into nine $2 \times 2$ matrices:

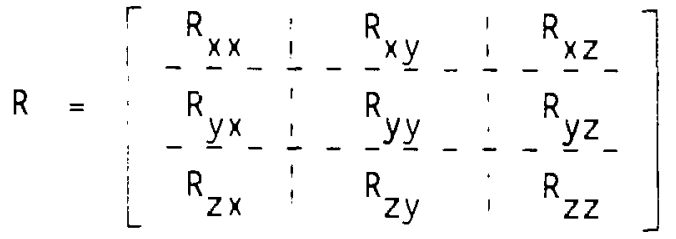


Most of the elements are usually zero, and only the nonzero elements will be defined.

The transfer matrices generated for each element, or for each segment of length $\Delta s$, are defined below. In this section, $B$ and $y$ are the usual relativistic parameters. For elements that involve a cinange in energy, the subscripts $i$ and $f$ will denote values before and after the energy change, and a bar over a quantity will denote an average value.

\section{A. $\quad$ Orift $(l)$}

For a drift length $\Delta s$ in $\mathrm{mm}$, the nonzero elements of the R-matrix are

$R_{x x}=R_{y y}=\left[\begin{array}{cc}1 & \Delta s \\ 0 & 1\end{array}\right]$.

and

$R_{Z Z}=\left[\begin{array}{ll}1 & \Delta S / Y^{2} \\ 0 & 1\end{array}\right]$.

The $\gamma^{2}$ in the dencminator of the 1,2 element of $R_{z z}$ is present because $\Delta v / v=(\Delta p / p) / \gamma^{2}$.

B. Thin Lens $\left(f_{x}, f_{y}, f_{z}\right)$

The focal lengths specified by the thin-lens element are in $\mathrm{mm}$; therefore, the submatrices are

$R_{x x}=\left[\begin{array}{cc}1 & 0 \\ \frac{-1000}{f_{x}} & 1\end{array}\right]$,

$R_{y y}=\left[\begin{array}{cc}1 & 0 \\ \frac{-1000}{f_{y}} & 1\end{array}\right]$,

and

$R_{z z}=\left[\begin{array}{cc}1 & c \\ \frac{-1000 y^{2}}{f z} & 1\end{array}\right]$. 
For any focal length that is zero, the corresponding submatrix is the identity matrix.

C. Quadrupole ( $\left.B^{\prime}, \ell\right)$

For a quadrupole gradient $B^{\prime}$, the $2 \times 2$ transfer matrices in the focusing and tefocusing planes are

$F=\left[\begin{array}{ll}\cos (k \Delta s) & \frac{1}{k} \sin (k \Delta s) \\ -k \sin (k \Delta s) & \cos (k \Delta s)\end{array}\right]$.

and

$D=\left[\begin{array}{ll}\cosh (k \Delta s) & \frac{1}{k} \sinh (k \Delta s) \\ k \sinh (k \Delta s) & \cosh (k \Delta s)\end{array}\right]$,

where

$k=\left[\left|\frac{B^{\prime}}{B p}\right|\right]^{1 / 2}$.

The quantity $B p$ is called the magnetic rigidity of the particle, defined by

$B \rho=\frac{\pi_{0} C B Y}{q}$,

where $q$ and $m_{0}$ are the charge and rest mass of the particle, and $c$ is the velocity of light. The dimensions of $\Delta s$ and $k$ are $m$ and $m^{-1}$, respectively. When $B^{\prime} / B \rho$ is positive, $R_{x x}=F$ and $R_{y y}=D$; when $B^{\prime} / B \rho$ is negative, $R_{x x}=D$ and $R_{y y}=F$. In either case, $R_{z z}$ is the same as for $a$ drift:

$R_{z z}=\left[\begin{array}{ll}1 & \Delta s / \gamma^{2} \\ 0 & 1\end{array}\right]$.

D. $P M Q\left(B^{\prime}, \ell, r_{i}, r_{0}\right)$

The field of a rare-earth cobalt (REC) PMQ of standard design is known analytically and has been verified experimentally. "A discussion of the 
field formula is given in App. E. Typically. PMQs are quite short and have strong pole-tip fields, resulting in "soft-edge" fringe fields that extend beyond the physical eage of the quadrupole for a distance two to three times the inner quadrupole radius. When calculating the field gradient within or near a PMQ, the gradients of all PMQs in the vicinity must be used for determining the total superimposed gradient. The transfer matrices for the impulses that are due to a field gradient $B^{\prime}$ acting over the distance $\Delta$ s are

$R_{x x}=\left[\begin{array}{cc}1 & 0 \\ -k^{2} \Delta s & 1\end{array}\right]$.

and

$R_{y y}=\left[\begin{array}{cc}1 & 0 \\ k^{2} \Delta s & 1\end{array}\right]$,

where

$k^{2}=\frac{B}{B \rho}$

is in inverse meters squared and $\Delta s$ is in meters.

E. Solenoid $(B, 2)$

A derivation of the solenoid transformation is discussed in Ref. 7 . In TRACE $3-D$, the solenoid is divided into small segments of length $\Delta s$, and a submatrix is constructed for the entry, central, and exit sections. This construction is legitimate because all the internal (and nonexistent) entry and exit sections cancel each other. The solenoid is divided into smal? segments so that space-charge forces can be calculated and applied.

The transfer submatrices are

$$
\begin{aligned}
& R_{x x}=R_{y y}=\left[\begin{array}{ll}
C^{2} & \frac{1}{k} S C \\
-k S C & C^{2}
\end{array}\right], \\
& R_{x y}=-R_{y x}=\left[\begin{array}{ll}
S C & \frac{1}{k} s^{2} \\
-k s^{2} & s C
\end{array}\right],
\end{aligned}
$$


and

$R_{Z Z}=\left[\begin{array}{cc}1 & \Delta s / \gamma^{2} \\ 0 & 1\end{array}\right]$.

where

$k=\frac{B}{2 B \rho}$,

$C=\cos (k \Delta s)$,

and

$S=\sin (k \Delta s)$.

The dimensions of $\Delta s$ and $k$ are $m$ and $m^{-1}$, respectively.

F. Doublet $\left(B^{\prime}, \ell, d\right)$

The beam is successively transported with matrices already described for a quadrupole in Sec. VI-C defined by $B^{\prime}, \ell$, a drift length $d$, and another quadrupole defined by $-B^{\prime}, \ell$.

G. Triplet $\left(B_{O}{ }^{\prime}, \ell_{O}, d, B_{j}{ }^{\prime}, \ell_{j}\right)$

The beam is transported with matrices already described in Section VI-C. The outer quadrupoles are defined by $B_{O}{ }^{\prime}$ and $\ell_{0}$, and the inner quadrupole is defined by $B_{i}^{\prime}$ and $\ell_{j}$. The two drifts between outer and inner quadrupoles have length $d$.

H. Bending Magnet $(\alpha, e, n)$

By definition, a positive bend (denoted by $a>0$; bents the particles to the right in the horizontal plane, regardless of the sign of the charge on the particle; a negative a bends particles to the left. The bending radius of the equilibrium orbit is $p$, and $n$ is the field index, given by

$n=-\left[\begin{array}{ll}\frac{e}{B} & \frac{\partial B_{y}}{\partial x}\end{array}\right] \begin{aligned} & x=0 \\ & y=0\end{aligned}$ 
where $B_{y}$ is the vertical component of the magnetic field strength. (The sign of $\rho$ should agree with the sign of $\alpha$. If not, the sign of $\alpha$ will take precedence.) The bending radius $\rho$ is related to $B_{y}$ by

$\rho=\frac{m_{0} c B y}{q B_{y}}$.

The transfer matrix for a horizontal sector magnet is

$$
\begin{aligned}
& R_{x x}=\left[\begin{array}{cc}
C_{x} & \frac{1}{k_{x}} S_{x} \\
-k_{x} S_{x} & C_{x}
\end{array}\right], \\
& R_{y y}=\left[\begin{array}{cc}
C_{y} & \frac{1}{k_{y}} S_{y} \\
-k_{y} S_{y} & C_{y}
\end{array}\right], \\
& R_{z z}=\left[\begin{array}{cc}
1 & -h^{2}\left(k_{x} \Delta s^{2}-S_{x}\right) / k_{x}^{3} \\
0 & 1
\end{array}\right], \\
& R_{x z}=\left[\begin{array}{cc}
0 & h\left(1-C_{x}\right) / k_{x}^{2} \\
0 & h S_{x} / k_{x}
\end{array}\right], \\
& \text { and } \\
& R_{z x}=\left[\begin{array}{cc}
-h S_{x} / k_{x} & -h\left(1-C_{x}\right) / k_{x}^{2} \\
0 & 0
\end{array}\right],
\end{aligned}
$$

where

$$
\begin{aligned}
& h=\frac{1}{|\rho| \frac{\alpha}{|\alpha|},} \\
& k_{x}=\sqrt{(1-n) h^{2}}, \\
& k_{y}=\sqrt{n h^{2}},
\end{aligned}
$$

14 
$C_{x}=\cos \left(k_{x} \Delta s\right)$,

$S_{x}=\sin \left(k_{x} \Delta s\right)$.

$C_{y}=\cos \left(k_{y} \Delta s\right)$,

$S_{y}=\sin \left(k_{y} \Delta s\right) ;$

also,

$\Delta S=|\rho| \Delta \alpha$

is the length of the segment in meters along the equilibrium orbit, and $k_{x}$ and $k_{y}$ are in $m^{-1}$.

I. Edge Angle on Bending Magnet ( $B, p, g, K_{1}, K_{2}$ )

An edge angle is treated as a thin lens, wich the transfer matrix

$R_{x x}=\left[\begin{array}{ll}1 & 0 \\ \frac{1000}{\rho} \tan \beta & 1\end{array}\right]$.

and

$R_{y y}=\left[\begin{array}{cc}1 & 0 \\ \frac{-1000}{\rho} \tan (B-\Psi) & 1\end{array}\right]$,

where $\rho$ is the radius of curvature in $\mathrm{mm}$, and the fringe-field correction angle $\Psi$ is defined by ${ }^{8}$

$\Psi=k_{1} \frac{g}{\rho}\left[\frac{1+\sin ^{2} \beta}{\cos B}\right]\left[1-k_{1} k_{2}\left(\frac{g}{\rho}\right) \tan B\right]$.

Whether or not an edge angle is focusing or defocusing depends not only on the angle $\beta$, but also on the sign of $p$, so caution is advised. The correction angle $\Psi$ is also dependent on the signs of $B$ and $p$. If $K_{1}$ is zero, its default value of 0.45 is used; if $K_{2}$ is zero, its default value of 2.8 is used. 
3. Radio-Frequency Gap ( $E_{O} T L, \phi_{S}$, egf, dWf, h)

In an rf gap, as in RFQ cells, coupled-cavity cells, and coupled-cavity tanks, the energy of the beam would normally change. (There are situations when one does not want acceleration to take place and wants only to consider the focusing and defocusing properties in the gap: namely, when computing the matched input in a periodic system.) When the beam energy is changed, the transversa emittances will change. In this situation, it is convenient to construct the transfer matrix in three separate stages:

1. A transformation that changes the initial $x^{\prime}, y^{\prime}$, and $\Delta p / p$ to $(B y)_{x}$, $(B y)_{y}$, and $\Delta(B y)_{z}$, by multiplying by the initial value of $(B y)_{Z}$, the longitudinal component of the normalized momentum.

2. The impulse transformation that calculates the changes in $\left(B_{y}\right)_{x}$, $(B y)_{y}$, and $\Delta(B y)_{z}$.

3. A transformation back to the $x^{\prime}, y^{\prime}$, and $\Delta p / p$ by dividing by $\left(B_{Y}\right)_{f}$, the final value of the longitudinal momentum.

For an initial kinetic energy, $w_{j}$, the normalized momentum is

$(B \gamma)_{i}=\sqrt{\gamma_{i}^{2}-1}$.

where

$Y_{i}=i+W_{i} / E_{r}$,

with $E_{r}$ being the rest energy of the particle. If dWf $\neq 0$, the energy change in the gap is given by

$\Delta W=|q| E_{0} T L \cos \phi_{3}$

Therefore, the final energy is

$\omega_{f}=\omega_{i}+\Delta \boldsymbol{H} ;$

also,

$\gamma_{f}=1+w_{f} / E_{r}$

and

$\left(B_{Y}\right)_{f}=\sqrt{Y_{f}^{2}-1}$. 
The changes in the normalized momentum components caused by the gap impulse are

$$
\begin{aligned}
& k_{x} x=\frac{-\pi h|u| E_{0} T L \sin \phi_{S}}{m_{0} c^{2} \bar{B}^{2} \bar{\gamma}^{-2} \lambda} x, \\
& k_{y} y=\frac{-\pi h|q| E_{0} T L \sin \phi_{S}}{m_{0} c^{2} \bar{B}^{2} \bar{\gamma}^{2} \lambda} y .
\end{aligned}
$$

and

$k_{z} z=\frac{2 \pi h|q| E_{0} T \sin \phi_{s}}{m_{0} c^{2} \bar{B}^{2} \lambda} z$.

where the bars denote quantities calrulated at the average energy

$\bar{W}=W_{i}+\Delta W / 2$,

and $h$ is the field harmonic (usually $h=1$ ). The field harmonic is used if the $r f$ gap is operated at $h$ times the basic frequency specified by the user (see Sec. IX-A). The total transfer matrix for all three stages is

$$
\begin{aligned}
R_{x x} & =\left[\begin{array}{cc}
1 & 0 \\
0 & (B \gamma)_{f}^{-1}
\end{array}\right]\left[\begin{array}{ll}
1 & 0 \\
k_{x} & 1
\end{array}\right]\left[\begin{array}{ll}
1 & 0 \\
0 & (B \gamma)_{i}
\end{array}\right] \\
& =\left[\begin{array}{ll}
1 & 0 \\
k_{x} /(B \gamma)_{f} & (B \gamma)_{i} /(B \gamma)_{f}
\end{array}\right], \\
R_{y y} & =\left[\begin{array}{ll}
1 & 0 \\
k_{y} /(B \gamma)_{f} & (B \gamma)_{i} /(B \gamma)_{f}
\end{array}\right],
\end{aligned}
$$

and

$$
R_{z Z}=\left[\begin{array}{ll}
1 & 0 \\
k_{z} /\left(B_{\gamma}\right)_{f} & \left(B_{\gamma}\right)_{i} /\left(B_{\gamma}\right)_{f}
\end{array}\right] \text {. }
$$


After computing this R-matrix and using it to calculate the new o-matrix, if the emittance growth parameter egf is nonzero, the elements of the new -matrix are adjusted, as described in App. F, to account for emittance growth in the gap.

K. RFQ (Vir $r_{0}^{2}, A V, L, \phi_{0}$, type)

In an RFQ, as in a cimpled-cavity cell, the rf phase changes continually as the beam moves inough the element. This statement is also true of the beam energyy (unless type $=0$ or 2 ). When the beam arrives at the end of the iement, the rf phase will depend on the length of the element, and on the dynamics as the beam passes through the element. When one has a sequence of RFQ cells, or coupled-cavity cells, the phase shift between adjacent cells is $180^{\circ}$. Therefore, it is more practical to have the parameter $\phi_{0}$ indicate a phase shift rather than an absolute phase. In a sequence of RFQ cells, all of the $\phi_{0}$ parameters will be $-180^{\circ}$ except for the first cell. Before starting the dynamics calculation. TRACE 3-D sets the phase parameter $\phi$ to zero. When the beam arrives at the first RFQ cell, $\phi$ gets changed to $\phi+\phi_{0}$. For the first RFQ cell, $\phi_{0}$ should be the synchronous phase for that cell (for example, $-30^{\circ}$ ).

The beam matrix is followed through the element by a sequence of driftimpulse-drift transformations. The element is divided into 18 equal-length segments (if type $=0$ or 1 ) or into $36 \mathrm{~L} / B \lambda$ segments (if type $=2$ or 3 ). Each of these segments is divided into half lengths of $\Delta s / 2$. In each $\Delta s / 2 \mathrm{drift}$, the phase is incremented by

$\phi=\phi+\frac{2 \pi}{B \lambda} \frac{\Delta s}{2}$,

where $B=V / C$ and $\lambda$ is the free-space wavelength of the rf. If an energy change is permitted (when $t=1$ or 3 ), the longitudinal electric field is calculated, and the new energy is

$w_{f}=w_{i}+|q| E_{z} \Delta s$. 
The average energy is

$\bar{W}=\left(w_{i}+w_{f}\right) / 2$.

The transverse and longiturinal focusing or defocusing forces of the RFQ, and the space-charge defocusing forces, are calculated and applied at the center of the segment. The beam is then drifted for another $\Delta s / 2$, and this process is continued through the element.

In an RFQ cell (specified by type $=0$ or 1 ), the linearized electric field components are

$$
\begin{aligned}
& E_{x}=\left[-\frac{V}{r_{0}^{2}}-\frac{k^{2} A V}{4} \cos k \xi\right] x \sin \phi, \\
& E_{y}=\left[\frac{V}{r_{0}^{2}}-\frac{k^{2} A V}{4} \cos k \xi\right] y \sin \phi,
\end{aligned}
$$

and

$E_{z}=\frac{k A V}{2} \sin k \xi \sin \phi$,

where $k=\pi / L$ and $\xi$ is the local longitudinal coordinate within the cell, defined to be zero at the beginning (upstream end) of the cell. For each drift of length $\Delta s / 2, \xi$ is increased by $\Delta s / 2$. As written, the expressions for $E_{x}$ and $E_{y}$ assume that the horizontal vanes $(x-z$ plane) are closer to the axis at the beginning of the cell. Positive values of the $V / r_{0}^{2}$ parameter indicate this situation; negative values indicate that the vertical vanes are closer to the axis at the beginning of the cell. The sign of $v / r_{0}^{2}$ must alternate in successive RFQ cells.

The changes in $x^{\prime}$ and $y^{\prime}$ that are caused by the RFQ fields, acting over the distance $\Delta s$, are

$$
\Delta\left(x^{\prime}\right)=\frac{\Delta s|g| \sin \phi}{m_{0} c^{2} \bar{B}^{2} \bar{y}}\left[-\frac{v}{r^{2}}-\frac{k^{2} A V}{4} \cos k \xi\right] x,
$$


and

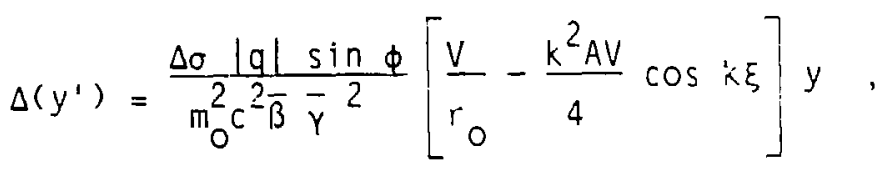

where $\bar{\beta}$ and $\bar{Y}$ are relativistic parameters corresponding to $\bar{W}$.

The change in $\Delta p / p$ caused by a partic s arriving at a particular $\xi$ when the $r f$ phase is $\phi+\Delta \phi$ rather than $\phi$ is

$\Delta(\Delta p / p)=\frac{\Delta s|q|}{m_{0} c^{2} \bar{\beta}^{2} \bar{\gamma}} \frac{\partial E_{z}}{\partial \phi} \Delta \phi$.

Equating the phase displacemer.t $\Delta \phi$ to the longitudinal displacement $z$,

$\Delta \phi=\frac{-2 \pi}{\bar{B} \lambda} z ;$

the change in $\Delta p / p$ is

$\Delta(\Delta p / p)=-\frac{\Delta s \pi|q| k A V \sin k \xi \cos \phi}{m_{0} c^{2} \bar{\beta}^{3} \bar{\gamma} \lambda} z$.

When the type is 2 or 3 , the element is the exit fringe-field region of an RFQ linac. When the specified length $L$ is $2 \beta \lambda$ or longer, this element would be called an exit-radial-matching section because the transverse phase-space ellipses would be very similar at the end of the element. Lengths shorter than $B \lambda / 2$ would be used for controlling the energy change in the fringe-field region.

The electric fields are obtained from the potential function:

$U(r, \theta, \xi)=\frac{V}{2}\left[A T_{0}(r, \xi)+\frac{8}{\left(k_{f} r_{0}\right)^{2}} T_{2}(r, \xi) \cos 2 \theta\right] \sin \phi$,

where

$T_{0}(r, \xi)=\frac{3}{4}\left[I_{0}\left(k_{f} r\right) \cos \left(k_{f} \xi\right)+\frac{1}{3} I_{0}\left(3 k_{f} r\right) \cos \left(3 k_{f} \xi\right)\right]$, 
and

$T_{2}(r, \xi)=\frac{3}{4}\left[I_{2}\left(k_{f} r\right) \cos \left(k_{f} \xi\right)+\frac{1}{27} I_{2}\left(3 k_{f} r\right) \cos \left(3 k_{f} \xi\right)\right]$,

with

$k_{f}=\frac{\pi}{2 L}$.

The modified Bessel functicns $I_{0}$ and $I_{2}$ are replaced by their series expansion, dropping 811 terms in $r$ higher than $r^{2}$ :

$I_{0}\left(k_{f} r\right)=1+\left(k_{f} r\right)^{2} / 4$

$=1+\frac{k_{f}^{2}}{4}\left(x^{2}+y^{2}\right)$,

$I_{2}\left(k_{f} r\right)=\left(k_{f} r\right)^{2} / 8$

Then, using the identity

$r^{2} \cos 2 \theta \equiv x^{2}-y^{2}$.

the potential function can be rewritten in cartesian coordinates, and the electric field components calculated by

$\vec{E}=-\nabla U$.

The results are

$E_{x}=\left[-\frac{v}{r_{0}^{2}} C_{1}(\xi)-\frac{k_{f}{ }^{2} A V}{4} C_{2}(\xi)\right] \times \sin \phi$,

$E_{y}=\left[\frac{V}{r_{0}^{2}} C_{1}(\xi)-\frac{k_{f}{ }^{2} A V}{4} C_{2}(\xi)\right] y \sin \phi$,

and

$E_{z}=\frac{k_{f} A V}{2} s(\xi) \sin \phi$, 
where

$c_{1}(\xi)=\frac{3}{4}\left(\cos k_{f} \xi+\frac{1}{3} \cos 3 k_{f} \xi\right)$.

$c_{2}(\xi)=\frac{3}{4}\left(\cos k_{f} \xi+3 \cos 3_{f} k \xi\right)$.

and

$S(\xi)=\frac{3}{4}\left(\sin k_{f} \xi+\sin 3 k_{f} \xi\right)$

The impulse coefficients for the RFQ fields are

$k_{x}=\frac{\Delta s|q| \sin \phi}{m_{0} c^{2} \bar{\beta}^{2} \bar{y}}\left[-\frac{v}{r_{0}^{2}} c_{1}(\xi)-\frac{k_{f}^{2} A V}{4} c_{2}(\xi)\right]$

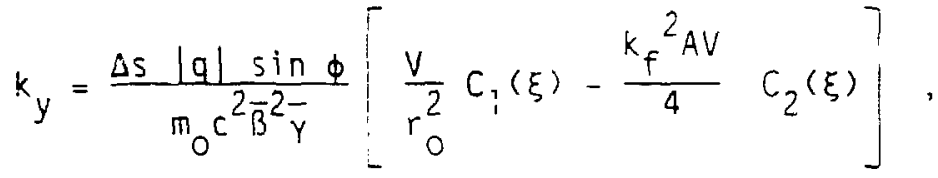

and

$k_{z}=\frac{\Delta s \pi|q| k_{f} A V S(\xi) \cos \phi}{m_{0} c^{2} \bar{B}^{3} \bar{\gamma} \lambda}$.

L. Radio-Frequency Cavity ( $E_{0}, L, \$_{0}$ )

The main purpose of this element is for calculating the motion of a relatively low-velocity electron beam through a coupled-cavity cell. An example of such an element is the side-coupled cavity, often used for electron linacs. When a low-velocity electron enters such a cavity, its velocity can change significantly in one cell. In this case, approximations of constant velocity are not appropriate, and the motion must be obtained by integration through the electromagnetic field in the cavity.

For this purpose, the cell is divided into 18 equal-length segments, $\Delta S=L / 18$. In each segment, the beam is given a drift-impulse-drift transformation. The impulse is calculated at the middle of the segment using the values of the $E_{z}, E_{r}$, and $B_{\theta}$ field components; the beam energy; and the rf phase. The impulse from the space-charge force is also calculated and applied at the middle of each segment. 
The electromagnetic-field components depend on the geometry of the cavity and on $E_{O}$, the average on-axis accelerating field. SUPERFISH ${ }^{9}$ runs have been made for several types of cavities and at various cell lengths for each type. The results are scalable with frequency; therefore, each run at a particular cell length is associated with a value of $\beta=2 L / \lambda$, an implicit assumption for the cell length. For example, SUPERFISH runs were made for the side-coupled cavity geometry used by the racetrack microtron designed by Los Alamos for the National Bureau of Standards. Electric-field information was calculated for cavity lengths having values of $\beta$ from 0.55 to 1.0 in steps of 0.05 . For each value of $B$, the fields near the axis were Fourier analyzed and the first 14 nonzero coefficients were calculated. A table of these coefficients versus $B$ is stored in a TRACE 3-D subroutine, GENFC. Then, for any given cavity length and frequency, a value is determined for $\beta$ based on $L=\beta \lambda / 2$. The Fourier coefficients for this $\beta$ are obtained by a linear interpolation in the table, and the field components are reconstructed from the Fourier coefficients and scaled by $E_{O}$. For details on calculating the electromagnetic-field components in a cavity, and on calculating the linear impulse coefficients, see App. G.

M. Tank $\left(E_{O} T, L, \phi_{S}, n_{C}\right)$

$\rightarrow$ coupled-cavity tank consists of $n_{c}$ identical cavities $L / n_{c}$ long. The quantity $E_{O}{ }^{\top}$ is the constant effective accelerating field, and $\phi_{S}$ is the synchronous phase. Although most of the acceleration takes place in the central portion of each cell, one can think of the rate of energy change as being constant:

$\frac{d W}{d z}=|q| E_{O}^{\top} \cos \phi_{S}$.

In the tank element, each cavity is divided in two halves. In the first half of a cavity, the electromagnetic fields are usually radially focusing and in the second half they are usually radially defocusing. For a synchronous phase near zero, these two effects essentially cancel each other when ions are accelerated, but electrons can have a net inward motion. At each cavity in a tank, the beam is drifted to the middle of the first half of the cavity, given an impulse based on field quantities averaged over the first half of the cavity and on the space-charge forces, and then drifted to the center of the 
cavity. This procedure is repeated for the second half of the cavity and for all cavitys in the tank. The details for calculating the impulse coefficients are given in App. H.

N. Special ( $\ell$, user-defined parameters)

This element provides a mechanism for the user to insert any special element of his choice. Of the five parameters availabla to define this element, the first must be the total length of the element and the remainirg four are arbitrary. The length is needed by the graphics display when the beam profiles are drawn. If more parameters are needed, up to 10 can be input into EXTRA, an array designated for holding extra parameters.

The user must supply a subroutine called SPECIAL, with arguments $P$ and NEL where $P$ is an array containing the five possible parameters and NEL is the sequence number of this element.

\section{Rotation $(\theta)$}

The transverse coordinates may be rotated through an angle $\theta$ (deg) about the longitudinal axis. Thus a rotated element (such as a bending magnet, quadrupole, doublet, or triplet) may be inserted into a transport system by preceding and following the element with the appropriate coordinate rotation. A positive angle rotates the beam clockwise (looking at the $x-y$ plane from the positive $z$-direction), simulating a counterclockwise rotation of the elements that follow it.

The rotation is accomplished by the transfer matrix

$R=\left[\begin{array}{rrrrrr}C & 0 & S & 0 & 0 & 0 \\ 0 & C & 0 & S & 0 & 0 \\ -S & 0 & C & 0 & 0 & 0 \\ 0 & -S & 0 & c & 0 & 0 \\ 0 & 0 & 0 & 0 & 1 & 0 \\ 0 & 0 & 0 & 0 & 0 & 1\end{array}\right]$,

where $C$ and $S$ denote $\cos \theta$ and $\sin \theta$, respectively. 
This element gives the user an easy way to duplicate any element defined in the transport system. The only parameter required is the sequence number of the element with which it is identical. For example, if there are several quadrupoles in the system that all have the same characteristics, only one needs to be specif: ally defined by a type code anc parameter set; the rest can be included using the identical element. When any parameter in the defined quadrupole is modified, all quadrupoles defined by the identical element are likewise modified. The sequence number defining the identical element must not be the sequence number of another identical element.

\section{SPACE-CHARGE IMPULSES}

Approximate expressions for the electric field components that are due to a uniformly charged ellipsoid, as given by Lapostolle, ${ }^{10}$ are

$$
\begin{aligned}
& E_{x}=\frac{1}{4 \pi \varepsilon_{0}} \frac{3 I \lambda}{c y^{2}} \frac{(1-f)}{r_{x}\left(r_{x}+r_{y}\right) r_{z}} x, \\
& E_{y}=\frac{1}{4 \pi \varepsilon_{0}} \frac{3 I \lambda}{c y^{2}} \frac{(1-f)}{r_{y}\left(r_{x}+r_{y}\right) r_{z}} y,
\end{aligned}
$$

and

$E_{z}=\frac{1}{4 \pi \varepsilon_{0}} \frac{3 I \lambda}{c y^{2}} \frac{f}{r_{x} r_{y} r_{z}} z$,

where $r_{x}, r_{y}$, and $r_{z}$ are the semiaxes of the ellipsoid; I is the average particle current (the average electrical current is $I|q|$ ), assuming that a bunch occurs in every period of the if; $\lambda$ is the free-space wavelength of the $r f$; $c$ is the velocity of light; and $\varepsilon_{0}$ is the permittivity of free space. The form factor $f$ is a function of $p \equiv \gamma r_{z} / \sqrt{r_{x}{ }^{r}}$. Values for $f$ are given in Table II for specific values of $p$ and $1 / p$. 
The change in the normalized momentum components caused by applying the space-charge force during the time interval required for the beam to move a distance $\Delta s$ is

$\Delta(B \gamma)_{u}=\frac{q E_{U} \Delta s}{m_{0} c^{2} B}$.

where $u$ represents $x, y$, or $z$.

TABLE II

SPACE-CHARGE FORM FACTOR

$p \equiv Y r_{z} / \sqrt{r_{x} r_{y}}$

$\begin{array}{llll}\frac{p}{p} & \frac{f}{1 / p} & f \\ 0.00 & 1.000 & 0.00 & 0 \\ 0.05 & 0.926 & 0.05 & 0.007 \\ 0.10 & 0.861 & 0.10 & 0.020 \\ 0.15 & 0.803 & 0.15 & 0.037 \\ 0.20 & 0.750 & 0.20 & 0.056 \\ 0.25 & 0.704 & 0.25 & 0.075 \\ 0.30 & 0.661 & 0.30 & 0.095 \\ 0.35 & 0.623 & 0.35 & 0.115 \\ 0.40 & 0.588 & 0.40 & 0.135 \\ 0.45 & 0.556 & 0.45 & 0.155 \\ 0.50 & 0.527 & 0.50 & 0.174 \\ 0.55 & 0.500 & 0.55 & 0.192 \\ 0.60 & 0.476 & 0.60 & 0.210 \\ 0.65 & 0.453 & 0.65 & 0.227 \\ 0.70 & 0.43 i & 0.70 & 0.244 \\ 0.75 & 0.4 ' 3 & 0.75 & 0.260 \\ 0.80 & 0.294 & 0.80 & 0.276 \\ 0.85 & 0.378 & 0.85 & 0.291 \\ 0.90 & 0.362 & 0.90 & 0.306 \\ 0.95 & 0.347 & 0.95 & 0.320 \\ 1.00 & 0.333 & 1.00 & 0.333\end{array}$


The above formulation is valid only for ellipsoids that are upright with respect to the local coordinate system. When the beam passes through a solenoid or a bending magnet, it is possible for the ellipsoid to become tilted with respect to the local coordinate system. In this case, the beam ellipsoid must first be transformed to a coordinate system in which it is upright before calculating and applying the space-charge impulses. The ellipsoid is then transformed back to the local coordinate system.

A tilted ellipsoid is indicated if the $\sigma$-matrix elements $\sigma_{13}, \sigma_{35}$, or $\sigma_{51}$ are nonzero. If $\sigma_{13}$ is nonzero, the ellipsoid is tilted in the $x-y$ plane. The angle between the $x$-axis and the axis of the elliptical projection on the $x-y$ plane is

$\theta=\frac{1}{2} \tan ^{-1} \frac{2 \sigma_{13}}{\sigma_{33}-\sigma_{11}}$

The ellipsoid can be brought upright with respect to the $x-y$ plane by a rotation of $-\theta$, accomplished by applying the transfer matrix

$R=\left[\begin{array}{rrrrrr}C & 0 & -S & 0 & 0 & 0 \\ 0 & C & 0 & -S & 0 & 0 \\ S & 0 & C & 0 & 0 & 0 \\ 0 & S & 0 & C & 0 & 0 \\ 0 & 0 & 0 & 0 & 1 & 0 \\ 0 & 0 & 0 & 0 & 0 & 1\end{array}\right]$,

where $C$ and $S$ denote $\cos \theta$ and $\sin \theta$. The rotated ellipsoid can be checked to see if it is upright with respect to 'he $y-z$ plane. If not, it can be rotated by a similar transfer matrix. It can be rotated a third time, if necessary, to make it upright with respect to the $z-x$ plane.

When the beam is in the upright position, the space-charge impulses can be calculated and applied, and the three rotations given in the reverse directions in the reverse order.

Before the initial rotations are made, the ellipsoid is expanded in the z-direction by applying a transfer matrix that is equal to the identity matrix except that the 5,5 element is the relativistic parameter $Y$. After the final rotation described above, the ellipsoid is contracted by the inverse of the perturbed identity matrix discussed above. 


\section{USER INSTRUCTIONS}

In addition to the input and output filts associated with the graphics terminal, TRACE 3-D uses tape10, tape20, tape30, and tape31. Tape10 is a binary (unformatted) file written by TRACE 3-D whenever a parameter is changed (through the "input" command) and after every dynamics run. This file contains all the information needed for restarting the program if, for whatever reason, it has been terminated. Tape30 is an ASCII input file containing the initial value of various parameters, including beam characteristics and transport-system parameters. Tape20 and tape31 are described below.

Upon running TRACE 3-D, the message "enter 3 to read tape30 or 1 to read tapel0" will appear on the graphics terminal. Unless a tapelo exists and the user is restarting the program, the normal response would be to enter a "3", followed by a "return." The information on tape 30 is read by a single READ (30, DATA) statement, using the namelist feature of the FORTRAN Extended Compiler for Control Data operating systems. This feature is extremely useful because it allows one to enter a few or all of the parameters defined in the NAMELIST statement.

After reading the initial input values, a few constants are initialized, the terminal is put in the graphics mode, and a cursor (cross hairs) will appear on the screen. The program is waiting for a command from the user. $A$ command is issued by striking 1 of 18 alphabetic characters on the keyboard. (Some terminals require a "carriage return" to be struck after entering a letter, and some do not.) The program performs the task specified by the character. After the task has been completed, the cursor is again displayed and the program awaits another command. Table III gives the commands recognized by TRACE 3-D.

The user terminates the program by typing an "e" for "end." Information is then written on tape 31 in a format suitable to be used as input data (tape30) for a future run.

The other file mentioned earier, tape20, is a smali file used to facilitate the namelist feature after the "i" (input) command. After this command, a prompt "?" appears, asking the user to type input information on the screen followed by a return. This information is read using an A-format and is written on tape20 after appending the characters "\$DATA" at the 
beginning and a "\$" at the end of the character string. Tape20 is then rewound and read with a READ (20, DATA) statement.

TABLE III

COMMANDS RECOGNIZED BY TRACE 3-D

Command

\begin{tabular}{|c|c|}
\hline a & $(a d d)$ \\
\hline b & (beam) \\
\hline d & (delete) \\
\hline e & (end) \\
\hline$f$ & (phase) \\
\hline g & (go) \\
\hline i & (input) \\
\hline j & (projections) \\
\hline$\ell$ & (ellipse) \\
\hline m & $(\operatorname{match})$ \\
\hline o & (mismatch) \\
\hline$p$ & (print) \\
\hline$r$ & $(R-\operatorname{matr} i x)$ \\
\hline S & (save) \\
\hline$t$ & (trace) \\
\hline $\mathrm{u}$ & (update) \\
\hline w & $(\phi-W)$ \\
\hline$z$ & $(\sigma-\operatorname{matr} i x)$ \\
\hline
\end{tabular}

Action

add (insert) elements in transport system

print beam parameters

delete elements from transport system

terminate the program

calculate and print phase advances

draw graphics background and follow beam through transport system

enter new parameters

plot initial and final beam projections on the

$x-y, \quad x-z$, and $x-\Delta p / p$ planes

determine emittance ellipses from three profile measurements

perform matching specified by the mt parameter

calculate and print mismatch factors

print parameters for beam, control, graphics, and transport

print R-matrix from latest run

save ellipse parameters and o-matrix

follow the beam through a sequence of elements and display results on existing graph

replace ellipse parameters and o-matrix by their stored values

print phase and eneigy information

print modified a-matrix

IX. INPUT VARIABLES

Before giving a detailed description of the action taken when each command is issued, all of the input variables will be defined. The internal names of the variables will be written in capital letters. If the variable is a dimensioned array, the dimensions as appearing in a DIMENSION or COMMON 
statement will be enclosed in parenthesis. The variables can be grouped in several categories: transport system, beam characteristics, control

parameters, matching parameters, graphics scales, print parameters, and extra parameters.

A. Transport system [NT(NELMAX), A(5, NELMAX), FREQ, PQEXT, and ICHROM]

The elements of a transport system are defined in sequential order by a "type code" in the NT array and by the associated parameters (five or fewer) in the A array. The quantities have been defined earlier in Sec. IV. (The maximum number of elements. NELMAX, is defined in the FORTRAN program by PARAMETER statements.) FREQ is the frequency of the if in MHz. Even if no if elements appear in the transport system. FREQ is necessary for defining the length of the beam bunch, which is specified in degrees at this frequency. PQEXT defines the extension of the finge field in PMOs. These fields will be calculated for a distance of PQEXT times $r_{i}$ from each edge of a PMQ, where $r_{i}$ is the inner radius of the PMQ. ICHROM is a flag that, if nonzelo, specifies that chromatic aberrations are to be taken into account when the beam passes through a thin lens, a PMQ, and a quadrupole (and, therefore, a doublet and a triplet). Chromatic aberrations cause an effective emittance growth, so the o-matrix is modified as described in App. I.

B. Beam Characteristics [ER, $Q, W, X I, \operatorname{BEAMI}(6), \operatorname{EMITI}(3), \operatorname{SIGI}(6,6)]$

$E R$ is the rest energy of the particles, and $W$ is their kinetic energy (units in MeV); $Q$ is the charge state ( +1 for protons), and $X I$ is the beam current in MA. The initial ellipse parameters are in the BEAMI array in the order $\alpha_{x}, \beta_{x}, \alpha_{y}, \beta_{y}, \alpha_{\phi}, \beta_{\phi}$. These are the Courant-snyder, or Twiss, parameters for the initial phase-space ellipses in the three phase planes. The $\alpha^{\prime} s$ are dimensionless, $\beta_{x}$ and $\beta_{y}$ are in meters (or $\mathrm{mm} / \mathrm{mrad}$ ), and $\beta_{\phi}$ is in deg/key. The initial emittances in the $x-x^{\prime}, y-y^{\prime}$, and $\Delta \phi-\Delta W$ phase spaces are in the EMITI array. The units are in $\pi \cdot m m \cdot m r a d$ in the $x-x^{\prime}$ and $y \cdot y^{\prime}$ planes, and in $\pi \cdot d e g \cdot k e V$ in longitudinal phase space. When spacecha, ge forces are included (when $X I \neq 0$ ), these emittances should be five times the rms emittances. SIGI is a $6 \times 6$ array containing the initial o-matrix. The elements of the SIGI array usually are not, but can be, input quantities. 
C. Control Parameters (N1, N2, SMAX, PQSMAX, IBS, NEL1, NEL2)

$\mathrm{N} 1$ and $\mathrm{N} 2$ are the sequence numbers of the transport elements through which the beam is followed. When the beam is followed in the forward direction (normally the case), the beam starts at the beginning (upstream end) of Element $\mathrm{Nl}$ and is followed to the downstream end of Element N2. If $\mathrm{N} 1$ > N2, the beam starts at the downstream end of Element $N 1$ and is followed to the upstream end of Element $N 2$. A negative value in Element $N 2$ tells the program to follow the beam backward through Element N1. SMAX is the maximum step size (in $\mathrm{mm}$ ) for the dynamics calculations in most of the elements. Near PMQs, the maximum step size is PQSMAX (mm), usually a smaller value than SMAX. In some elements, the step size is set automatically. For example, each RFQ cell and coupled-cavity is divided into 18 segments, and each cavity in a tank is divided into 2 parts. IBS is a flag indicating how the initial o-matrix is generated. If IBS $=0$ (the normal case), the elements of the initial o-matrix are calculated from the BEAMI and EMITI arrays. If IBS $\neq$ 0 , the initial o-matrix is assumed to be in the SIGI array.

NELI and NEL2 are the sequence numbers of the first and last transport elements to be plotted and printed. Their default values are 1 and NELMAX. These parameters allow the user to select small segments of a transport system that would have too many elements to plot legibly on one display.

D. Matching Parameters $[M T, N C, \operatorname{MP}(2,6), \operatorname{MVC}(3,6), \operatorname{BEAMF}(6), \operatorname{DELTA}$, $\operatorname{IJM}(2,6), \operatorname{VAL}(6), \mathrm{NIT}]$

MT specifies the type of matching desired. Types 1 through 4 specify that the matched-ellipse parameters are to be found for a periodic system. Types 5 through 9 indicate that values are to be found for specified element parameters, called variables, that cause the beam ellipse parameters, R-matrix elements, or modified $\sigma$-matrix elements to satisfy specified conditions at the end of Element $\mathrm{N}_{2}$. The meanings of the type codes are given below.

$$
\begin{aligned}
& \text { MT }=1 \text { : determines matched values of } \alpha_{x}, \beta_{x}, \alpha_{y}, \text { and } \beta_{y} . \\
& M T=2: \text { determines matched values of } \alpha_{\phi} \text { and } \beta_{\phi} . \\
& M T=3: \text { determines matched values of } \beta_{x}, \beta_{y}, \text { and } \beta_{\phi} \text {, assuming } \\
& \text { that } \alpha_{x}=\alpha_{y}=\alpha_{\phi}=0 . \\
& \text { MT }=4: \text { determines matched values of } \alpha_{x}, \beta_{x}, \alpha_{y}, \beta_{y}, \alpha_{\phi} \text {, and } \beta_{\phi} . \\
& \\
& \text { values for } \alpha_{x} \text { and } \beta_{x} .
\end{aligned}
$$


MT $=6:$ determines vaiues of the variables $(\leq 2)$ that produce specified values for $\alpha_{y}$ and $\beta_{y}$

MT $=7$ : determines values of the variables ( $\leq 2)$ that produce specified values for $\alpha_{\phi}$ and $\beta_{\phi}$.

MT $=8$ : determines values of the variables $(\leq 4)$ that produce specified values for $\alpha_{x}, \beta_{x}, \alpha_{y}$, and $\beta_{y}$.

$M T=9$ : determines values of the variables $(\leq 6)$ that produce specified values for $\alpha_{x}, \beta_{x}, \alpha_{y}, \beta_{y}, \alpha_{\phi}$, and $\beta_{\phi}$.

$M T=10$ : determines values of the variables ( $\leq N C)$ that produce specified elements of the R-matrix.

$M T=11$ : determines values of the variables ( $\leq N C$ ) that produce specified values of specified elements of the modified $\sigma$-matrix (defined in Sec. X under the " $z$ " command).

NC is the number of conditions to be satisfied by the matching procedure and is automatically set by the program for matching types 1 through 9 . The user must specify a value $(\leq 6)$ for NC for matching types 10 and 11 . The MP array contains the parameter and element numbers of the variables for matching types 5 through 11 . MP $(1, n)$ contains the parameter number $(1$ to 5$)$ and $\operatorname{MP}(2, n)$ contains the element number ( 1 to NELMAX) for the $n$th variable. Ideaily, the number of variables, NV, should equal the number of conditions, NC. When either $M P(1, n)$ or $M P(2, n), n \leq N C$, is not within its legitimate range, that variable is not properly defined and is ignored. Matching is attempted even though NV: NC.

It is possible to "couple" one transport element parameter to each variable parameter. For example, two drifts may be coupled so that when one is increased the other is decreased by the same amount, keeping the total distance constant. Or two drifts may be coupled so that they are both changed by the same amount, a feature that could be used for maintaining symmetry. The other type of coupling allowed is a proportional change in a parameter. For example, two quadrupole gradients may be coupled so that when one changes, the cther changes b; the same proportion. The indices of the coupled parameters and $k$, the type of coupling, are specified in the IVC array. $\operatorname{MVC}(1, n)$ and $\operatorname{MVC}(2, n)$ contain $i$ and $j$, the parameter and element number, respectively, of the transport parameter $A(i, j)$ that is coupled to the $n$th variable. $M v C(3, n)$ contains $k$, ither +1 or -1 . If the coupled variables are drifts, $k=-1$ keeps the sum of the two drifts constant, and $k=+1$ changes 
both drifts by the same amount. If the coupled variables are not drifts, when the value of the nth variable is changed from $x_{1}$ to $x_{2}$, the coupled parameter is multiplied by $k x_{2} / x_{1}$.

The BEAMF array contains the values wanted (for MT = 1 through 9) for some or all of the ellipse parameters $\alpha_{x}, \beta_{x}, \alpha_{y}, \beta_{y}, \alpha_{\phi}$, and $\beta_{\phi}$ at the end of Element $\mathrm{N}_{2}$. For $M T=1$ through 4 , the values in BEAMF are adjusted by the program; for $M T=5$ through 9, the values in BEAMF are set by the user. The indices for the R-matrix elements for $M T=10$ are in the IJM array, and the desired values for these elements are in the $\operatorname{VAL}$ array. IJM $(1, n)$ contains the $i$-index (row), IJM $(2, n)$ contains the $j$-index (column), and VAL ( $n$ ) contains the desired value for Rij for the $n$th condition, where $n \leq N C$. These same arrays are used for $M T=11$, except that they refer to the indices and values of the modified $\sigma$-matrix. A convergence factor is calculated each iteration in the matching procedure. For matching types 1 through 9 , the convergence factor is the largest of the mismatch factors calculated for the ellipse parameters in the appropriate planes. (For a definition of ellipse mismatch factors, see App. C.) For matching types 10 and 11 , the convergence factor is the largest difference between the values desired and the values obtained for specified elements of the R- or $\sigma$-matrices. (If the specified value has a magnitude greater than one, a relative difference is used.) DELTA is a convergence criterion defaulted to 0.0001 . When the largest of the mismatch factors is less than or equal to DELTA, the solution is assumed to be close enough and the procedure $i$ : serminated. The procedure is also terminated if convergence has not been achieved after NIT iterations. NIT is defaulted to 10 , but may be changed by the user.

E. Graphics Scales (XM, XPM, YM, DPM, DWM, DPP)

These quanities set values for the boundaries of the phase-space and profile plots. XM and XPM set limits on the transverse phase-space plots in $\mathrm{mm}$ and mrad, respectively, and $Y M(\mathrm{~mm})$ sets the limit on the transverse-profile plots. DPM (deg) and DWM (keV) are the limits of the longitudinal phase-space plots, and De? (deg) is the limit on the phase-profile plot. 
F. Print Parameters [NPRIN, IJPRIN(2,20)]

Up to 20 transport parameters can be written on the graphics display to identify the results with the more important parameters. NPRIN is the number of parameters to print, and the $i$ and $j$ indicies of parameter $A(i, j)$ are in IJPRIN $(1, n)$ and IJPRIN $(2, n)$ for the $n$th parameter.

\section{G. Extra Parameters [NXTRA, EXTRA(10)]}

Some additional storage space is reserved for any extra parameters, and NXTRA of these parameters in the array EXTRA will be printed by the "p" (see Sec. X) command. One use for this EXTRA array would be to provide a place for the user to store additional parameters needed for defining a special element.

\section{$X$. DESCRIPTION OF COMMANDS}

The following description of the action taken when each command is given, rather than being in alphabetical order, will be in an order in which the user might reasonably issue the commands.

To print most of the data file on the graphics terminal, type "p". The location of the cross hairs determines the scope position at which the printing starts. It is a good idea to give this command in the beginning and, after any complicated input sequence, to check the accuracy of the data. When a dynamics run gives unrealistic results, or when a matching procedure is getting nowhere, something is probably wrong with the data.

If the printed output comes too close to the bottom of the graphics display, printing will stop and the cursor will be displayed. Printing may be resumed at the cursor by entering a "c". Any other command will cause the program to exit from the print mode and to wait for another command.

If the data appear to be correct, perform the dynamics calculations by typing " $g$ ". The scope is erased and the graphics background is drawn. The initial phase-space ellipses are drawn, the horizontal (solid) ellipse and vertical (dashed) ellipse on the same background, and the longitudinal (also dashed) ellipse on a separate background. If the beam is going forward, the initial ellipses (defined by arrays BEAMI and EMITI) will be drawn at the upper left; if the beam goes backward, the ellipses will be drawn at the upper right. As the beam is followed through the specified elements, the envelopes are plo ted on the lower part of the screen: the horizontal (solid line) and phase (dots) on the upper half and the vertical (dished line) on the lower 
half. When the beam reaches its destination, the final ellipses (defined by ellipse parameters in array BEAMJ and emittances in array EMITO) are drawn and the following information is written in the space between the initial and final ellipses: the beam current; the initial and final emittances; the initia! and final energies; the values of the NPRIN parameters, if any.

To see how a particular parameter affects the beam, one or more values can be changed by getting into the input mode by typing "i". The location of the cross hairs specifies the point on the screen at which the input will be typed. After a "?" appears on this spot, type one or more parameter names and their new values using the format

NAME $1=$ value 1, NAME2 $=$ value $2, \ldots,$.

Sequential values in an array can be entered without retyping the array name. For example, to change the third and fourth parameters in the fifth element, type

$f(3,5)=1 ., 2$.

followed by a return. Any of the quantities defined previously in Sec. IX may be entered. Any typing mistake detected by the namelist read will cause the entire line to be rejected, and an error message will appear asking the user to try again or to exit from the input mode. Each input line is limited to 100 characters.

After making some changes, one would usually want to see the effect on the beam. Typing "t" causes the new input ellipses, the new profiles, and the new final ellipses to be drawn on top of the previous ones, providing an easy comparison.

To find the matched ellipse parameter in a periodic system, or to find the values of some transport parameters that match the beam or give the desired values for specified elements of the R- or o-matrices, type " $m$ ". Depending on the matching type, specified by MT, a solution is sought for a set of NC nonlinear, simultaneous equations. The method used is that of regula falsi, an iterative procedure that, starting with an initial "guess" for the solution, usually converges to a solution in a reasonable number of trials. (If the value of any variable is zero, that value will not be 
changed.) At each iteration, the convergence factor and the values of the variables are printed on the screen, starting at the position of the cross hairs. If a solution has not been found after NIT iterations, the variables will be set to their "best" values, those that give the minimum convergence factor. To try again for NIT iterations, starting with the best results as the initial guess, issue the " $m$ " command again. If no progress is made toward a solution after several of these tries, there are several possible reasons:

1. Something is wrong with the data. Check this by issuing the "p" command.

2. The solution is too far from the initial guess to be found by this technique. Using the " $i$ " command, put in different parameters and try again.

3. No colution exists. In this case, the transport system may have to be modified, for example by adding more elements.

4. If the number of variables is less than NC, an exact solution probably does not exist.

To insert one or more elements between any two elements, type "a". At the location of the cross hairs, the message "enter ml, m2 cinsert ml elements before Element m2)" will appear. (If this command has been issued by mistake, enter a negative number for $\mathrm{mi}$ and anything for $\mathrm{m} 2$.$) To insert two new$ elements between Elemenis 5 and 6 , enter 2,6 (a comma or a space can be used to separate the two integers). The program will move all type codes (in array NT) and all transport parameters (in array $A$ ), starting at Element m2, to locations increased by m1. [NT(6) is moved to NT(8), etc.] The program is then put in the input mode, just as if the user had issued an " $i$ " command. Enter any needed parameters, including correct element type in the NT array, followed by a return.

To remove one or more elements, type "d". At the location of the cross hairs, the message "enter $\mathrm{ml}$, m2 (delete $\mathrm{ml}$ elements starting with Element m2)" will appear. (If this command has been issued by mistake, enter a negative number for $\mathrm{ml}$ and anything for $\mathrm{m} 2$.) To remove Elements 6 and 7 , enter 2,6. All type codes (in array NT) and all transport parameters (in array A), starting at Element $m 2$, will be moved up by ml elements.

Sometimes it is useful to save the ellipse parameters by typing " $s$ ". The following message will appear at the location of the cross hairs: "enter two numbers, which beam to save and where. BEAMI =1, BEAMF=2, BEAMO=3. Five positions available." After receiving a prompt "?", the user should enter two 
integers separated by a comma or a space. For example, to save BEAMI in the third of the five storage areas provided, enter 1,3. The six ellipje parameters in BEAMI will be stored in the array HOLJ $(i, 3), i=1,6$. At the same time, the initial o-matrix, contained in the array SIGI, is stored in the third of five storage arrays $\operatorname{SIGS}(i, j, 3), i=1,6 ; j=1,6$. Also, the initial kinetic energy $W$ is stored in WS(3). When the beam is followed from Element $\mathrm{N} 1$ through Element $\mathrm{N} 2$, the final ellipse parameters and the $\sigma$-matrix are in the arrays BEAMO and SIG, and the final kinetic energy is in WW. These quantities can be saved in the second storage positions, $\operatorname{HOLD}(i, 2)$, SIG $(i, j, 2)$, and WS(2), by issuing the "s" command and entering 3,2 .

If one wants to follow the beam from the end of Element $\mathrm{N} 2$ through another element farther downstream (or upstream, for that matter), the initial ellipse parameters, o-matrix, and energy can be loaded from the second storage positions by typing "u". At the location of the cross hairs, the message "enter two numbers, which beam to restore and whence $B E A M I=1$, $B E A M F=2$. Five holding positions possible." To load BEAMI, SIGI, and Wrom the second storage positions, enter 1,2 after receiving the prompt.

To see what is stored in all beam arrays and storage positions, issue the command " $b$ ". At the cross-hairs position, the BEAMI, BEAMF, BEAMO, and HOLD arrays are printed.

Whenever the beam is followed between $N 1$ and $N 2$, by either " $g$ " or " $t$ " commands, the transfer matrix for this distance is stored in the array RM. To see what is in RM, type " $r$ ". The $6 \times 6$ transfer matrix will be printed starting at the cross hairs.

If the beam has just been followed through one period of a periodic structure, the phase advances and the matched ellipse parameters can be calculated from the R-matrix by giving the commarid "f". The phase advance and the $\alpha$ and $\beta$ ellipse parameters for each of the three phase-space planes are calculated, assuming the $2 \times 2$ R-matrix for each plane cain be written in the form

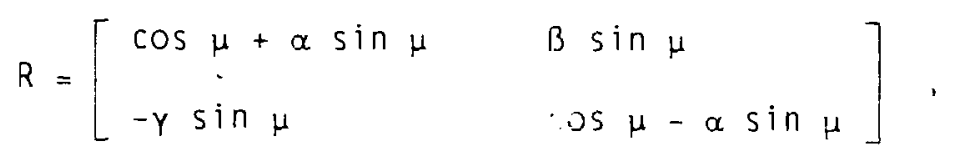

where $y=\left(1+\alpha^{2}\right) / \beta$. The parameters are printed at the position specified by the cross hairs. 
Several other print options are available. One is a print of the mismatch factors, obtained by typing " $O$ ". The mismatch factors between the ellipses defined by the parameters in the BEAMO array and the parameters in the BEAMF array are calculated and printed at the location of the cross hairs. The definition of the mismatch factor and the explanation of how it is calculated are given in App. C.

Some useful information about the longitudinal plane can be printed by typing " $w$ ". At the position of the cross hairs, the following information is printed: the phase and energy of the beam center, the phase and energy half-widths, the length (mm) of the longitudinal semiaxis, the $\Delta p / p$ half-width (mrad), and the longitudinal emittance in the $z-\Delta p / p$ plane $(\pi \cdot m m \cdot m r a d)$.

The command " $z$ " prints the modified s-matrix in the same format used in TFANSPORT, namely:

\begin{tabular}{|c|c|c|c|c|}
\hline$x^{\prime} \max$ & $r 12$ & & & \\
\hline$y_{\max }$ & 113 & $r^{2} 23$ & & \\
\hline$y^{\prime} \max$ & r 14 & 124 & $r 34$ & \\
\hline$z_{\max }$ & 115 & 125 & $r 35$ & $r 45$ \\
\hline$(\Delta p / p)$ max & $r 16$ & $r_{25}$ & 136 & $r_{46}$ \\
\hline
\end{tabular}

The maximum extent of the beam ellipsoid in the ith dimensions is given by $\sqrt{\sigma_{i} i}$. The correlations between the various coordinates are defined by $r_{i j}=\sigma_{i j} / \sqrt{\sigma_{i i} \sigma_{j j}}$

Because $\sigma$ is a symmetric matrix, only half of the $r_{i j}$ 's need be printed. The command " 2 " provides a mechanism for determining the ellipse parameters and emittances in the two transverse phase plaries from measurements of beam sizes at three locations. When this command is given, the message "determine emittances from three width measurements" anpears on the screen. The locations of the measurements are to be (or may have been) specified by the user by three element numbers in the array LOC. The measurements are 
assumed to be at the downstream end of the specified elements. If $\operatorname{LOC}(1)=0$, the first measurement is at the beginning of the first element. If LOC(2) or LOC $(3)$ is not greater than zero, the program assumes that the information on the locations and beam widths has not been input, and the user will be asked to do so by the message "enter loc(3), xhw(3), yhw(3), (1or'1).1t.0 aborts.)". The user should then enter three values in LOC, three horizontal beam half-widths in array XHW, and three vertical beam half-widths in array YHW. Before issuing this command, the graphics display should be on the screen because measured widths will be plotted and beam profiles will be drawn as the progran goes through five iterations in the attempt to solve the problem. The details of the procedure are described in App. J. If the solutions to the equations result in a negative value for the emittance in either plane, the message will be written "unrealistic solution. check data."

The command " $j$ " causes the initial and final $x-y, x-z$, and $x-\Delta p / p$ projections of the beam ellipsoid to be displayed.

\section{EXAMPLES}

A. Matching Between 400-MHz RFQ and DTL

This example is the one mentioned in the introduction. Figure 1 shows an input fil: to be read as Tape 30 as described in Sec. VIII. The input file

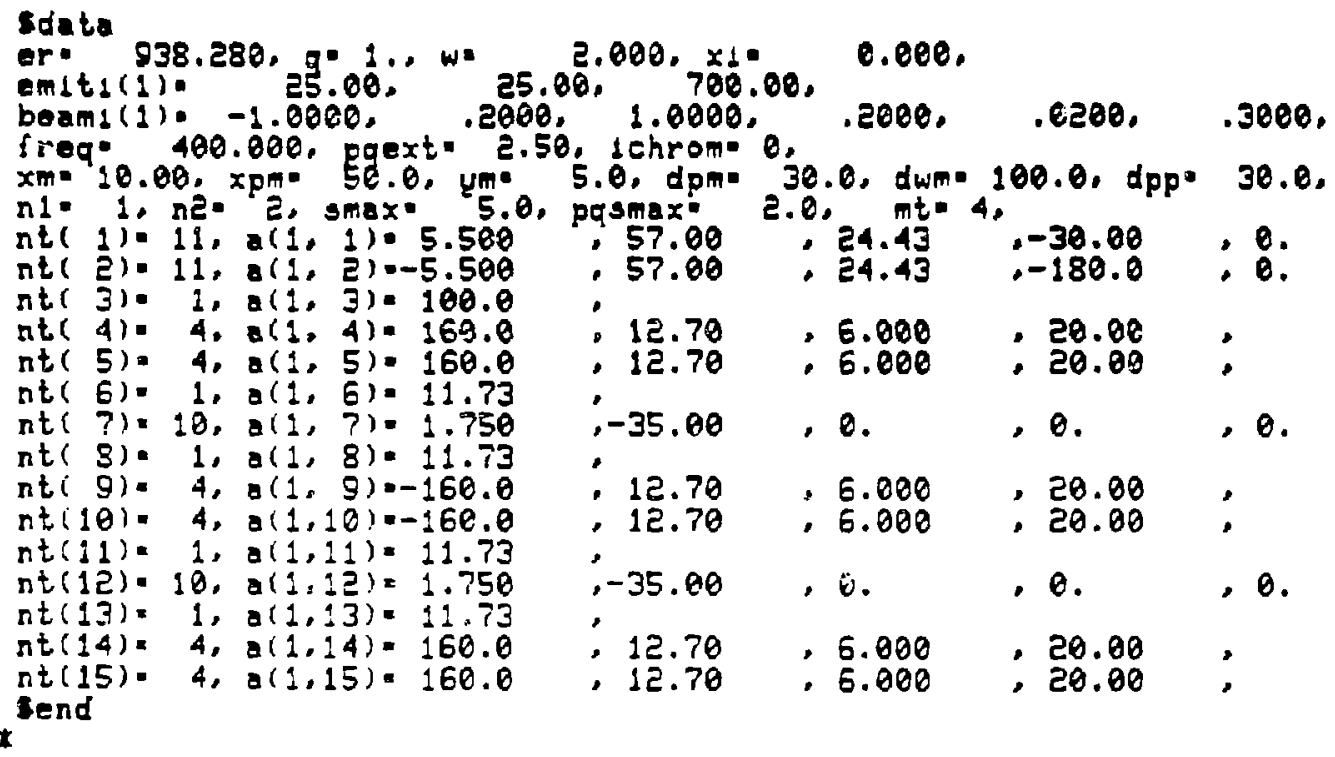

Fig. 1. Input file (to be read as TAPE30) for Example A. 
defines a $2-\mathrm{MeV}$ proton beam with a transverse emittance of $25 \pi \cdot \mathrm{mm} \bullet \mathrm{mrad}$ and a longitudinal emittance of $700 \pi \cdot d e g \cdot k e V$. The transport system consists of the final two cells of a $400-M H z$ RFQ and the first 2 cells of a 400-MHz DTL, separated by a 100-mm drift space. This drift space will later be replaced by drifts, quadrupoles, and an rf gap for transverse and longitudinal matching.

The current of interest is $75 \mathrm{~mA}$ but matching will first be done for $0 \mathrm{~mA}$. If the design of the two structures has made the focusing strengths per unit length about the same in each, a match found for zero current should be acceptable for any current. ${ }^{11}$ Multicurrent matches are desirable particularly when permanent-magnet quadrupoles are used, as they are in this example.

The first step is to determine the matched ellipse parameters at the exit of the RFQ, first for zero current and then for $75 \mathrm{~mA}$. For this purpose, the two RFQ cells must be one period in a focusing system. Values for $V / r_{0}^{2}$ and $A V$ are $5.5 \mathrm{kV} / \mathrm{mm}^{2}$ and $57 \mathrm{kV}$, respectively. Assuming that the horizontal vanes are nearer to the longitudinal axis at the beginning of the first cell, $\mathrm{V} / \mathrm{r}_{\mathrm{O}}^{2}$ is +5.5 for the first cell and -5.5 for the second cell. The length of each cell is $24.43 \mathrm{~mm}$, which is $B \lambda / 2$ for $2-\mathrm{MeV}$ protons at $400 \mathrm{MHz}$. A synchronous phase of $-30^{\circ}$ is assumed, and the fifth parameter in both cells is set to zero to avoid energy changes.

The input file is already set to do matching through the two RFQ cells for zero current. That is, $X I=0, N 1=1, N 2=2$, and $M T=4$. When the " $m "$ command is issued, the program finds the input ellipse parameters that equal the output ellipse parameters in all three phase planes. It is important to remember that none of the alphas or betas should be zero when the matching is begun, because zero values will not be changed.

After the matching procedure, one can see the input and output beam ellipses and the profiles through the RFQ ce'ls by issuing the "g" command. The graphics display is shown in Fig. 2. The results of issuing the "f " $w$ " commands are also shown in this figure. The " $f$ " command determines the phase advances and the matched ellipse parameters calculated from the transfer matrix. The " $w$ " command gives information on the longitudinal properties of the beam, the output phase and energy of the beam center, the half-widths or the phase and energy spreads, the half-widths in $z$ - and $\Delta p / p$, and the emittance in $z-\Delta p / p$ phase space. 


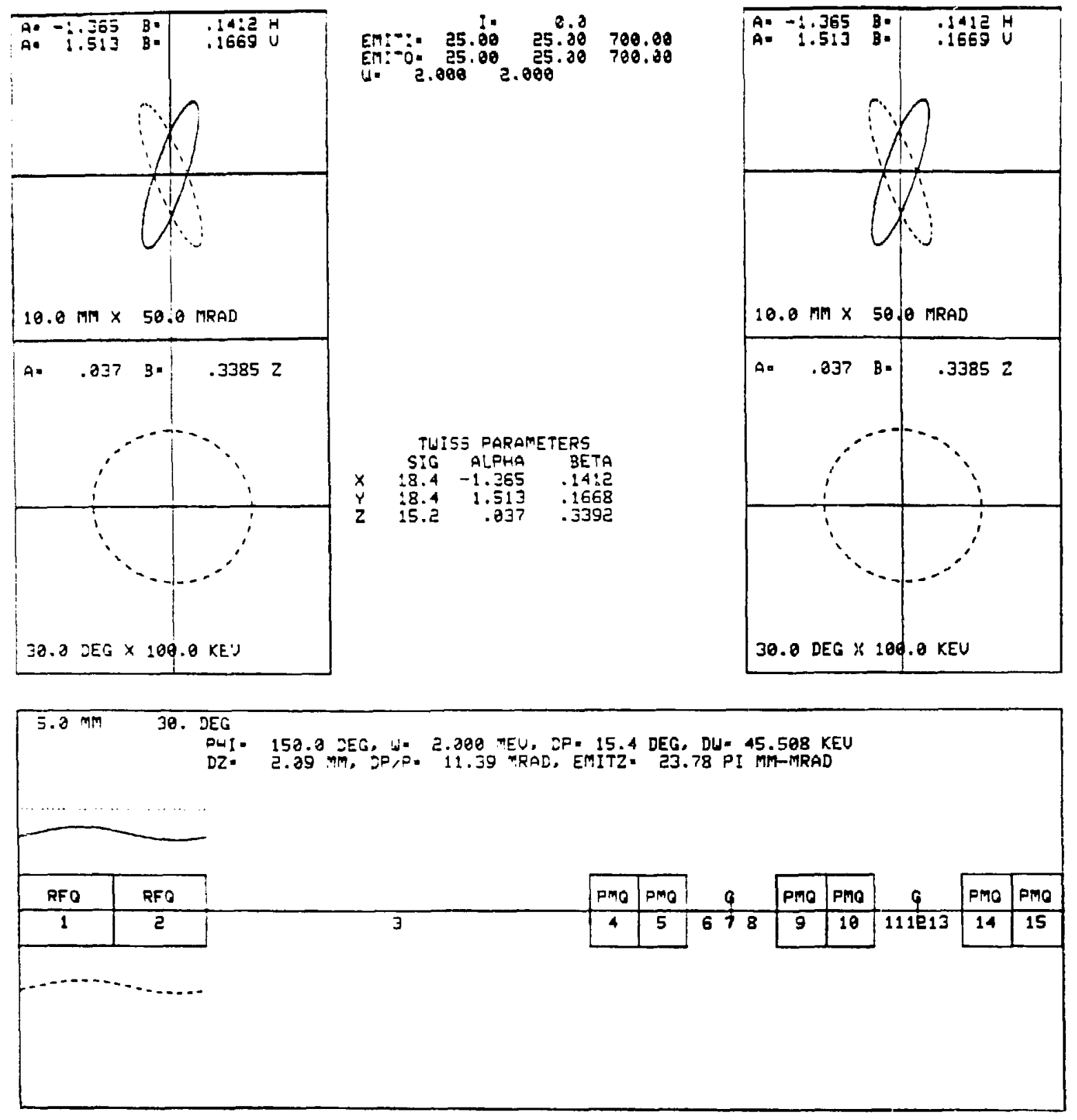

Fig. 2. Graphical display showing the matched output from the RFQ for zero current, the Twiss parameters, and the longitudinal parameters (Example A).

The zero-current ellipse parameters will be saved in the first of five holding arrays by issuing the " $s$ " command and responding to the question about "which beam to save and where" by entering a 1,1. The match for $75 \mathrm{~mA}$ is found by getting into the input mode by typing " $i$ ", changing the current to 
75, and then issuing the "m" command. If the inatching piocess does not converge after the first set of iterations, iepeat the command one or more times. The matched soluti... along with the phase advances and the longitudinal information, is shown in Fig. 3. This solution is saved in the second holding position by again issuing the "s" command and iesponding with a 1,2.

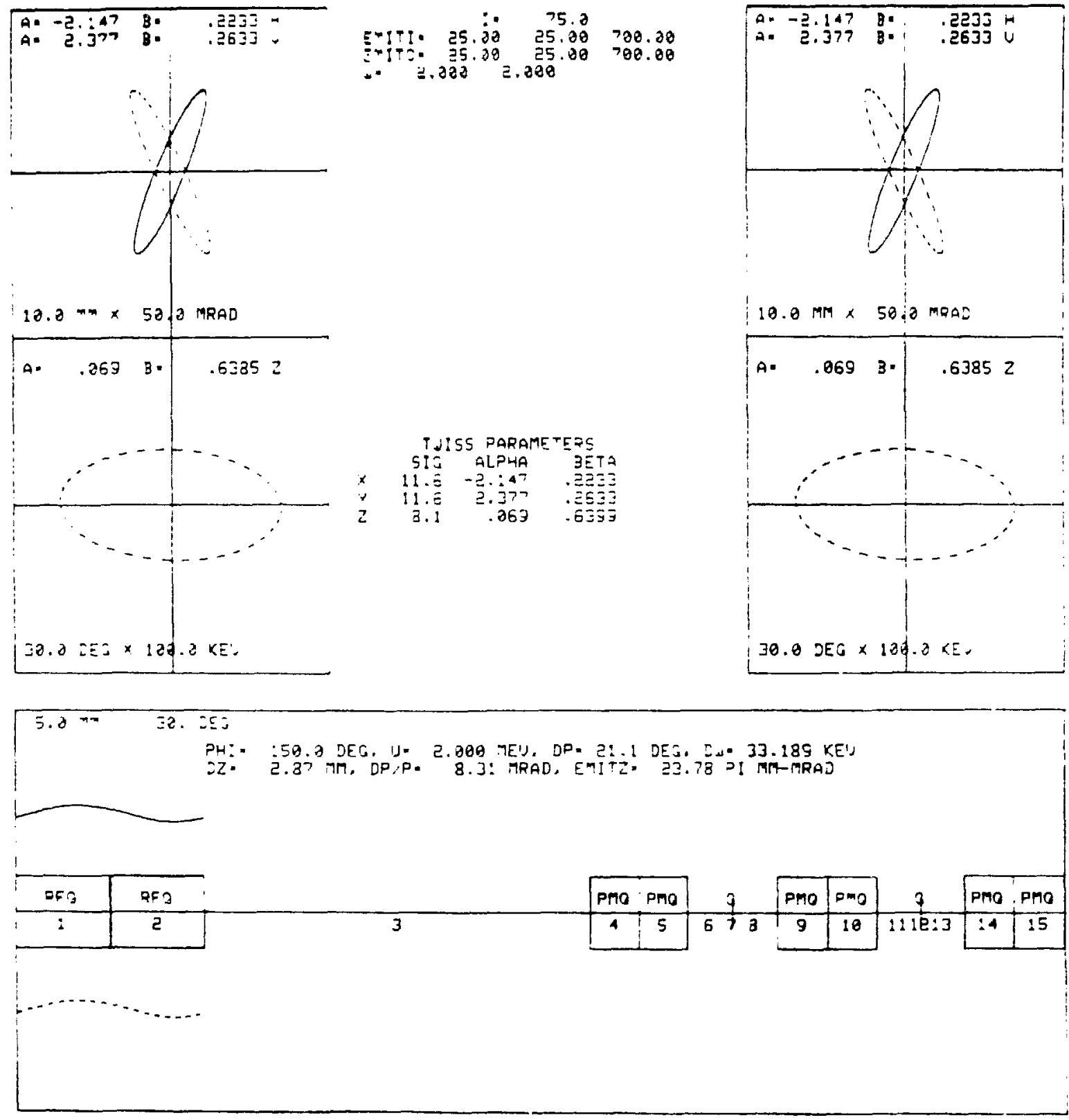

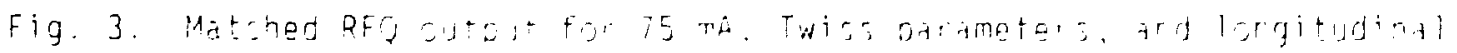

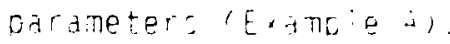


The next step is to find the ellipse parameters that are matchen to the input of the DTL, the first two cells of which are represented by a sequence of permanent-magnet quadrupoles, drifts, and rf-gaps. The transverse focusing period of interest begins in the middle of the first quadrupole and ends in the middle of the third quadrupole. The other halves of the first and third quadrupole must be inclided in the transport system because they affect the magnetic fields in their neighborhoods. The PMQ gradients are $160 \mathrm{~T} / \mathrm{m}$ and their lengths are $25.4 \mathrm{~mm}$. The inner and outer radii of the quadrupoles are 6 and $20 \mathrm{~mm}$, and because PQEXT $=2.5$ in the data file, the fringe fields will extend to $2.5 \times 6 \mathrm{~mm}$. The effective accelerating field strength, $E_{O} T$, in eact: cell is $1.75 \mathrm{MV} / \mathrm{m}$. The length of each cell is $48.86 \mathrm{~mm}$, equal to $\beta \lambda$ for $2 \mathrm{MeV}$-protons at $400 \mathrm{MHz}$. To make the two cells identical, no energy gain is allowed. The synchronous phase is $-35^{\circ}$.

To find the matched ellipse parameters for zero current, get in the input mode and set $X I=0, N 1=5, N 2=14$, and $M T=3$. Then give the "m" command. When the solution is found, issue the " $g$ ", "f", and " $w$ " commands. The result is shown in Fig. 4. Save the solution in the third holding position using the " $s$ " command. Change the current to $75 \mathrm{~mA}$, repeat this procedure (see Fig. 5 for the results), and save the solution in the fourth holding position. Notice that the zero-current transverse phase advances in the two structures are the same per unit length, $18.4^{\circ} / \beta_{\lambda}$. The zero-current longitudinal phase advances are nearly the same, $15.2^{\circ} / \beta \lambda$ for the $R F Q$ and $16^{\circ} / \beta \lambda$ for the DTL. If the quadrupole periodicity is continued upstream of the DTL and an rf-gap is added, the output of the RFQ should be well matched into the DTL both transversely and longitudinally.

We will add the necessary elements and reduce the length of the long drift to a small distance that will change the RFQ output beam to one more nearly round in real space; the shape it should be at the mid point between quadrupoles where our periodic transport will begin.

The elements are inserted after giving the command "a". Figure 6 shows the exact procedure, with a series of input commands to avoid a string longer than 100 characters. Both the type array NT and the element array A must be corrected for the eight new elements. Also included in the figure are the results of a "p", to print the transport system, and a "b", to show the contents of the beam holding positions from our saves during the first matching efforts. 


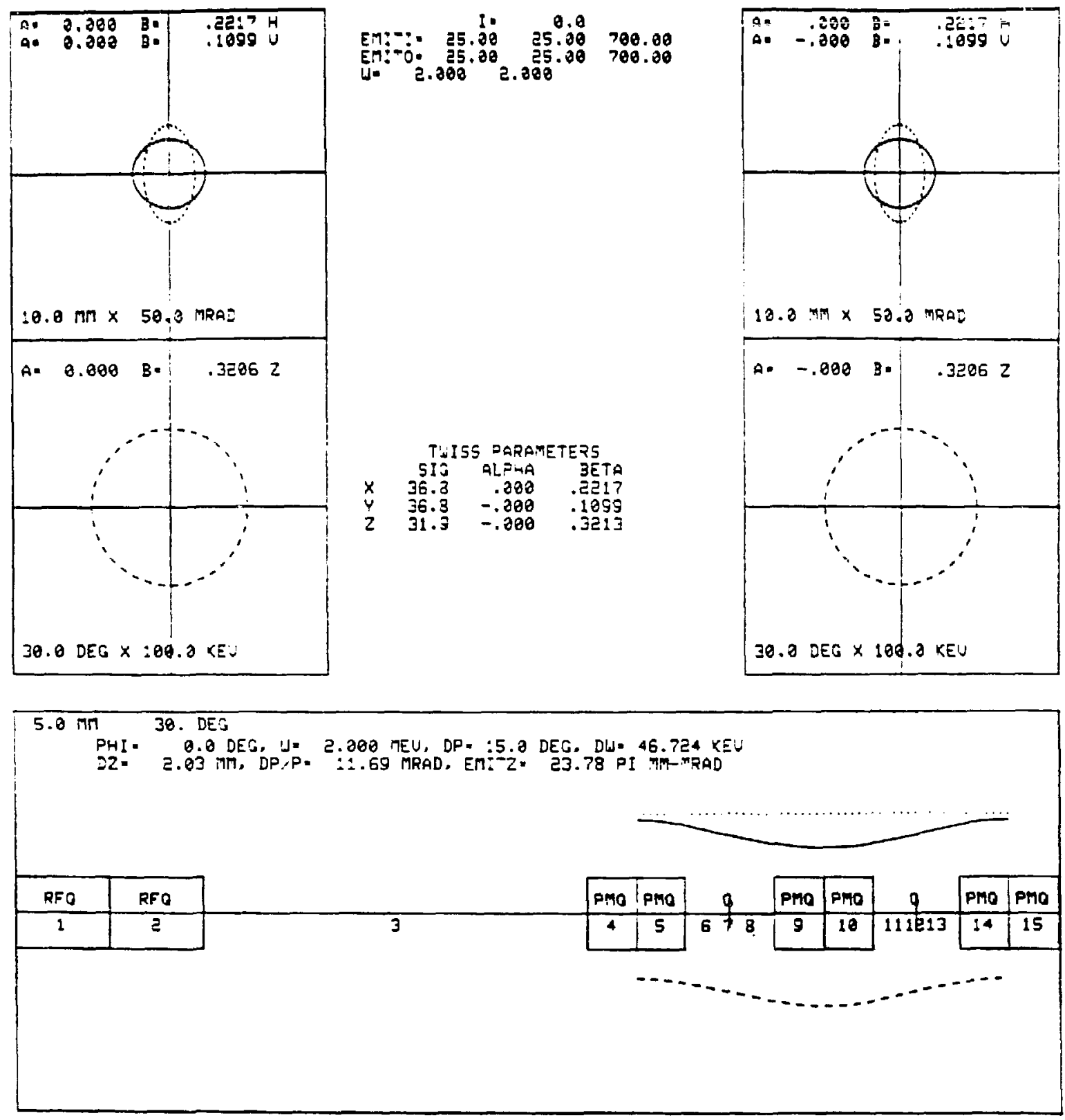

Fig. 4. Matched input to DTL for zero current, Twiss parameters, and longitudinal parameters (Example $A$ ). 

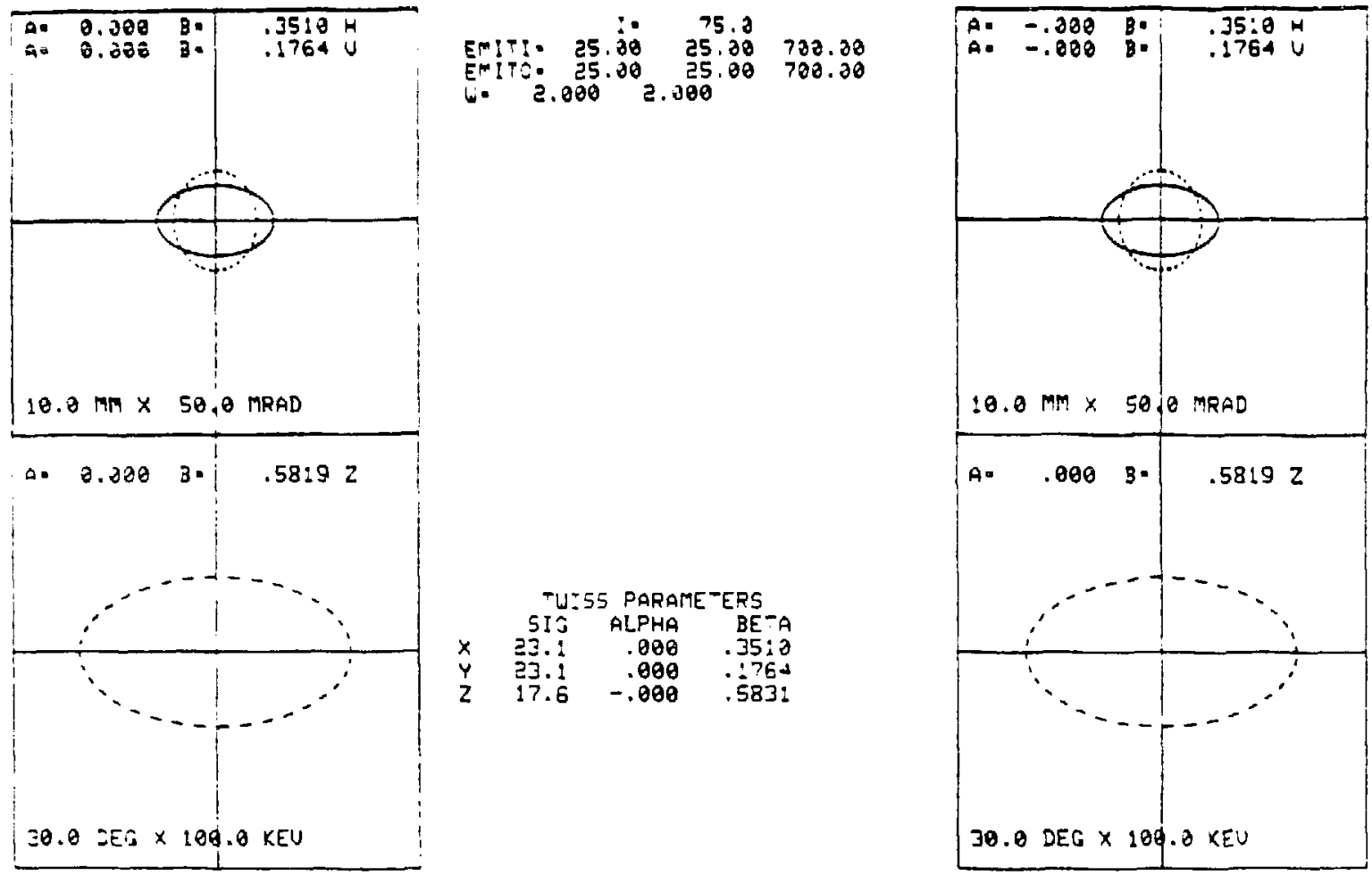

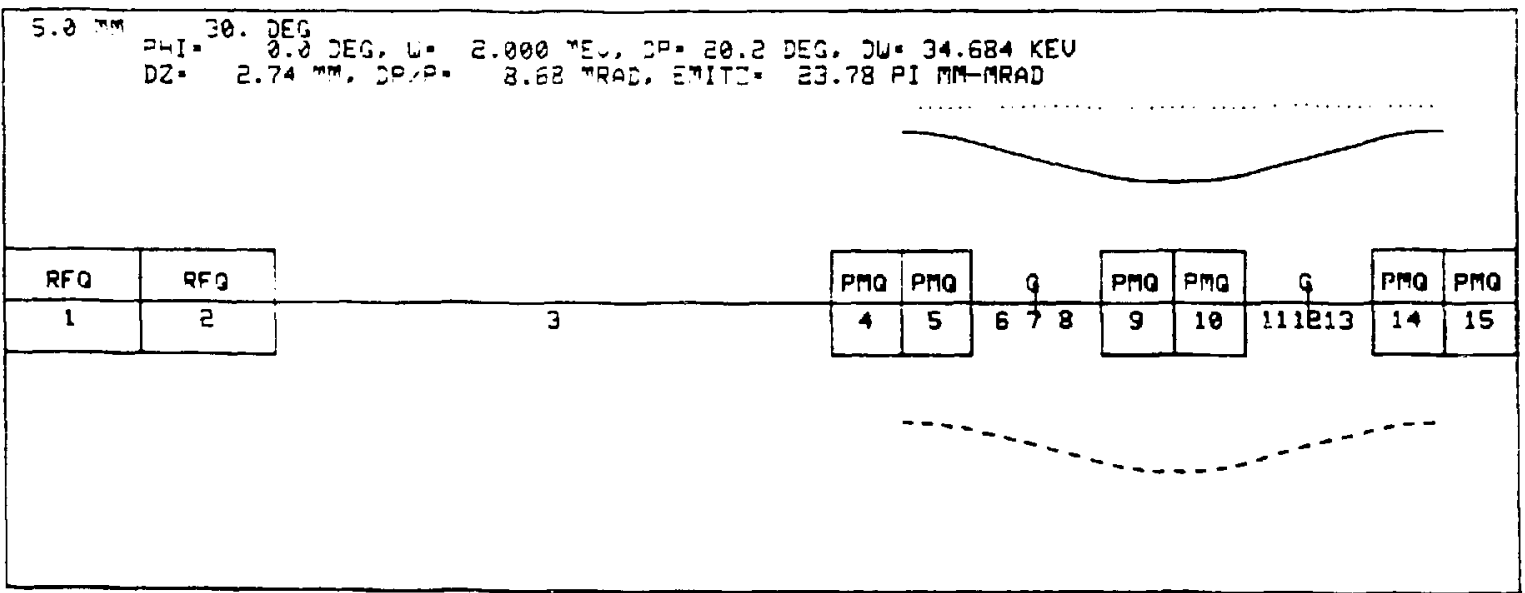

Fig. 5. Matched input to DTL for $75 \mathrm{~mA}$, Twiss parameters, and longitudinal parameters (Example A). 
enter $\mathrm{ml}, \mathrm{m} 2$ (insert $\mathrm{m} 1$ Elements before Element $\mathrm{m} 2$ )

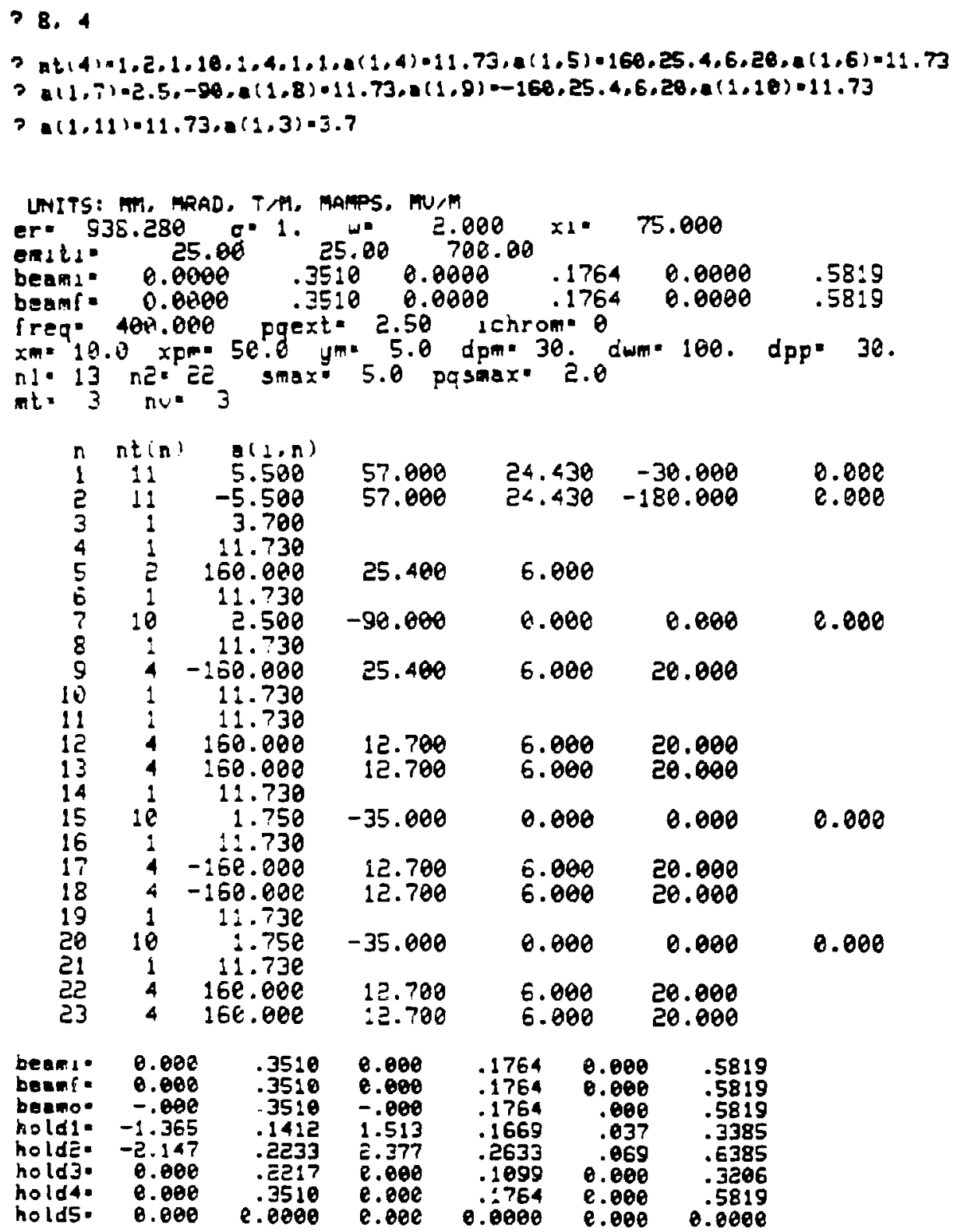

Fig. 6. Procedure for adding eight transport elements before Element 4, a print of the data after the elements have been added, and a print of the beam and holding arrays (Example A). 
Next, the matching section is tested by following the beam from the RFQ to the begirining of the DTL and comparing the beam parameters with those of the matched beam at that position. Two update ("u"; commands must be made. In response to the query about "which beam to restore and whence," a response of "1,1" moves the matched output for the RFQ to BEAMI from holding position 1. Next, the matched beam for the DTL is moved to BEAMF from holding position 3 by responding 2,3. The current is set to zero, and $N 1$ and $N 2$ are set to 1 and 12 after an input command. The command " $g$ " is given, then an " $O$ " is typed to compare the output beam to BEAMF and to print the mismatch factors as shown in Fig. 7. To illustrate another command, the $E_{O}^{\top}$ parameter for Element 7 was changed from 2.5 to $2.3 \mathrm{MV} / \mathrm{m}$, and a " $t$ " caused a second trace on the graph. A repeat of the " $O$ " command shows slight improvement in mismatch factors.

Before repeating the procedure for $75 \mathrm{~mA}$, a printing option on graphics output was initiated by going into input mode and typing "nprin $=4$, ijprin $(1,1)=$ $1,5,1,9,1,7,1,3 "$. Now values for the first parameters of Elements 5 , 9, 7, and 3 will be printed whenever a graph is drawn.

Next, BEAMI and BEAMF were updated from holding positions 2 and 4 , current was changed to $75 \mathrm{~mA}$, a " $\mathrm{g}$ " command was issued, and the mismatch factors were printed as shown in Fig. 8. Notice that the values of four element parameters are now printed.

The mismatch factors, all of which are less than 0.1 , are almost acceptable, but we shall go through a matching procedure to see if they can be improved. We will use Matching Type 9, which expects six variables to be changed. However, if fewer variables are given, matching will still be attempted but an exact solution will not be found. Only four values will be changed in our example: gradients for Elements 5 and 9 , length of drift Element 3 , and $E_{0} T L$ of Gap Element 7. These are set by typing "mt $=9$, $m p(1,1)=1,5,1,9,1,7,1,3$ " in input mode. Successive typing of " $m$ " until there was no further decrease in largest mismatch factor gave the results shown in Fig. 9. Because the optional printed parameters are the same as the matching variables, the final selected values can be easily seen. With all mismatch factors below 0.05 , a good solution has been obtained. 


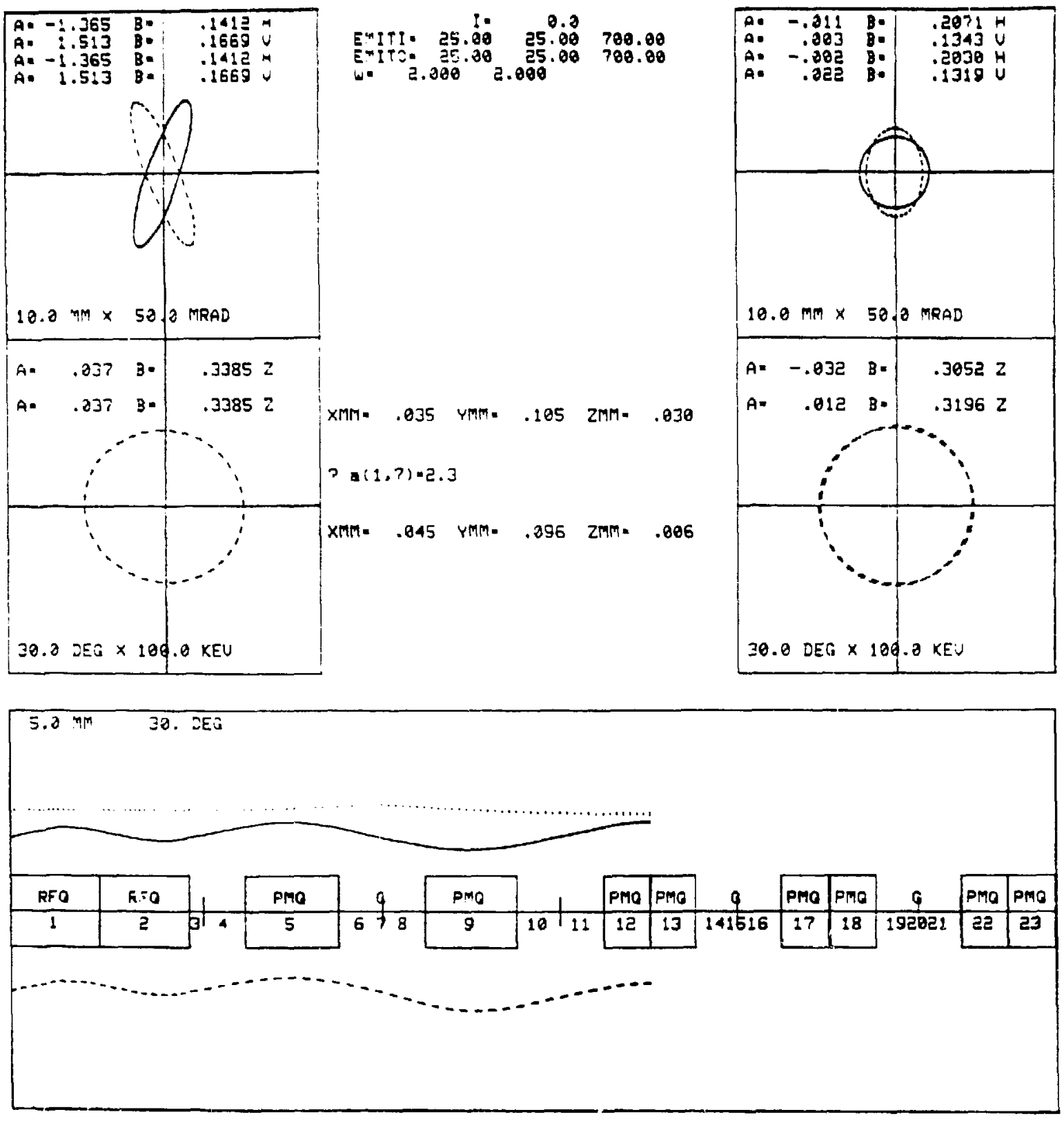

Fig. 7. Matched RFQ output for zero current followed to input of DTL. Run repeated for $\mathrm{rf}$-gap field changed to $2.3 \mathrm{MV} / \mathrm{m}$ (Example A). 


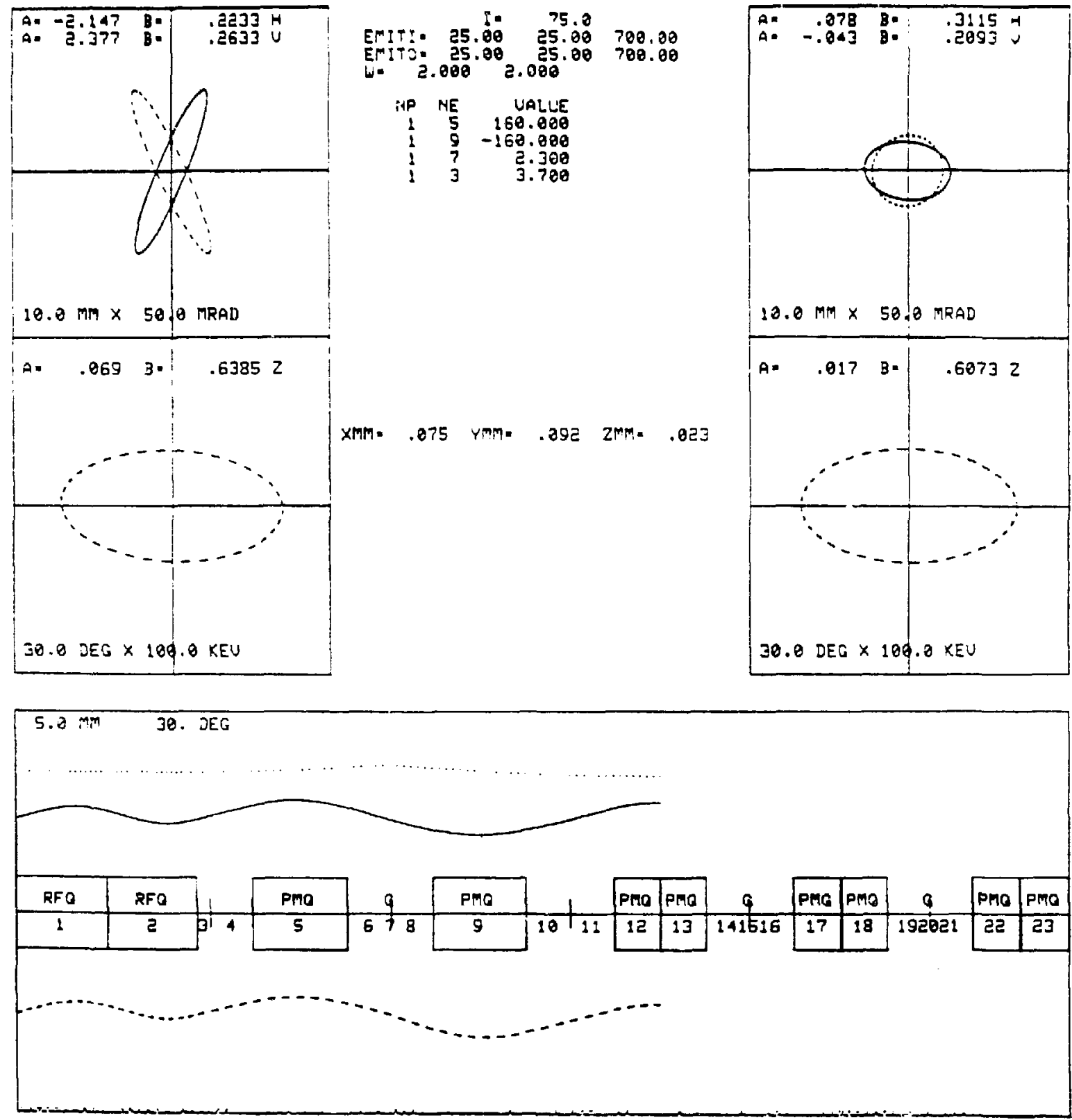

Fig. 8. Matched RFQ output for $75 \mathrm{~mA}$ followed to input of DTL (Example A). 


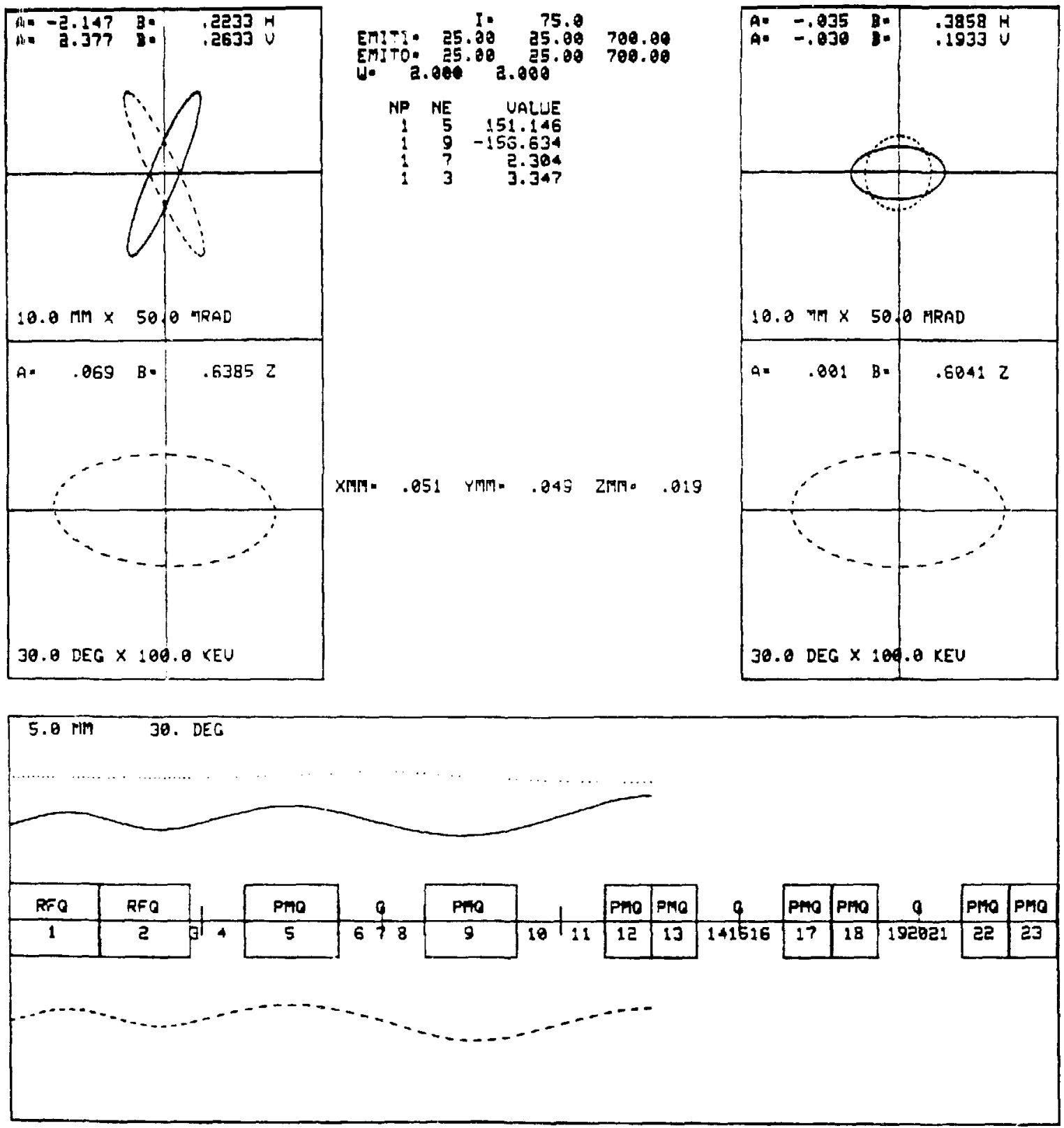

Fig. 9. Matched RFQ output for $75 \mathrm{~mA}$ followed to input of DTL after the first parameter in Elements 3, 5, 7, and 9 have been adjusted by the matching procedure (Example A). 


\section{B. Matching Between 80-MHz RFQ and DTL}

A different approach to matching between an RFQ and DTL must be used either when the periodicity of the quadrupoles is not maintained or when the two structures have different focusing strengths. No buncher or rf gap will be added, so longitudinal matching will be ignored. Transverse matching will be accomplished by using the first four quadrupoles of the DIL as matching variables. It is not expected that the solution will be appropriate for other currents or emittances.

In our example, the frequency is $80 \mathrm{MHz}$, the energy is $2 \mathrm{MeV}$, the 100-mA deuteron beam has a transverse emittance of $60 \pi \cdot \mathrm{mm} \bullet \mathrm{mrad}$ and a longitudinal emittance of $1000 \pi \cdot k e V \cdot d e g$. The initial step (not shown) is to find the matched beam for the final two cells of the RFQ and save the beam as we did in the first example.

Next, also not shown, we simulate (with electromagnetic quadrupoles, drifts, and $r f$ gaps) two cells of a periodic DTL with no energy gain. Instead of the conditions at the beginning of the DTL, we select those at the end of Cell 4 and match into a periodic structure at that point. The energy is $2.594 \mathrm{MeV}, B \lambda$ is $196.7 \mathrm{~mm}, E_{O} T$ is $1.05 \mathrm{MV} / \mathrm{m}$, and the $96-\mathrm{mm}$ quadrupoles have a gradient of $26.2 \mathrm{~T} / \mathrm{m}$. The beam energy $W$ must be changed to 2.594 , and the unnormalized transverse emittances reduced from 60 to $52.69 \pi \cdot \mathrm{mm} \cdot \mathrm{mrad}$. The longitudinal emittance EMITI( 3 ) does not have to be changed. A matched beam for these two cells is determined and saved as in the first example.

Next, the two RFQ cells, a drift, and the first four cells of the DTL are made into a transport system as shown in Fig. 10. Notice that the initial energy is $2 \mathrm{MeV}$ and that each $\mathrm{rf}$ gap element, Type 10, has an energy increase as the fourth parameter. Each cell has been given the correct total iength, but the rf gap is not positioned off-center as it more accurately could be. BEAMI has the correct values for matched input to the RFQ, BEAMF has the values of the matched beam at the end of Cell 4, or Element 20, and will be used to calculate mismatch factors. The drift space between the RFQ and DTL is about one $B \lambda$, a distance long enough to allow separation of the structures, but is short enough to prevent extreme debunching of the beam. The file has already been prepared for matching by entering the proper parameter and element numbers into the MP array (Parameter 1 of Elements 4, 8 , 12, 16) and setting $M T=8$ and $N V=4$. (NV is not set automatically until 


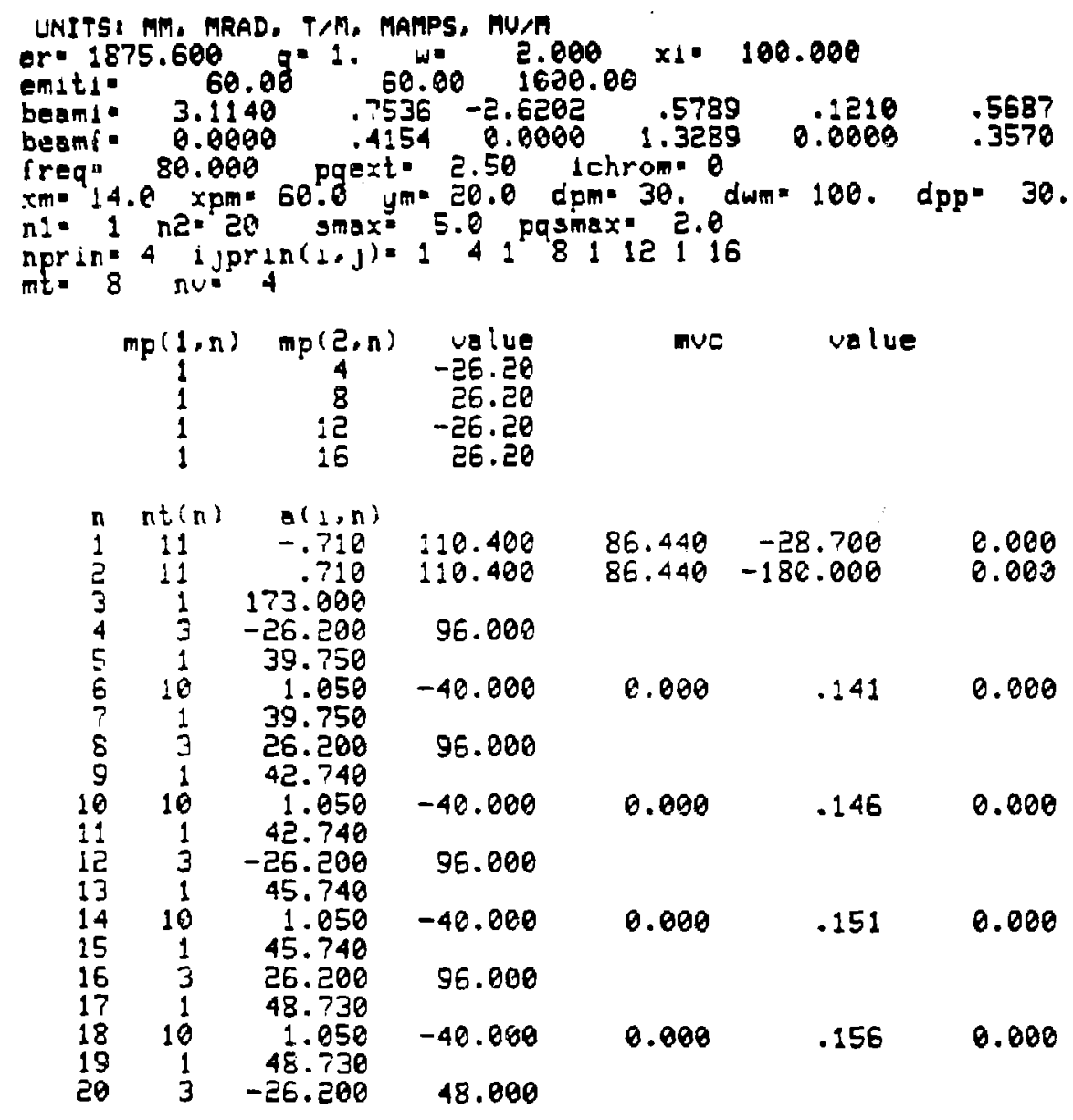

Fig. 10. Result of the "p" (print) command for the initial conditions of Example B.

matching is requested, but it can be input at any time so the variables will be piinted.) Before matching, the input beam is followed through all the elements and the mismatch factors printed ( $F i g .11$ ).

A match is now made by typing "m" until convergence is obtained. The results are displayed by typing " $g$ " and "O" as shown in Fig. 12. The new quadrupole values, which are printed because of the previous setting of the NPRIN and IJPRIN variables, are reasonable. The transverse solution is exact. Notice the increase in energy as shown by " $W=2.0002 .594$ " and the apparent decrease in transverse emittances. There is, of course, no change in normalized emittance; the values printed are unnormalized. 

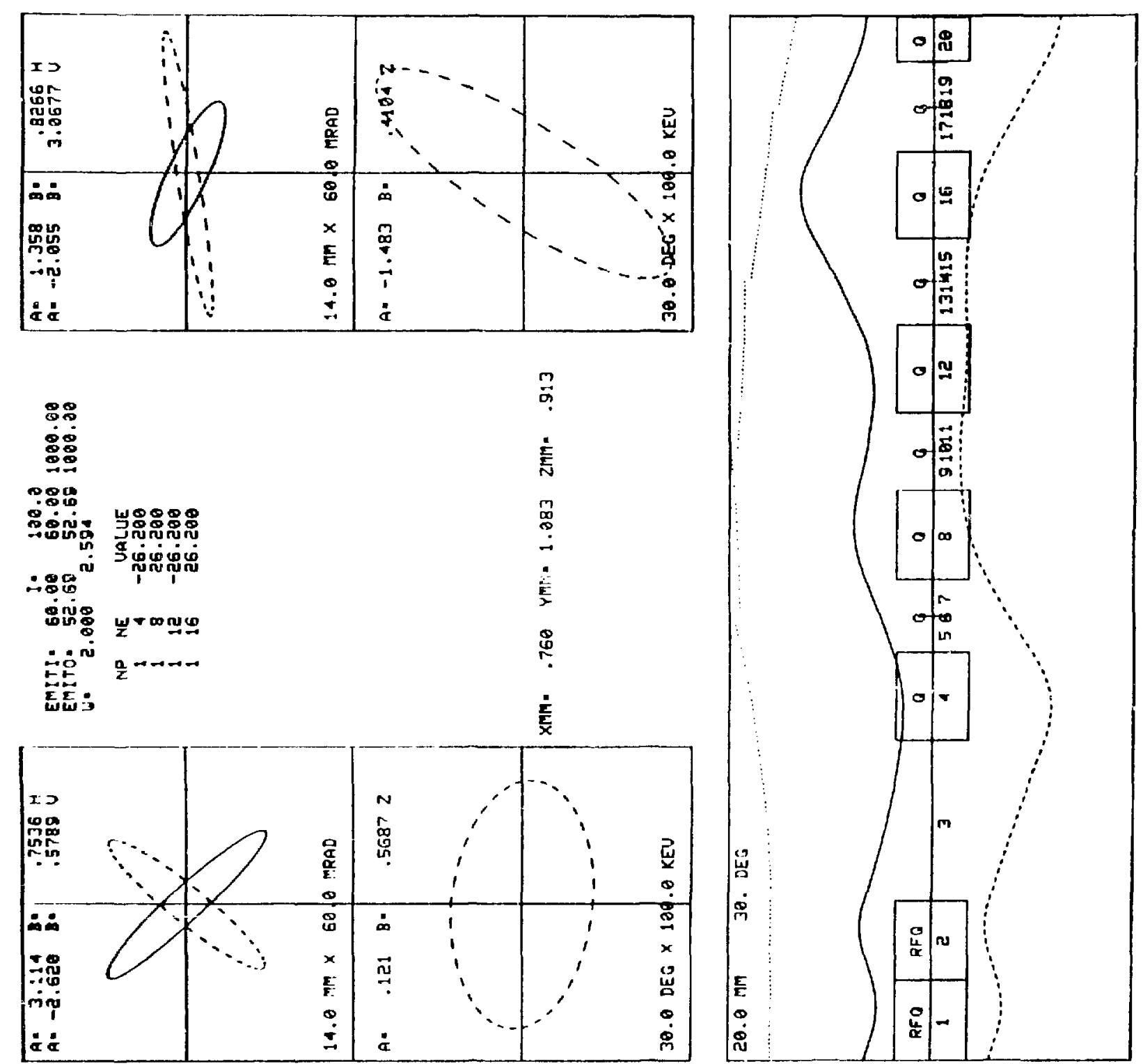

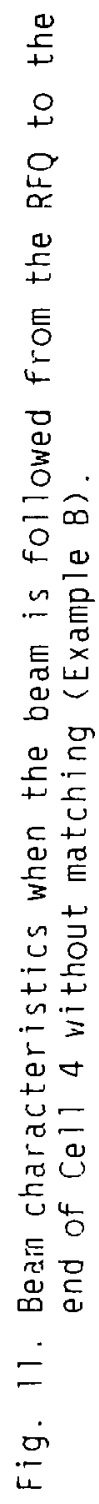

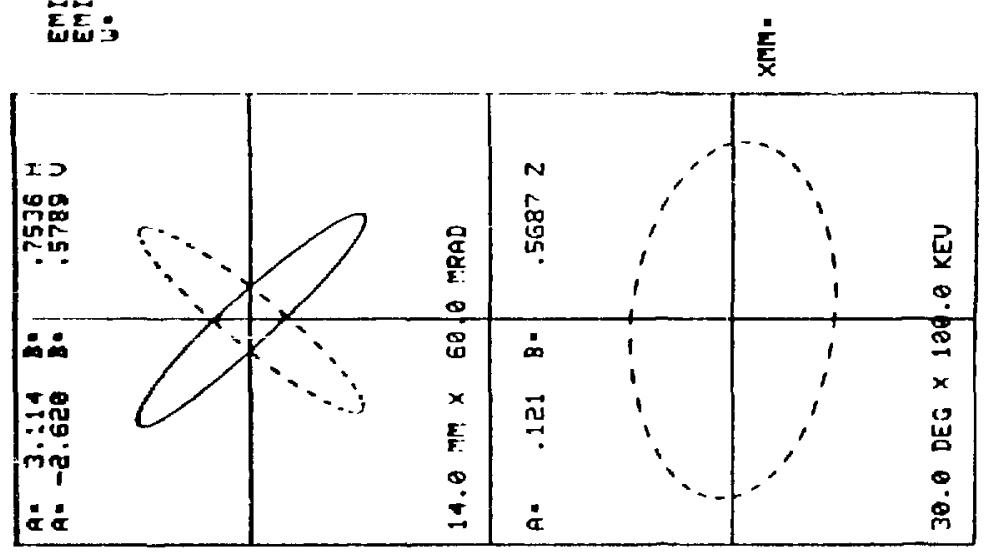




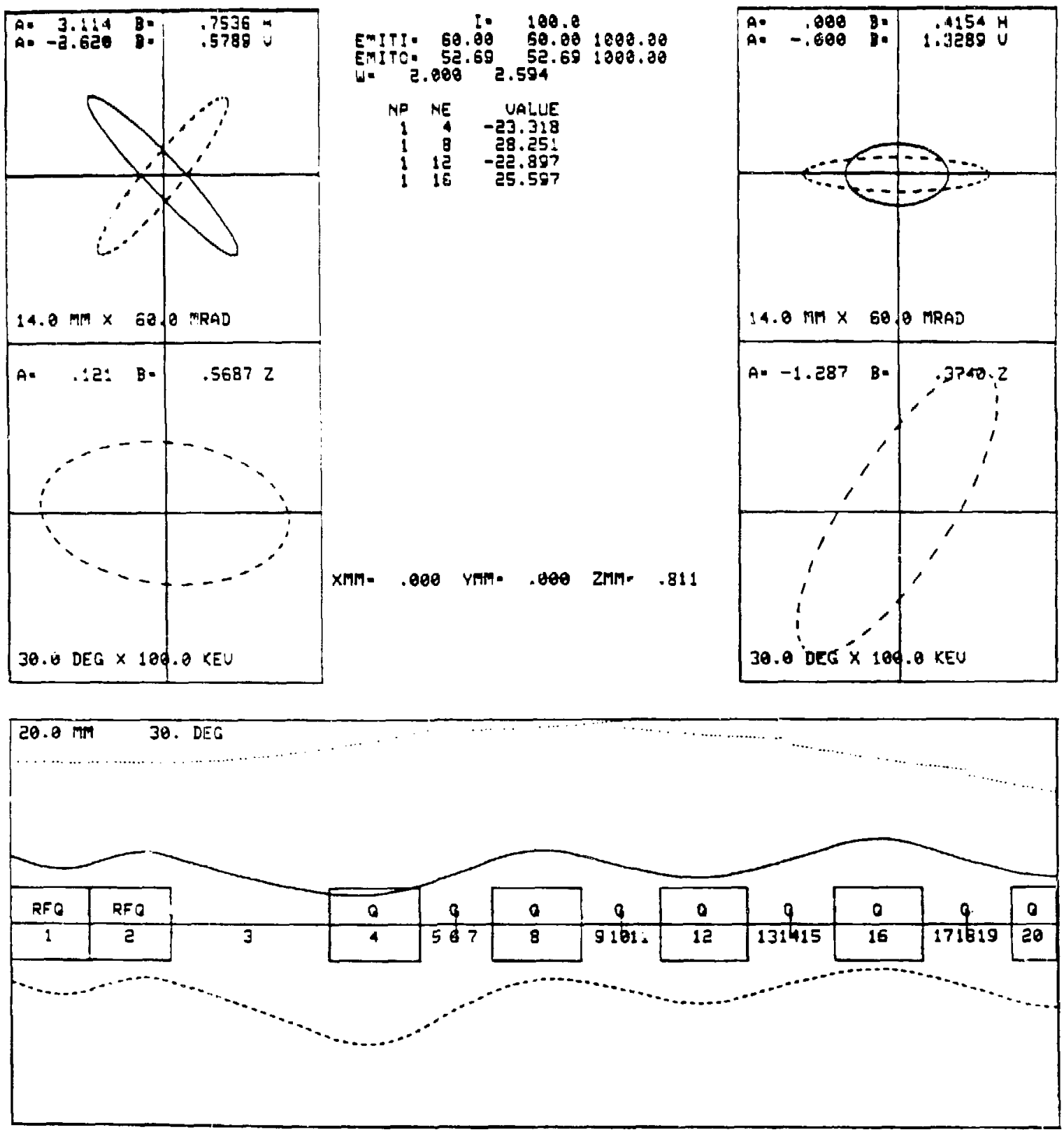

Fig. 12. Beam characteristics in Example B after quadrupole gradients in Elements $4,8,12$, and 16 have been adjusted by the matching procedure (Example $B$ ). 


\section{Electron Linac and Transport System}

This example shows the transport of an electron beam through triplets, a linac tank, and some bending magnets. Figure 13 shows the data printed by issuing the "p" command, and Fig. 14 shows the graphics display produced when the " $\mathrm{g}$ " command is given. A $1000-\mathrm{mA}, 10-\mathrm{MeV}$ electron beam is passed through a triplet and then accelerated to about $20 \mathrm{MeV}$ by a 15 -cell, 1300-MHz linac tank. Because of the increased energy, the unnormalized transverse emittance is reduced from $6 \pi \bullet \mathrm{mm} \bullet \mathrm{rad}$ to $3.06 \pi \bullet \mathrm{cm} \bullet \mathrm{rad}$. After it passes through the tank, the beam then passes through a quadrupole triplet, three bending magnets, and another triplet. In the absence of space-charge forces, the three bending magnet; would be an achromatic system. The space-charge forces prevent the system from being completely achromatic, and a small change is seen in the emittance in the horizontal and longitudinal planes.

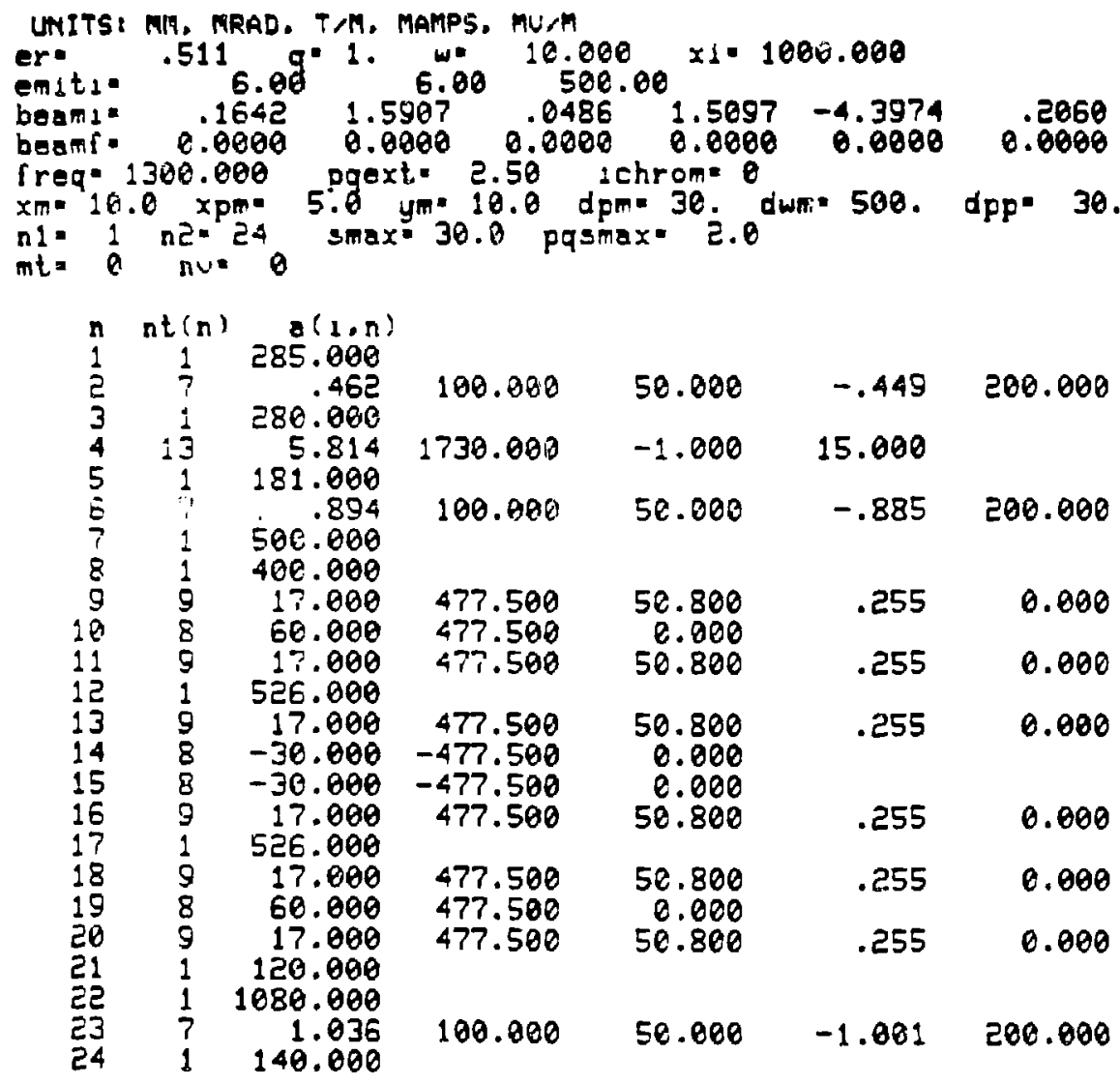

Fig. 13. Results produced by "p" command for transport system of Example $C$. 

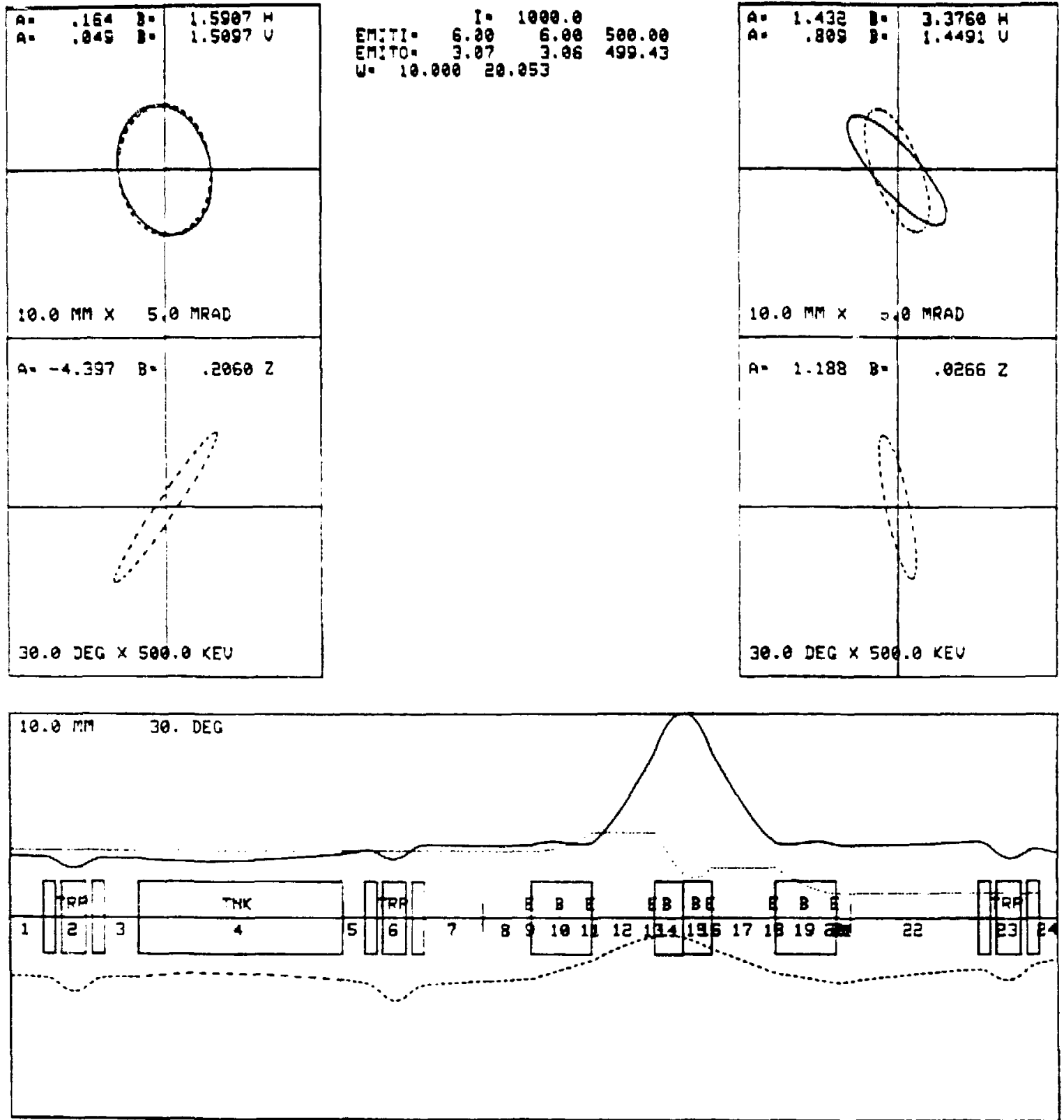

Fig. 14. Beam characteristics for Example $C$.

ACKNOWLEDGMENT

R. 5. Mills helped with the coding, contributed many good ideas, and wrote most of sec. Ti for this report. Her contributions are gratefully acknowledged. 


\section{APPENDIX A}

THE O-MATRIX

The general equation for an n-dimensional ellipsoid may be written as

$\sum_{i=1}^{n} \sum_{j=1}^{n} a_{i j} u_{i} u_{j}=1$,

where $u_{k}$ denotes distance along the $k$ th coordinate axis. (In TRACE 3-D, $n$ is 6 and the coordinates are $x, x^{\prime}, y, y^{\prime}, z$ and $\Delta \rho / \rho$.) There are two coefficients for each $u_{i} u_{j}$ product when $i \neq j$, namely $a_{i j}$ and $a_{j i}$. Because only the sum $a_{i j}+a_{j i}$ is important, we can define $a_{i j}=a_{j i}$ without losing any generality and express the ellipsoid equation in matrix form. Let $U$ be the column vector of the coordinates,

$U=\left\{\begin{array}{l}u_{1} \\ u_{2} \\ u_{n}\end{array}\right\}$,

and $\sigma^{-1}$ represent the symmetric coefficient matrix,

$\sigma^{-1}=\left[\begin{array}{llll}a_{11} & a_{12} & \cdots & a_{1 n} \\ a_{21} & a_{22} & \cdots & a_{2 n} \\ a_{n 1} & a_{n 2} & \ldots & a_{n n}\end{array}\right]$

The reason for defining this coefficient matrix to be $\sigma^{-1}$ rather than $\sigma$ will become apparent later.

The ellipsoid equation in matrix form is

$U^{\top} \sigma^{-1} U=1$

where $U^{\top}$ is the transpose of $U$. For this to represent an n-dimensional ellipsoid, all of the diagonal elements of the $\sigma^{-1}$-matrix must be positive. 
Let $\sigma_{1}^{-1}$ represent a matrix that defines a particular ellipsoid, and let $U_{1}$ be any point on the surface of the ellipsoid. Then

$$
U_{1}^{\top} \sigma_{1}^{-1} U_{1}=1
$$

Let $R$ be the transfer matrix that transforms point $U_{1}$ to point $U_{2}$ :

$$
\mathrm{U}_{2}=\mathrm{RU}_{1}
$$

The point $U_{2}$ will lie on the surface of another ellipsoid having the coefficient matrix $\sigma_{2}^{-1}$. The following matrix manipulations show how to obtain $\sigma_{2}^{-1}$ from $\sigma_{1}^{-1}$ and $R$.

$$
\begin{aligned}
& U_{1}^{\top} \sigma_{1}^{-1} U_{1}=1, \\
& U_{1}^{\top} R^{\top}\left(R^{\top}\right)^{-1} \sigma_{1}^{-1} R^{-1} R U_{1}=1,
\end{aligned}
$$

and

$$
\begin{aligned}
& \left(R U_{1}\right)^{\top}\left(R^{\top}\right)^{-1} \sigma_{1}^{-1} R^{-1}\left(R U_{1}\right)=1 . \\
& U_{2}^{\top}\left(R \sigma_{1} R^{\top}\right)^{-1} U_{2}=1 .
\end{aligned}
$$

This is the equation for the second ellipsoid, with

$$
\left.\sigma_{2}^{-1}=\left(R \sigma r_{1}\right)^{\top}\right)^{-1}
$$

or

$$
\sigma_{2}=R \sigma_{1} R^{\top}
$$

Meaning of o-Matrix Elements

Define a function

$$
f(U)=U^{\top} o^{-1} U
$$

The condition $f(U)=$ constant defines the surface of a hyperellipsoid (if all diagonal elements of $\sigma^{-1}$ are positive) and $f(U)=1$ defines the surface of

58 
the particular hyperellipsoid discussed above. At any point $U$, the gradient of $f(U)$ is a vector normal to the surface of the hyperellipsoid passing through U.

The gradient in $n$-dimensional space is defined as

$\nabla f=\sum_{k=1}^{n} \frac{\partial f}{\partial u_{k}} \hat{u}_{k}$,

where $\hat{u}_{k}$ is the unit vector along the kth coordinate axis. Writing $f(U)$ in terms of the matrix elements.

$f(u)=\sum_{j=1}^{n} \sum_{i=1}^{n} a_{i j} u_{i} u_{j}$,

the gradient is

$\nabla f(u)=\sum_{k=1}^{n}\left[\sum_{j=1}^{n} a_{k j} u_{j}+\sum_{i=1}^{n} a_{i k} u_{i}\right] \hat{u}_{k}$.

Because $a_{i j}=a_{j i}$, and $\hat{u}_{k}$ is a column vector whose only nonzero element is a 1 in the kth row, the gradient of $f$ can be expressed in matrix notation:

$\nabla f(U)=2 \sigma^{-1} U$.

At the point $U_{k}$ on the surface at which the $k$ th coordinate has its maximum extent, the gradient is parallel to $\hat{u}_{k}$ and has a magnitude (unknown) of $\left|\nabla f\left(U_{k}\right)\right|$. Then

$\sigma^{-1} U_{k}=\frac{1}{2}\left|\nabla f\left(U_{k}\right)\right| \hat{u}_{k}$

from which

$U_{k}=\frac{1}{2}\left|\nabla f\left(U_{k}\right)\right| \sigma \hat{u}_{k}$

and

$U_{k}^{\top}=\frac{1}{2}\left|\nabla f\left(U_{k}\right)\right|\left(\sigma \hat{u}_{k}\right)^{\top}$. 
For the hyperellipsoid for which $f(U)=1$,

$$
\begin{aligned}
1 & =U_{k}^{T} \sigma^{-1} U_{k} \\
& =\frac{1}{4}\left|\nabla f\left(U_{k}\right)\right|^{2} \tilde{u}_{k}^{T} \sigma \hat{u}_{k} \\
& =\frac{1}{4}\left|\nabla f\left(U_{k}\right)\right|^{2} \sigma_{k k},
\end{aligned}
$$

where $\sigma_{k k}$ is the $k$ th diagonal element of the $\sigma$-matrix. The magnitude of the gradient at $U_{k}$ is

$\left|\nabla f\left(u_{k}\right)\right|=2 / \sqrt{\sigma_{k k}}$

and the point on the surface at the maximum extent in the kth direction is

$U_{k}=\sigma \quad \tilde{u}_{k} / \sqrt{\sigma_{k k}}$

The term $U_{k}$ is the $k$ th column (and, because $\sigma$ is symmetric, the kth row) of the o-matrix divided by $\sqrt{\sigma_{k k}}$. In particular, $\sigma_{k k}$ is the square of the maximum extent of the ellipsoid in the kth direction, and $\sigma_{k i} / \sqrt{\sigma_{k k}}$ (and $\sigma_{i k}\left(\sqrt{\sigma_{k k}}\right)$ is the value of the $i$ th coordinate at the maximum extent in the $k$ th direction. Each off-diagonal element is therefore related to two diagonal elements, and sometimes they are written as

$\sigma_{i k}=\sigma_{k i}=r_{i k} \sqrt{\sigma_{i j}} \sqrt{\sigma_{k k}}$,

where $r_{i k}$ is referred to as the correlation coefficient. 


\section{APPENDIX B}

TWISS PARAMETERS AND THEIR RELATIONSHIPS TO G-MATRIX ELEMENTS.

The ellipse equation often used in beam-dynamics calculations is

$y x^{2}+2 \alpha x x^{\prime}+\beta\left(x^{\prime}\right)^{2}=\varepsilon$,

where $\varepsilon=E / \pi$, the emittance (area of the ellipse) divided by $\pi$, and $B$ and $Y$ are positive quantities. In this form, $\alpha, \beta$, and $\gamma$ are called the Twiss, or Courant-Snyder, parameters and are related by

$B_{Y}-\alpha^{2}=1$.

The ellipse intersects the positive $x$-axis at $x_{i}=\sqrt{\varepsilon / y}$ and the positive $x^{\prime}-a x$ is at $x_{i}^{\prime}=\sqrt{\varepsilon / B}$, as sketched below.

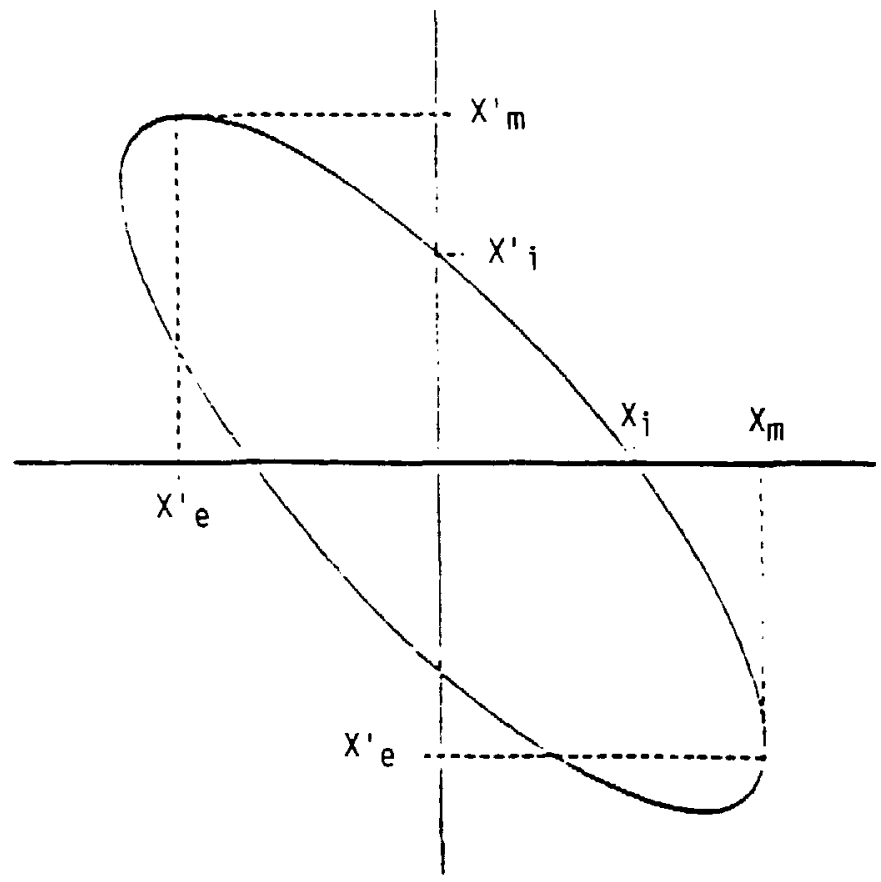

The product of $x_{j}$ and $x_{m}^{\prime}$, the maximum extent of the ellipse in the $x^{\prime}-$ direction, is equal to $\varepsilon$, so $x_{m}^{\prime}=\sqrt{\gamma \varepsilon}$. Likewise, $x_{m}=\sqrt{\beta \varepsilon}$. At the point on the ellipse at which $x^{\prime}=x_{m}^{\prime}$, the $x$-coordinate is $x_{e}=-\alpha \sqrt{\varepsilon / y}$, and at $x=x_{m}, x_{e}^{\prime}=-\alpha \sqrt{\varepsilon / \beta}$. Therefore,

$\alpha=-\frac{x_{e}}{x_{i}}=-\frac{x_{e}^{\prime}}{x_{i}^{1}}$.

For the ellipse shown above, $\alpha$ is positive. 


\section{Relationship Between $\sigma$-Matrix and Twiss Parameters}

The $6 \times 6$ o-matrix can be partitioned into nine $2 \times 2$ submatrices:

$\sigma=\left[\begin{array}{c:c:c}\sigma_{x x} & \sigma_{x y} & \sigma_{x z} \\ \hdashline \sigma_{y x} & \sigma_{y y} & \sigma_{y z} \\ \hdashline \sigma_{z x} & \sigma_{z y} & \sigma_{z z}\end{array}\right]$

The $\sigma_{x x}, \sigma_{y y}$, and $\sigma_{z z}$ submatrices are related to the Twiss parameters in the $x-x^{\prime}, y-y^{\prime}$, and $z-\Delta p / p$ planes, respectively. In App. A, it was shown that the six diagonal elements of the $\sigma$-matrix are the squares of the maximum extent of the ellipsoid in each of the six directions and that $\sigma_{i j}$ is the product of the maximum extent in the $j$ th direction and the value of the $i$ th coordinate at that maximum extent. Then

$\sigma_{x x}=\left[\begin{array}{cc}x_{m}^{2} & x_{e} x_{m}^{\prime} \\ x_{e}^{\prime} x_{m} & \left(x_{m}^{\prime}\right)^{2}\end{array}\right]$.

Using the relationships derived above for $\alpha, B$, and $\gamma$,

$\sigma_{x x}=\left[\begin{array}{cc}B_{x} \varepsilon_{x} & -\alpha_{x}^{x_{i} x_{m}^{\prime}} \\ -\alpha_{x} x_{i} x_{m} & y_{x} \varepsilon_{x}\end{array}\right]$,

where the subscript $x$ has been appended to $\alpha, \beta, \gamma$, and $\varepsilon$ to denote properties on the $x-x^{\prime}$ plane. Because $x_{i} x_{m}^{\prime}=x_{i}^{\prime} x_{m}=\varepsilon_{x}$, this reduces to

$\sigma_{x x}=\left[\begin{array}{cc}\beta_{x} \varepsilon_{x} & -\alpha_{x} \varepsilon_{x} \\ -\alpha_{x} \varepsilon_{x} & \gamma_{x} \varepsilon_{x}\end{array}\right]$

having a determinant

$$
\begin{aligned}
\operatorname{det}\left(\sigma_{x x}\right) & =\left(\beta_{x} \gamma_{x}-\alpha_{x}^{2}\right) \varepsilon_{x}^{2} \\
& =\varepsilon_{x}^{2} .
\end{aligned}
$$




\section{APPENDIX C}

\section{MISMATCH FACTOR}

As the name implies, the mismatch factor is a quantitative measure of the difference between two ellipses having the same area and the same center. If the two ellipses are not identical, one ellipse could he enlarged so that it just encloses the other ellipse; the amount of enlargement is related to the mi smatch factor.

One way of looking at the problem is as follows (See Fig. C-1): First, find the transformation that maps one of the ellipses into a circle, and apply the same transformation to the second ellipse to get a modified ellipse. Denote the radius of the circle by $R_{C}$ and the length of the major semiaxis of the ellipse by $R_{E}$. Because the ellipse and the circle have the same area, $R_{E}$ will be greater than or equal to $R_{C}$. The mismatch factor used in TRACE is

$M=\frac{R_{E}}{R_{C}}-1$.

Periodic beam-transfort systems have matched conditions if they are stable. That is, if one matches a beam to the transport system, and if one could measure the size of the beam at the same location in each period of the system, then one would see a constant beam size. A mismatched beam would oscillate about this matched size, and at some places the beam would be larger by a factor of $1+M$. For example, a mismatch factor of 0.1 , defined as above, would mean that the beam would occasionally be $10 \%$ larger than it would be if it were matched. An example of two ellipses having a mismatch factor of 0.1 is shown in Fig. $\mathrm{C}-1$.

A wo d of caution is in order. It is quite common in the literature for the mismatch factor to be defined as $\left(R_{E} / R_{C}\right)^{2}-1$, which relates to how much the area of the circle would have to be increased to enclose the ellipse. If this definition is used, one would calculate a mismatch factor of 0.21 for the example given above.

The mismatch factor (as defined above) between the two ellipses

$y x^{2}+2 \alpha x x^{\prime}+\beta\left(x^{\prime}\right)^{2}=\varepsilon$ 
and

$G x^{2}+2 A x x^{\prime}+B\left(x^{\prime}\right)^{2}=\varepsilon$

is given by

$M=\left[\frac{1}{2} R+\sqrt{R^{2}-4}\right]^{1 / 2}-1$.

where

$R=B G+B Y-2 \alpha A$.
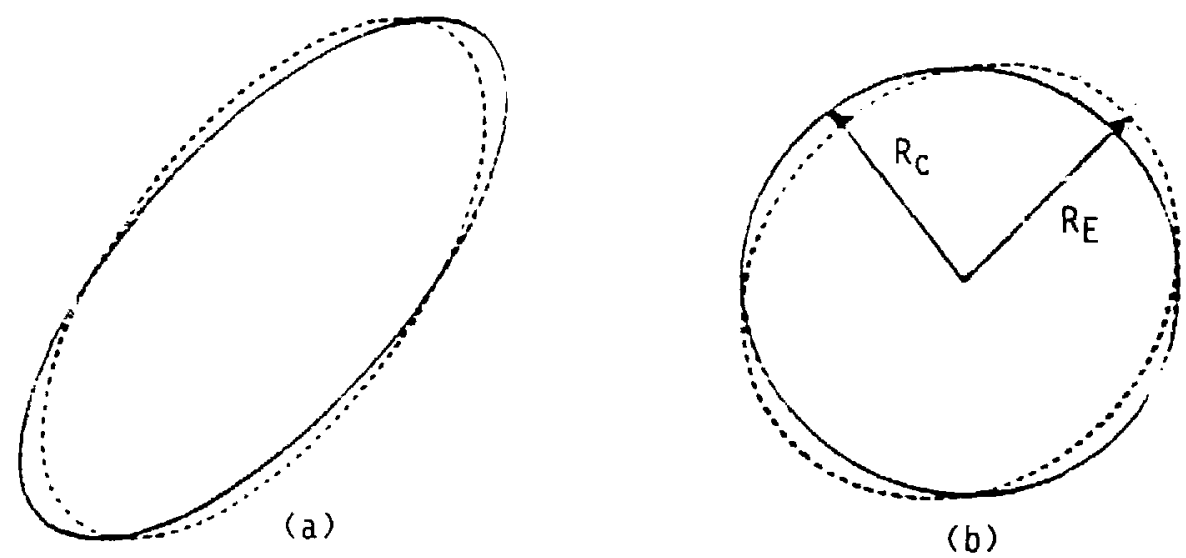

Fig. C-1. An example of two ellipes having a mismatch factor of

0.1 , shown before (a) and after (b) being transformed to a coordinate system in which the solid ellipse is a circle. 


\section{APPENDIX 0}

\section{RMS EMITTANCE AND THE EQUIVALENT UNIFORM BEAM}

The emittance in any phase plane is defined as the area enclosing all of the particle coordinates on that plane. In TRACE 3-D, the particles are assumed to be contained within a six-dimensional ellipsoid, and the emittance is the area of the ellipse that is the projection of the six-dimensional ellipsoid. The ellipse equation in the $x-x$ ' plane is

$y x^{2}+2 \alpha x x^{\prime}+\beta\left(x^{\prime}\right)^{2}=\varepsilon$.

where the parameters $\alpha, \beta, y$, and $\varepsilon$ are defined in App. $B$.

The second moments $\overline{x^{2}}, \overline{\left(x^{\prime}\right)^{2}}$, and $\overline{x x^{\prime}}$ can be calculated if the density of particles within the ellipse is known. Let $f\left(x, x^{\prime}\right)$ denote the particle density function in the $x-x^{\prime}$ plane, normalized so that

$$
\int f\left(x, x^{\prime}\right) d A=1 \text {. }
$$

where the integraticil is over the area of the ellipse. Then

$\overline{x^{2}}=\int x^{2} f\left(x, x^{\prime}\right) d A$.

For a uniform density on the $x-x^{\prime}$ plane, $f\left(x, x^{\prime}\right)$ is the reciprocal of the ellip:e area, and the second moments are easily calculated:

$$
\overline{x^{2}}=\frac{1}{\pi \varepsilon} \int_{-x_{m}}^{x_{m}} x^{2} h(x) d x
$$

where $x_{m}=\sqrt{B \varepsilon}$ is the maximum extent of the ellipse in the $x$-direction, and $h(x)$ is the width of the ellipse in $x^{\prime}$ at a given value of $x$,

$$
h(x)=\frac{2 \sqrt{B \varepsilon-x^{2}}}{\beta} .
$$


The results for the three second moments are

$\overline{x^{2}}=\beta \varepsilon / 4$,

$\overline{\left(x^{\prime}\right)^{2}}=\gamma \varepsilon / 4$

and

$\overline{x x^{\prime}}=-\alpha \varepsilon / 4$.

Because $\beta \gamma-\alpha^{2}=1$,

$\pi \sqrt{\overline{x^{2}} \overline{\left(x^{\prime}\right)^{2}}-\overline{x x^{\prime}}}=\pi \varepsilon / 4$.

The term on the left-hand side is defined as the rms emittance. The rms emittance can be calculated for any arbitrary distribution whose second moments can be measured or calculated. The "equivalent uniform beam" is defined to be the beam having a uniform charge distribution and having the same second moments as the arbitrary distribution. Notice that a longitudinally continuous uniform beam has a total emittance that is four times its rms emittance.

In TRACE 3-D, we assume linear space-charge forces, which implies a uniform charge distribution in an elipsoid in $x-y-z$ space. Let us assume that the distribution in any three-dimensional projection of the sixdimensional hyperellipsoid is uniform. When calculating second moments for the $x$ - and $x^{i}$-coordinates, take $z$ to be the third coordinate of the uniform ellipsoid and further assume that $z$ is uncorrelated with $x$ and $x$ '. The intersection of the ellipsoid with the $x-x$ ' plane is the ellipse defined by $y x^{2}+2 \alpha x x^{\prime}+B\left(x^{\prime}\right)^{2}=\varepsilon$.

At any $z$ between $-\hat{z}$ and $+\hat{z}$, where $\hat{z}$ is the extent of the ellipsoid in the $z$-direction, the intersection of the ellipsoid with a plane parallel to the $x-x^{\prime}$ plane is an ellipse defined by $y x^{2}+2 \alpha x x^{\prime}+B\left(x^{\prime}\right)^{2}=\varepsilon\left[1-(z / \hat{z})^{2}\right]$.

The area of the ellipse is reduced from that of the midplane ellipse by the factor $\left[1-(z / \hat{z})^{2}\right]$. Then the second moments of the ellipse in this plane 
are

$\overline{x^{2}(z)}=B \varepsilon\left[1-(z / \hat{z})^{2}\right] / 4$

$$
=\overline{x^{2}(0)}\left[1-(z / \hat{z})^{2}\right] \text {. }
$$

$\overline{\left(x^{\prime}\right)^{2}(z)}=\overline{\left(x^{\prime}\right)^{2}(0)}\left[1-(z / \hat{z})^{2}\right]$.

and

$\overline{x x^{\prime}(z)}=\overline{x x^{\prime}(0)}\left[1-(z / \hat{z})^{2}\right]$.

The second moments over the entire ellipsoid are obtained by integration over z:

$\overline{x^{2}}=\overline{x^{2}(0)} \int_{-\hat{z}}^{\hat{z}}\left[1-(z / \hat{z})^{2}\right] f(z) d z$.

But $f(z)$ is the area of the ellipse at $z$ divided by the volume of the ellipsoid:

$f(z)=\frac{3}{4 \hat{z}}\left[1-(z / \hat{z})^{2}\right]$.

Therefore

$$
\begin{aligned}
\overline{x^{2}} & =\overline{x^{2}(0)} \frac{3}{\overline{4} \bar{z}} \int_{-\hat{z}}^{\hat{z}}\left[1-(z / \hat{z})^{2}\right]^{2} d z \\
& =\frac{4}{5} \overline{x^{2}(0)} \\
& =B \varepsilon / 5 .
\end{aligned}
$$

Similarly,

$\overline{\left(x^{\prime}\right)^{2}}=y \varepsilon / 5$, 


$$
\begin{aligned}
& \overline{x^{\prime}}=-\alpha \varepsilon / 5, \\
& \text { and } \\
& \overline{x^{2}} \overline{\left(x^{\prime}\right)^{2}}-\overline{x x^{\prime}}=(\varepsilon / 5)^{2} .
\end{aligned}
$$

The equivalent uniform beam in three-dimensions has an emittance five times larger than the rms emittance. 


\section{APPENDIX E}

\section{FIELD FORMULAE FOR PERMANENT-MAGNET QUADRUPOLE (PMQ)}

The PMQ field formulae are obtained from Ref. 6, to which we refer the reader for further discussion and for the assumptions under which the formulae are valid. The formulae are based on the REC quadrupole of the standard design using trapezoidal blocks as shown in Fig. E-l and are derived using a semi-infinite model with a flat-cut end (Fig. E-2).

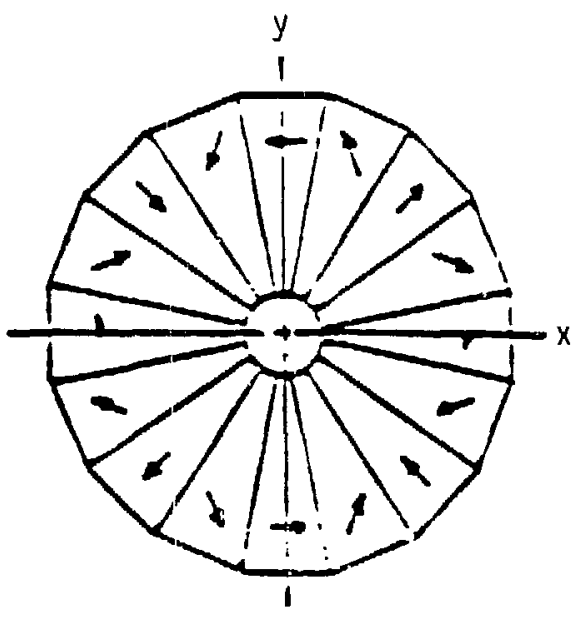

Fig. E-1. Cross section of segmented REC quadrupole (beam perpendicular to drawing plane).
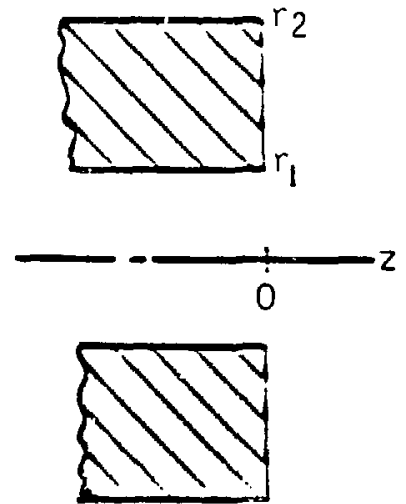

Fig. E-2. Cross section through semifinite REC quadrupole (beam in drawing plane).

The fringe field to first order is as follows:

$B_{x}(x, y, z)=G(z) y=G_{0} F(z) y$,

$B_{y}(x, y, z)=G(z) x=G_{0} F(z) x$, and

$\mathrm{B}_{\mathrm{Z}}=0$

where the gradient $G(z)$ is expressed in terms of the peak value $G_{0}$ times the fringe-field function $F(z)$ :

$G(z)=G_{0} F(z)$, 
and where the fringe field function $F(z)$, as shown in Fig. E-3, is given by $F(z)=\frac{1}{2}\left\{1-\frac{z}{8}\left(\frac{1}{r_{1}}+\frac{1}{r_{2}}\right)\left[\frac{v_{1}^{2} v_{2}^{2} v_{1}^{2}+v_{1} v_{2}+v_{2}^{2}+4+8 / v_{1} v_{2}}{v_{1}+v_{2}}\right]\right\}$,

where

$v_{i}=\left[1+\left(\frac{z}{r_{i}}\right)^{2}\right]^{-1 / 2}, \quad i=1,2, \ldots$

$\mathrm{G}_{0}=2 \mathrm{~B}_{r} \mathrm{C}_{2}\left(3 / r_{1}-1 / r_{2}\right)=2 \mathrm{G}(0)=\mathrm{G}(-\infty)$,

$B_{r}=$ magnitude of remnant magnetization of REC material,

$r_{1}=$ inner radius of quadrupole,

$r_{2}=$ outer radius of quadrupole,

$C_{2}=\sin (3 \pi / M) /(3 \pi / M)$, and

$M=$ number of trapezoidal blocks composing the magnet.

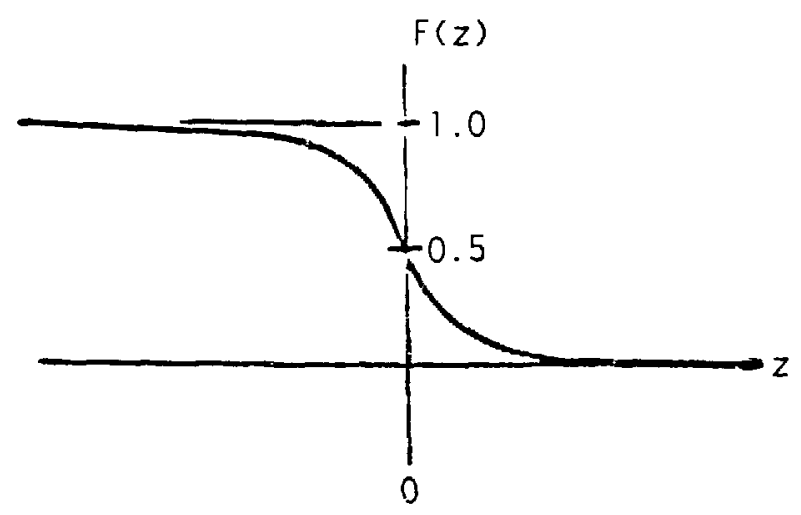

Fig. E-3. Quadrupole fringe-field function. Note that $F(-z)=1-F(z)$.

To find the fringe field for a quadrupole of finite physical length $\ell_{p}$, a second semi-infinite magnet of opposite sign is superimposed on the first semi-infinite magnet with a nonoverlapping distance equal to $l_{p}$. The fields are added, resulting in the fringe field as shown in the lower portion of Fig. E-4. 
For doublets, triplets, or any combination of PMQs, the total fringe field $G_{t}$ is found by summing the individual gradients $G(z)$ as follows:

$G_{t}=\sum_{i=1}^{n} G_{i}\left(z_{i}\right)=G_{0 i} F_{i}\left(z_{i}\right), \quad i=1, n$.

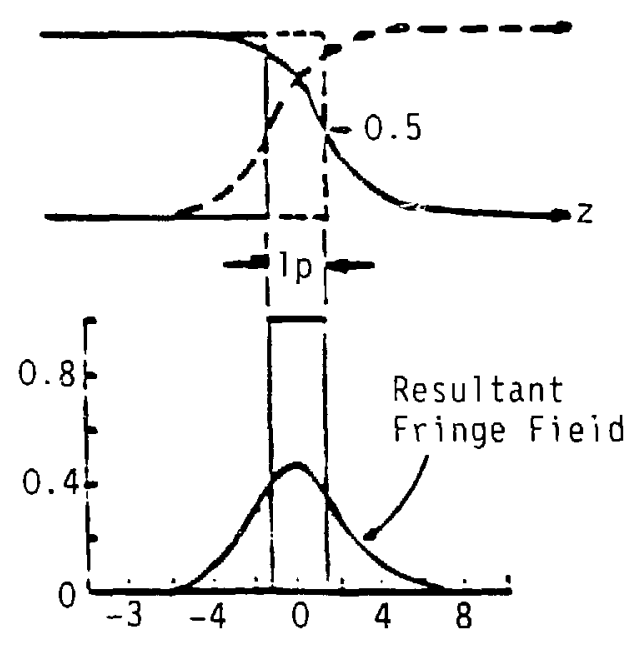

Fig. E-4. Two semi-infinite magnets, nonoverlapping by $\ell_{p}$, resulting in fringe field for $P M Q$ of length $\ell_{p}$. 
Weiss ${ }^{12}$ has calculated the increase in the rms emittance of a bunched beam as it crosses an $r f$ gap. The gap is treated as a thin lens, and the impulse coefficients are given in Sec. VI.J of this report. The emittances increase because the transverse impulses depend on $\phi$, the phase of the $r f$ when a particle arrives at the gap, and $\phi$ differs from one end of the bunch to the other. The transfer matrix is calculated assuming that the phase is constant at $\phi_{5}$, the phase when the beam center is at the gap.

At the gap, the $x$-coordinate is unchanged and the $x^{\prime}$-coordinate is changed by $x_{f}^{\prime}=n x_{i}^{\prime}+k_{x}^{\prime} \sin \phi x_{i}$,

where

$n=\left(B_{Y}\right)_{j} /\left(B_{Y}\right)_{f}$,

$k_{x}^{\prime}=\frac{-\pi|q| E_{o} T L}{m_{o} c^{2} \bar{\beta}^{2}-\bar{y}^{2}(B y)_{f} \lambda}$.

the $i$ and $f$ subscripts denote initial (before the gap) and final (after the gap) values, and the bars denote average values.

The second moments before and after the gap are related as follows:

$\overline{x_{f}^{2}}=\overline{x_{i}^{2}}$

$\overline{\left(x_{f}^{\prime}\right)^{2}}=n^{2} \overline{\left(x_{i}^{\prime}\right)^{2}}+2 n k_{x}^{\prime} \overline{x_{i} x_{i}^{\prime} \sin \phi}+\left(k_{x}^{\prime}\right)^{2} \overline{x_{i}^{2} \sin ^{2} \phi}$,

and

$\overline{x_{f} x_{f}^{\prime}}=n \overline{x_{i} x_{i}^{\prime}}+k_{x}^{\prime} \overline{x_{i}^{2} \sin \phi}$.

Assuming that $\phi$ is uncorrelated with $x$ and $x^{\prime}$, the averages are given by

$\overline{x_{i}^{2} \sin \phi}=\overline{x_{i}^{2}} \sin \phi_{S} f(\Delta \phi)$ 


$$
\overline{x_{i} x_{i}^{\prime} \sin \phi}=\overline{x_{i} x_{i}^{\prime}} \sin \phi_{s} f(\Delta \phi),
$$

and

$$
\overline{x_{i}^{2} \sin ^{2} \phi}=\overline{x_{i}^{2}} g\left(\phi_{s}, \Delta \phi\right)
$$

where $\Delta \phi$ is the half-width of the phase spread,

$$
f(\Delta \phi)=\frac{15}{(\Delta \phi)^{2}}\left[\frac{3}{(\Delta \phi)^{2}}\left(\frac{\sin \Delta \phi}{\Delta \phi}\right)-\cos \Delta \phi-\frac{\sin \Delta \phi}{\Delta \phi}\right]
$$

and

$g\left(\phi_{S}, \Delta \phi\right)=\frac{1}{2}\left[1+\left(\sin ^{2} \phi_{S}-\cos ^{2} \phi_{S}\right) f(2 \Delta \phi)\right]$.

In the limit, then $\Delta \phi \rightarrow 0, f(\Delta \phi) \rightarrow 1$ and $g\left(\phi_{\varsigma}, \Delta \phi\right)+\sin ^{2} \phi_{s}$.

Substituting the above averages into the equations for the second moments,

$\overline{\left(x_{f}^{\prime}\right)^{2}}=n^{2} \overline{x_{i}^{2}}+2 n k_{x}^{\prime} \sin \phi_{s} f(\Delta \phi) \overline{x_{i} x_{i}^{\prime}}+\left(k_{x}^{\prime}\right)^{2} g\left(\phi_{s}, \Delta \phi\right) \overline{x_{i}^{2}}$

and

$\overline{x_{f} x_{f}^{\prime}}=n \overline{x_{i} x_{i}^{\prime}}+k_{x}^{\prime} \sin \phi_{s} f(\Delta \phi) \overline{x_{i}^{2}}$.

If the original equation for $x_{f}$ is replaced by

$x_{f}^{\prime}=\eta x_{i}+k_{x}^{\prime} \sin \phi_{5} f(\Delta \phi) x_{i}$,

then one obtains the above second moments if a term $\overline{\Delta\left(x_{f}^{\prime}\right)^{2}}$ is added to $\overline{\left(x_{f}^{\prime}\right)^{2}}$, where

$$
\overline{\Delta\left(x_{f}^{\prime}\right)^{2}}=\left(k_{x}^{\prime}\right)^{2}\left[g\left(\phi_{s}, \Delta \phi\right)-\sin ^{2} \phi_{s} f^{2}(\Delta \phi)\right] x_{i}^{2} .
$$

The increase in the rms emittance is

$\Delta \varepsilon_{\text {rms }}=\sqrt{\overline{x_{f}^{2}} \overline{\Delta\left(x_{f}^{\prime}\right)^{2}}}$

73 


\section{APPENDIX G}

\section{ELECTROMAGNETIC FIELDS IN CYLINDRICAL CAVITIES 13,14}

For cylindrical cavities resonating in the $\mathrm{TM}_{010}$ standing-wave mode, the only nonzero components of the electromagnetic field are $E_{r}, E_{z}$, and ${ }_{\theta} \theta$, and these quantities are independent of $\theta$. The stationary solutions have a harmonic dependence on time: therefore,

$E_{z}(r, z, t)=E_{z}(r, z) \sin \omega t$,

where $w$ is the angular frequency of the standing wave. The term $E_{z}(r, z)$ can be expressed by a Fourier series in $z$. Let $L$ denote the length of the cavity, and take the origin of the coordinate system to be at the center of the cavity. Then

$E_{z}(r, z)=\sum_{m} \cdot\left[a_{m}(r) \cos \frac{m \pi z}{L}+b_{m}(r) \sin \frac{m \pi z}{L}\right]$.

We now restrict ourselves to consider accelerating structures operating in the $\pi$-mode in which alternating cavities are $180^{\circ}$ out of phase cone complete period consists of two cavities). We also assume $E_{z}$ to be symmetric about the center of the cavity. These restrictions imply that $b_{m}=0$ for all $m$, and $a_{m}=0$ when $m$ is zero and even integers. Then

$E_{z}(r, z, t)=\sum_{m} a_{m}(r) \cos \frac{m \pi z}{L} \sin \omega t, \quad m=1,3,5, \ldots,$.

Inserting this expression into the wave equation

$\frac{\partial^{2} E_{z}}{\partial z^{2}}+\frac{1}{r} \frac{\partial E_{z}}{\partial r}+\frac{\partial^{2} E_{z}}{\partial r^{2}}=\frac{1}{c^{2}} \frac{\partial^{2} E_{z}}{\partial t^{2}}$.

we obtain

$$
\left.\sum_{m} \frac{d^{2} a_{m}}{d r^{2}}+\frac{1}{r} \frac{d a_{m}}{d r}-k_{m}^{2} a_{m}\right]=0 \text {, }
$$

where 


$$
\begin{aligned}
k_{m}^{2} & =\left(\frac{m \pi}{L}\right)^{2}-\left(\frac{\omega}{c}\right)^{2} \\
& =\left(\frac{m \pi}{L}\right)^{2}-\left(\frac{2 \pi}{\lambda}\right)^{2} .
\end{aligned}
$$

Each term in the above sum will be zero if

$$
a_{m}=A_{m} I_{0}\left(k_{m} r\right) \quad \text { when } k_{m}^{2} \geq 0
$$

and

$a_{m}=A_{m} J_{0}\left(k_{m} r\right) \quad$ when $k_{m}^{2}<0$.

where $J_{0}$ is the standard Bessel function of order zero, and $I_{0}$ is the modified Bessel function of order zero. The value of $k_{m}^{2}$ will normally be positive (although if $L>\lambda / 2, k_{1}^{2}$ will be negative), thus the modified Bessel functions will be used below.

Having obtained an expression for $E_{z}(r, z, t)$, similar expressions can be obtained for $E_{r}(r, z, t)$ and $B_{\theta}(r, z, t)$ by using the Maxwell equations

$$
\nabla \cdot \vec{E}=0
$$

and

$\nabla \cdot \vec{B}=\frac{l}{c^{2}} \frac{\partial \vec{E}}{\partial t}$.

For our particular geometry and symmetry assumptions, these equations reduce to

$\frac{1}{r} \frac{\partial}{\partial r}\left(r E_{r}\right)=-\frac{\partial E_{z}}{\partial z}$,

$\frac{\partial B_{\theta}}{\partial z}=-\frac{1}{c^{2}} \frac{\partial E_{r}}{\partial t}$,

and

$\frac{1}{r} \frac{\partial}{\partial r}\left(r B_{\theta}\right)=\frac{1}{c} \frac{\partial E_{z}}{\partial t}$

The results, which can be verified by using the recurrence formula, 
$I_{i}(x)+x \frac{d}{d x} I_{1}(x)=x I_{0}(x)$,

are

$E_{z}(r, z, t)=\sum_{m} A_{m} I_{0}\left(k_{m} r\right) \cos \frac{m \| \pi z}{L} \sin \omega t$,

$E_{r}(r, z, t)=\sum_{m} \frac{A_{m} m \pi}{k_{m} L} I_{l}\left(k_{m} r\right) \sin \frac{m \pi z}{L} \sin \omega t$,

and

$B_{\theta}(r, z, t)=\sum_{m} \frac{A_{m}^{\omega}}{k_{m} c^{2}} I_{1}\left(k_{m} r\right) \cos \frac{m \pi z}{L} \cos \omega t$.

The $A_{m}$ coefficients can be determined for a particular geometry by Fourier analyzing the $E_{z}(r, z)$ values calculated by SUPERFISH. The results are usually normalized so that the average axial accelerating field $E_{0}$ is $1 \mathrm{MV} / \mathrm{m}$, where

$E_{0}=\frac{1}{L} \int_{-L / 2}^{L / 2} E_{z}(0, z) d z$

The field components are linearized by replacing $I_{0}\left(k_{m} r\right)$ by 1 and $I_{l}\left(k_{m} r\right)$ by $k_{m} r / 2$. Also, substituting $2 \pi c / \lambda$ for $\omega$ and $\phi$ for $\omega t$, the linearized components are

$E_{z}(r, z, \phi)=E_{0} \sum_{m} A_{m} \cos \frac{m \pi z}{L} \sin \phi$,

$E_{r}(r, z, \phi)=E_{0} \sum_{m} A_{m} \frac{m \pi}{2 L} \sin \frac{m \pi z}{L} \sin \phi r$,

and

$B_{\theta}(r, z, \phi)=E_{0} \sum_{m} A_{m} \frac{\pi}{c \lambda} \cos \frac{m \pi z}{L} \cos \phi r$.

In moving a distance $\Delta s$ through a constant accelerating field $E_{Z}$, the energy of the beam changes from $W$ to $W+\Delta W$, where 
$\Delta W=E_{Z} \Delta S$

The average energy over the distance $\Delta s$ is

$\bar{W}=W+\Delta W / 2$.

The change in the $x$-component of the normalized momentum that is due to constant $E_{r}$ and $B_{\theta}$ fields applied for a distance $\Delta s$ is

$$
\begin{aligned}
\Delta(B y)_{x} & =\frac{q \Delta s}{m_{0} c^{2} \bar{B}}\left(E_{r}-v_{z} B_{\theta}\right) x / r \\
& =\frac{\pi q E_{0} \Delta s}{m_{0} c^{2} \bar{B}} \sum_{m} A_{m}\left[\frac{m}{2 L} \sin \frac{m \pi z}{L} \sin \phi-\frac{\bar{B}}{\lambda} \cos \frac{m \pi z}{L} \cos \phi\right] x \\
& =k_{x} x .
\end{aligned}
$$

where $\bar{B}$ is v/c for particles having energy $\bar{W}$. The impulse coefficient $k_{y}$ is identical to $k_{x}$.

The change in $\Delta(B y)_{z}$ caused by a particle arriving at a particular location in the cavity when the rf phase is $\phi+\Delta \phi$ rather than $\phi$ is

$\Delta[\Delta(B y) z]=\frac{q \Delta s}{m_{0} c^{2} \bar{B}} \frac{\partial E_{z}}{\partial \phi} \Delta \phi$.

The longitudinal displacement, $z^{\prime}$, of a particle from the center of the beam is related to the phase displacement $\Delta \phi$ by

$z^{\prime}=-\beta \lambda \Delta \phi / 2 \pi$

so the change in $\Delta(B y)_{z}$ is

$$
\begin{aligned}
\Delta\left[\Delta(B y)_{z}\right] & =k_{z} z^{\prime} \\
& =\frac{-2 \pi q \Delta s E_{0}}{m_{0} c^{2} \bar{B}^{2} \lambda} \sum_{m} A_{m} \cos \frac{m \pi z}{L} \cos \phi z^{\prime} .
\end{aligned}
$$

In this appendix, $z$ denotes the longitudinal displacement from the center of the cavity, and $z^{\prime}$ denotes longitudinal displacement from the center of the beam ellipsoid. 


\section{APPENCIX H}

\section{COUPLEU-CAVITY TANKS}

A tank element implies a sequence of identical cylindrical cavities, coupled in some way to permit power flow. Tha fields in adjacent cells (cavities) are assumed to be $180^{\circ}$ out of phase, and a standing-wave $\mathrm{TM}_{0} 0$ mode is assumed. Tank transformations are valid when the beam velocity does not change appreciabily as the particles go through the tank, and the time-offlight through two cavities is approximately one rf period. In this case, the detailed shapes of the fields in a cavity are rot very important as long as their averages over one cavity (or a half-cavity) are correct.

A reasonable approxination for the longitudinal electric field is $E_{z}(r, z, t)=A I_{0}(k r) \cos \frac{\pi z}{L} \sin \omega t$

where

$k^{2}=\left(\frac{\pi}{L}\right)^{2}-\left(\frac{2 \pi}{\lambda}\right)^{2}$.

This is the first term in the Fourier expansion for $E_{z}(r, z, t)$ given in App. G. A quantity whose value is usually specified for a tank is $E_{0} T$, the effective accelerating field, the product of the average axial electric field, and the transit-time factor. This quantity is defined by

$E_{0} T=\frac{1}{L} \int_{-L / 2}^{L / 2} E_{z}(0, z) \cos (\pi z / L) d z$.

Putting in the assumed form for $E_{z}$ and integrating, we find

$$
A=2 E_{0}{ }^{\top}
$$

The three nonzero field components are

$E_{z}(r, z, t)=2 E_{0} I_{0}(k r) \cos (\pi z / L) \sin \omega t$,

$E_{r}(r, z, t)=\frac{2 \pi E_{0}{ }^{T}}{k L} I_{l}(k r) \sin (\pi z / L) \sin \omega t$, 
and

$B_{\theta}(r, z, t)=\frac{2 E_{0} T \omega}{k c^{2}} I_{l}(k r) \cos (\pi z / L) \cos \omega t$,

where $\omega=2 \pi c / \lambda$ is the angular frequency of the $r f$ and $z=\dot{u}$ at the middle of a cavity. Using the assumptions mentioned earlier, wt can be replaced by $\pi z / L+\phi+\pi / 2$, where $\phi$ is the phase of the $r f$ when the center of the beam arrives at the beginning of the cavity. Then

$\cos \omega t=\cos \pi z / L \cos (\phi+\pi / 2)-\sin \pi z / L \sin (\phi+\pi / 2)$

$=-\cos \pi z / L \sin \phi-\sin \pi z / L \cos \phi$,

$\sin \omega t=\sin \pi z / L \cos (\phi+\pi / 2)+\cos \pi z / L \sin (\phi+\pi / 2)$

$$
=-\sin \pi z / L \sin \phi+\cos \pi z / L \cos \phi .
$$

The calculation of the averages of the field components over each half of a cavity (assuming $r$ is constant) involves finding the averages of $\cos ^{2} \pi z / L$, $\sin ^{2} \pi z / L$, and sir. $\pi z / L \cos \pi \ddot{z} / L$. Over the first half of a cavity $(-L / 2 \leq z \leq 0)$,

$\overline{\cos ^{2} \pi z / L}=\overline{\sin ^{2} \pi z / L}=1 / 2$,

$\sin \pi z / L \cos \pi z / L=-1 / \pi$.

The average, linearized, field components in the first half of a cavity are

$\overline{E_{z 1}}=2 E_{0}{ }^{\top}\left(\frac{\sin \phi}{\pi}+\frac{\cos \phi}{2}\right)$,

$\overline{E_{r 1}}=-\frac{\pi E_{0}{ }^{\top}}{L}\left(\frac{\cos \phi}{\pi}+\frac{\sin \phi}{2}\right) r$,

$\overline{B_{\theta l}}=\frac{\pi E_{0} T B}{c L}\left(\frac{\cos \phi}{\pi}-\frac{\sin \phi}{2}\right) r$,

where we have used $L=\beta \lambda / 2$. The change in the beam energy in the first haif of a cavity is 
$\Delta W_{1}=q \overline{E_{21}} L / 2$

$$
=q E_{0} T L\left(\frac{\cos \phi}{2}+\frac{\sin \phi}{\pi}\right) \text {. }
$$

The average energy in the first half is assumed to be

$\overline{W_{1}}=W+\Delta W_{1} / 2$,

where $W$ is the energy at the beginning of the cavity. The average normalized velocity $\overline{B_{1}}$ is calculated from $\overline{W_{1}}$. The average radial force is

$\overline{F_{r 1}}=q\left(\overline{E_{r l}}-\overline{\beta_{1} c} \overline{B_{\theta l}}\right)$.

so the change in the $x$-component of the normalized momentum is

$\Delta(B Y)_{X}=k_{X} 1^{x}$

$$
=-\frac{\pi q E_{0} T}{2 m_{0} c^{2} \bar{\beta}_{1}}\left[\left(1+{\overline{\beta_{1}}}^{2}\right) \frac{\cos \phi}{\pi}+\left(1-{\overline{\beta_{1}}}^{2}\right) \frac{\sin \phi}{2}\right] x .
$$

The vertical impulse coefficient $k_{y l}$ is the same as $k_{x l}$. In the above expression, $\overline{B_{1}}$ should actually be $\overline{\beta_{1}} \beta$, where $\beta=2 L / \lambda$, but the assumptions imply that $\bar{B}_{1} \approx \beta$.

The change in $\Delta(B y)_{z}$ caused by a particle having a displacement $z^{\prime}$ from the center of the beam is

$$
\begin{aligned}
\Delta\left[\Delta(B \gamma)_{z}\right] & =k_{z l^{\prime}} z^{\prime} \\
& =\frac{q L}{2 m_{0} c^{2} \bar{\beta}_{1}} \frac{\partial \overline{E_{z l}}}{\partial \phi} \frac{\partial \phi}{\partial z^{\prime}} z^{\prime} \\
& =\frac{q \pi E_{0}{ }^{\top}}{m_{0} c^{2} \bar{\beta}_{1}}\left(\frac{\sin \phi}{2}-\frac{\cos \phi}{\pi} z^{\prime}\right),
\end{aligned}
$$

where

$$
Z^{\prime}=-\frac{\pi}{L} \Delta \phi
$$


In the second half of a cavity, the averages of the field components are

$\overline{E_{z 2}}=2 E_{0} T\left(\frac{\cos \phi}{2}-\frac{\sin \phi}{\pi}\right)$.

$\overline{E_{r 2}}=\frac{\pi E_{0}{ }^{\top}}{L}\left(\frac{\cos \phi}{\pi}-\frac{\sin \phi}{2}\right) r$.

$\overline{B_{\theta 2}}=-\frac{\pi E_{O} T B}{C L}\left(\frac{\cos \phi}{\pi}+\frac{\sin \phi}{2}\right) r$.

The energy charge in the second half is

$\Delta W_{2}=q E_{O} T L\left(\frac{\cos \phi}{2}-\frac{\sin \phi}{\pi}\right)$,

and the average energy $\overline{W_{2}}$ and the corresponding $\overline{B_{2}}$ can be calculated. The impulse coefficients for the second half are

$k_{x 2}=-\frac{\pi q E_{0}{ }^{\top}}{2 m_{0} c^{2} \overline{\beta_{2}}}\left[\left(1-{\overline{\beta_{2}}}^{2}\right) \frac{\sin \phi}{2}-\left(1+{\overline{\beta_{2}}}^{2}\right) \frac{\cos \phi}{\pi}\right]$,

$k_{y 2}=k_{x_{2}}$,

$k_{z 2}=\frac{\pi q E_{0} T}{m_{0} c^{2} \overline{B_{2}}}\left(\frac{\sin \phi}{2}+\frac{\cos \phi}{\pi}\right)$ 


\section{APPENDIX I \\ TRANSVERSE EMITTANCE INCREASE FROM CHROMATIC ABERRATIONS}

The focusing and defocusing forces actirig on a particle passing through a magnetic quadrupole depend on the particle's momentum $p$. An energy spread can, therefore, cause an increase in the effective transverse emittance. This increase can be calculated if we approximate a quadrupole by a sequence of drifts and thin lenses. At each lens, the $x$ '-coordinate of a particle having a momentum $p+\Delta p$ is modified by

$x_{f}^{\prime}=x_{i}^{1}-k_{x} x_{i} /(1+\delta)$,

where

$\delta=\Delta p / p$,

$k_{x}=q B^{\prime} \Delta s / m_{0} C B Y$.

and the $i$ and $f$ subscripts denote initial and final values. The quantity $k_{x}$ is the impulse coefficient for a thin-lens equivalent for a quadrupole having a magnetic gradient $B^{\prime}$ and effective length $\Delta s$.

The second moments of the initial and final coordinates are related by

$\overline{x_{f}^{2}}=\overline{x_{i}^{2}}$,

$\overline{\left(x_{f}^{\prime}\right)^{2}}=\overline{\left(x_{j}^{\prime}\right)^{2}}-2 k_{x} \overline{x_{j} x_{i}^{1} /(1+\delta)}+k_{x}^{2} \overline{x_{i}^{2}(1+\delta)^{2}}$,

and

$\overline{x_{f} x_{f}^{1}}=\overline{x_{j} x_{j}^{1}}-k_{x} \overline{x_{j}^{2} /(1+\delta)}$.

The averages are calculated over the volume occupied by the hyperellipsoid representing the beam. Let $\overline{x_{i} x_{j}^{\prime}}(\delta)$ denote the average of $x_{i} x_{i}^{\prime}$ at a particular $\delta$. Then

$\overline{x_{i} x_{i}^{\prime}}=\int_{-\delta}^{\hat{\delta}} \overline{x_{i} x_{i}^{\prime}}(\delta) f(\delta) d \delta$,

where $\delta$ is the maximum value of $\delta$ in the hyperellipsoid, and $f(\delta)$ is the density function that satisfies

82 
$\int_{-\hat{\delta}}^{\hat{\delta}} f(\delta) d \delta=1$.

If we assume a uniform ellipsoid in the $x, x^{\prime}$, $\delta$ space, then

$f(\delta)=\frac{3}{4 \delta}\left[1-(\delta / \delta)^{2}\right]$.

Also, if we assume $x$ and $x^{\prime}$ are uncorrelated with $\delta$, then the effect of a nonzero value of $\delta$ is to reduce the space available to $x$ and $x^{\prime}$ :

$\overline{x_{i} x_{i}^{!}}(\delta)=\left[1-(\delta / \hat{\delta})^{2}\right] \overline{x_{i} x_{i}^{\prime}}(0)$.

Then

$\overline{x_{i} x_{i}^{1}}=\overline{x_{i} x_{i}^{1}}(0) \frac{3}{4 \hat{\delta}} \int_{-\hat{\delta}}^{\delta}\left[1-(\delta / \hat{\delta})^{2}\right]^{2} d \delta$

$=\frac{4}{5} \overline{x_{i} \times}(0)$

and

$$
\begin{aligned}
\overline{x_{i} x_{i}^{1} /(1+\delta)} & =\overline{x_{i} x_{i}^{1}}(0) \frac{3}{4 \hat{\delta}} \int_{-\delta}^{\hat{\delta}} \frac{\left[1+(\delta / \hat{\delta})^{2}\right]^{2}}{1+\delta} d \delta \\
& =\overline{x_{i} x_{i}^{1}}(0)\left(\frac{12}{15}+\frac{4}{35} \hat{\delta}^{2}\right) \\
& =\overline{x_{i} x_{i}^{1}}\left(1+\hat{\delta}^{2} / 7\right) .
\end{aligned}
$$

Repeating this procedure for calculating the other two averages, we find

$\overline{x_{i}^{2} /(1+\delta)}=\overline{x_{i}^{2}}\left(1+\delta^{2} / 7\right)$

and

$\overline{x_{i}^{2} /(1+\delta)^{2}}=\overline{x_{i}^{2}}\left(1+3 \delta^{2} / 7\right)$. 
The second moments after the thin lens are

$x_{f}^{2}=\overline{x_{i}^{2}}$

$\overline{\left(x_{f}^{\prime}\right)^{2}}=\overline{\left(\bar{x}_{i}^{\prime}\right)^{2}}-2 k_{x} \overline{x_{i} x_{i}^{\prime}}\left(1+\hat{\delta}^{2} / 7\right)+k_{x}^{2} \overline{x_{i}^{2}}\left(1+\frac{3}{7} \hat{\delta}^{2}\right)$,

$\overline{x_{f} x_{f}^{\prime}}=\overline{x_{i} x_{i}^{1}}-k_{x} \overline{x_{i}^{2}}\left(1+\hat{\delta}^{2} / 7\right)$.

If the original equation for $x_{f}^{\prime}$ is replaced by

$$
x_{f}^{\prime}=x_{i}^{1}-k_{x}\left(1+\delta^{2} / 7\right) x_{i} .
$$

then the above second moments are obtained if a term $\overline{\Delta\left(x_{f}^{\prime}\right)^{2}}$ is added to $\overline{\left(x_{f}^{\prime}\right)^{2}}$, where

$$
\overline{\Delta\left(x_{f}^{1}\right)^{2}}=k_{x}^{2} \overline{x_{i}^{2}}\left[\left(1+\frac{3}{7} \hat{\delta}^{2}\right)-\left(1+\hat{\delta}^{2} / 7\right)^{2}\right]_{i} \text {. }
$$

The increase in the rms remittance is

$$
\begin{aligned}
\Delta \varepsilon_{\mathrm{rms}} & =\sqrt{\overline{x_{f}^{2}} \overline{\Delta\left(x_{f}^{\prime}\right)^{2}}} \\
& \approx \overline{x_{i}^{2}} k_{x} \hat{\delta} / \sqrt{7} .
\end{aligned}
$$

84 


\section{APPENDIX J \\ CALCULATION OF TRANSVERSE EMITTANCES \\ FOR BEAM-WIDTH MEASUREMENTS AT THREE LOCATIONS}

If the width of the beam is measured at three separate locations, and if the transfer matrices between these locations are known, then the transverse emittances and ellipse parameters can be calculated. Let $\sigma^{\mathrm{m}}$ denote the o-matrix at the mth measurement iocation, where m is 1,2 , or 3 . The measurements define the 1,1 and 3,3 elements at each location. The unknown quantities are $\sigma_{12}, \sigma_{22}, \sigma_{34}$, and $\sigma_{44}$ at each location. But if these quantities are known at any of the three locations, they can be calculated at the other two locations using the assumed known transfer matrices.

Let $\sigma^{\prime}$ denote the initial estimate of the o-matrix at the first measurement location, and $\delta$ denote the (unknown) correction matrix for $\sigma{ }^{1}$. That is, the actual o-matrix at the first measurement is $\sigma l+\delta$. Because $\sigma_{11}^{1}$ and $\sigma_{33}^{1}$ are given by the measurement, the only nonzero elements of the $\delta$-matrix are $\delta_{12}\left(=\delta_{21}\right), \delta_{22}, \delta_{34}\left(=\delta_{43}\right)$, and $\delta_{44}$. Let $R$ denote the transfer matrix from the first to the second measurement location, and $r_{i j}$ be the $i, j$ th element of this matrix. Then

$\sigma^{2}=R \sigma^{\prime} R^{\top}+R \delta R^{\top}$.

From measurements at the second iocation, $\sigma_{11}^{2}$ and $\sigma_{33}^{2}$ are known, giving two equations to be satisfied by the elements of the $\delta$-matrix:

$\left[\sigma^{2}-R_{\sigma} l_{R}^{\top}\right]_{11}=2 r_{11} r_{22} \delta_{12}+r_{12}^{2} \delta_{22}+2 r_{13} r_{14} \delta_{34}+r_{14}^{2} \delta_{44}$,

and

$\left[\sigma^{2}-R_{0}{ }^{l} R^{\top}\right]_{33}=2 r_{31} r_{32} \delta_{12}+r_{32}^{2} \delta_{22}+2 r_{33} r_{34} \delta_{34}+r_{34}^{2} \delta_{44}$.

Two similar equations exist for the beam measurements at the third location in terms of the $\sigma^{1}$ - and $\delta$-matrix elements and the transfer matrix between the first and third locations. The elements of the $\delta$-matrix can be determined from these four linear equations.

When space-charge forces are involved, an iterative procedure must be used because the transfer matrices depend on the beam profiles. In TRACE 3-D, 
the zero-current solution is used as the first step in the iteration. Successive steps use the previously determined $\sigma^{l}$-matrix as the input beam to be followed through the transport system to the measurement locations.

\section{REFERENCES}

1. K. L. Brown and S. K. HOWry, "TRANSPORT/360," Stanford Linear Accelerator Center report 91 (1970).

2. K. R. Crandall, "TRACE: An Interactive Beam-Transport Program," Los Alamos Scientific Laboratory report LA-5332 (October 1973).

3. K. R. Crandal1, "TRACE: An Interactive Beam-Transport Program for Unbunched Beams, and "Addendum to Program TRACE." CERN/PS/LIN/Note 77-3, Geneva, Switzerland (February 1977).

4. K. R. Crandall and D. P. Rusthoi, "Documentation for TRACE: An Interactive Beam-Transport Code," Los Alamos National Laboratory report LA-10235-MS (January 1985).

5. F. Sacherer, "RMS Envelope Equation with Space Charge," CERN/SI/Internal report 70-12, Geneva, Switzerland (1970).

6. K. Halbach, "Hhysical and Optical Properties of REC Magnets," Nuc 1. Instrum and Methods 187 (1981), 109-117.

7. A. P. Banford, The Transport of Charged Particle Beams, 〈E. \& F. N. Spon Ltd., London, 1966).

8. K. L. Brown, "A First-and Second-Order Matrix Theory for the Design of Beam Transport Systems and Charged Particle Spectrometers," Stanford Linear Accelerator Center report 75 (1969).

9. K. Halbach and R. F. Holsinger, "SUPERFISH - A Computer Program for Evaluation of rf-Cavities with Cylindrical Symmetry, "Particle Accelerators, Vol. $7(1976), 213-222$.

10. P. M. Lapostolle, CERN report AR/:- SG/65-15, Geneva, Switzerland (July 1965).

11. R. S. Mills, K. R. Crandall, and J. A. Farrell, "Designing Self-Matching Linacs," Proc. 1984 Linac Conf., Gesellschaft für Schwerionenforschung, May 7-11, 984, Darmstadt report GSI-84-11, 112 (September 1984).

12. M. Weiss, "Bunching of Intense Proton Beams with Six-Dimensional Matching to the Linac Acceptance," CERN/MPS/LI report 73-2 Geneva, Switzerland (1978).

13. H. G. Hereward, "The General Theory of Linear Accelerators, " in Linear Accelerators, F. N. Lapostolle and A. L. Septier, Eds. (North Holland Publishing Co. , Amsterdam, 1969).

14. L. Smith, "Linear Accelerators," in Hanbuch der Physik, (Springer Verlag, Berlin, 1959), Vol. 44. 